\title{
Exploring the formation histories of galaxies - globular clusters and beyond
}

\author{
Dissertation \\ zur Erlangung des Doktorgrades \\ der Mathematisch-Naturwissenschaftlichen Fakultäten \\ der Georg-August-Universität zu Göttingen
}

vorgelegt von

Thomas Lilly

aus Bonn

Göttingen 2007 
D 7

Referentin: Prof. Dr. U. Fritze - v. Alvensleben Korreferent: Prof. Dr. Wolfram Kollatschny

Tag der mündlichen Prüfung: 


\section{Contents}

1 Introduction \& Overview 1

2 Tracing back the star formation histories of galaxies 3

2.1 Introduction . . . . . . . . . . . . . . . 4

2.2 Models and input physics . . . . . . . . . . . . . . 6

2.3 Scenarios in integrated light . . . . . . . . . . . . . . 7

2.3.1 Colours ................... 8

2.3 .2 Lick indices . . . . . . . . . . . . . . . . . . . . . . . . . . . 13

2.3 .3 Spectra ...................... 16

2.3.4 Some remarks on CMDs and integrated light . . . . . . . 18

2.4 The LMC bar field and its spectrum . . . . . . . . . . . . . . . 21

2.4.1 A simple 3-phase SFH . . . . . . . . . . . . . . . 22

2.4.2 Some experimentation ..................... 23

2.4.3 The CMD based SFH ............. . . 25

2.4.4 CMDs and integrated light: Comparison and Conclusions 28

2.5 Summary and Conclusions . . . . . . . . . . . . . . . . . 29

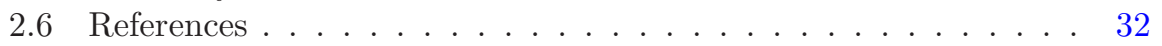

3 Analysing globular cluster observations I 35

3.1 Introduction . . . . . . . . . . . . . . . . . 36

3.2 Models and input physics . . . . . . . . . . . . . . . . 37

3.2.1 Evolutionary synthesis of Lick indices . . . . . . . . . . . 37

3.2.2 Non-solar abundance ratios . . . . . . . . . . . . . . 39

3.2.3 SSP model indices: Some examples . . . . . . . . . . . 40

3.3 Index sensitivities . . . . . . . . . . . . . . . . . . . . . . . . . . . . . . . 43

3.4 The Lick index analysis tool . . . . . . . . . . . . . . . . . . . . . 47

3.4.1 The $\chi^{2}-$ approach . . . . . . . . . . . . . . . 47

3.4.2 Examples and tests I: Galactic GCs . . . . . . . . . . . 47

3.4.3 Examples and tests II: M31 GCs and non-solar abundance ratios ........................ 53

3.5 Summary and outlook . . . . . . . . . . . . . . 56

3.6 References ....................... 57

4 Analysing globular cluster observations II $\quad 59$

4.1 Introduction . . . . . . . . . . . . . . . . 59

4.2 Models . . . . . . . . . . . . . . . . . 60

4.3 Analysis tools . . . . . . . . . . . . . . . . . . . 60

4.3.1 AnalySED and Lick Analysis Tool . . . . . . . . . . . 60 


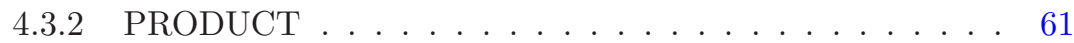

4.4 Examples and tests . . . . . . . . . . . . . . . . 62

4.4 LMC . . . . . . . . . . . . . . . . . 62

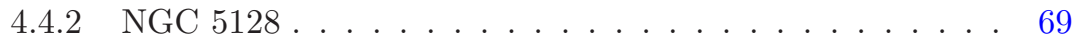

4.5 Intermediate-age GC populations and colour-metallicity-relations 73

4.6 Summary/Conclusions . . . . . . . . . . . . . . . . . . . 74

4.7 References . . . . . . . . . . . . . . . . . 75

4.8 Appendix .......................... 76

5 Applications to the globular cluster system of NGC $5128 \quad 81$

5.1 Introduction . . . . . . . . . . . . . . 81

5.2 Cluster sample and data analysis . . . . . . . . . . . . . . . . . . . . . 82

5.3 Results and discussion . . . . . . . . . . . . . . . . . . . . . 84

5.3.1 Age and metallicity distributions . . . . . . . . . . . 84

5.3.2 Correlations with galactic position . . . . . . . . . 87

5.4 Summary . . . . . . . . . . . . . . . . . . . 87

5.5 References..................... 87

$\begin{array}{llr}6 & \text { Summary } & 89\end{array}$

7 Acknowledgements $\quad 93$ 


\section{List of Figures}

2.1 Confrontation of the photometric evolution of scenarios $0,2,4$, 6 (left) and $0 \mathrm{~b}, 2 \mathrm{~b}, 4 \mathrm{~b}, 6 \mathrm{~b}$ (right), respectively, in terms of the colours $\mathrm{U}-\mathrm{V}, \mathrm{V}-\mathrm{K}($ top $)$, and $\mathrm{B}-\mathrm{V}, \mathrm{V}-\mathrm{I}$ (bottom), respectively. At 6.4 Gyr, when the latest phase of enhanced SF ends, a thick line is plotted. The bottom plots show the respective SFHs to be compared. . . . . . . . . . . . . .

2.2 (Left:) Photometric evolution in $\mathrm{V}-\mathrm{K}$ of a 'realistic' starburst (solid line) as compared to a rectangular one (dotted line). (Right:) Respective SFHs. See text for details. . . . . . . . . . . . . . . 10

2.3 Confrontation of the photometric evolution of scenarios 1, 3, 4 (left) and $1 \mathrm{~b}, 3 \mathrm{~b}, 4 \mathrm{~b}$ (right), respectively, in terms of the colours $\mathrm{U}-\mathrm{V}, \mathrm{V}-\mathrm{K}(t o p)$, and $\mathrm{B}-\mathrm{V}, \mathrm{V}-\mathrm{I}$ (bottom), respectively. At 6.4 Gyr, when the latest phase of enhanced SF ends, a thick line is plotted. The bottom plots show the respective SFHs to be compared. . . . . . . . . . . . . . .

2.4 Confrontation of the evolution of scenarios 0, 2, 4, 6 (left) and 0b, $2 \mathrm{~b}, 4 \mathrm{~b}, 6 \mathrm{~b}$ (right), respectively, in terms of the Lick indices $\mathrm{H} \beta$ and Fe5335. At $6.4 \mathrm{Gyr}$, when the latest phase of enhanced SF ends, a thick line is plotted. The bottom plots show the respective SFHs to be compared. . . . . . . . . . . . . 13

2.5 Confrontation of the evolution of scenarios 1, 3, 4 (left) and 1b, $3 \mathrm{~b}, 4 \mathrm{~b}$ (right), respectively, in terms of the Lick indices $\mathrm{H} \beta$ and Fe5335. At 6.4 Gyr, when the latest phase of enhanced SF ends, a thick line is plotted. The bottom plots show the respective SFHs to be compared. . . . . . . . . . . . . .

2.6 Top: Absolute differences of all 25 Lick indices (in terms of index strength) of the scenarios 0 and 3 and of the scenarios 4 and 3 , respectively, for the four model ages indicated in the plot. Bottom: SFHs of the scenarios; the ages 6.3, 6.6, 7.0, 7.7 and 10 Gyr are indicated by dashed lines. . . . . . . . . . . .

2.7 Top: Relative differences of the spectra of the scenarios 0 and 3 (left) and of the scenarios 4 and 3 (right) within the range of the Balmer lines (with markings of the Balmer lines $H_{\alpha}-H_{\eta}$ ) for the five model ages indicated in the plot. Bottom: $\mathrm{SFH}$ of the respective scenarios; the ages $6.3,6.6,7.0,7.7$ and 10 Gyr are indicated by dashed lines. . . . . . . . . . . . . . . 
2.8 Comparision of CMDs in $\mathrm{U}-\mathrm{V}, \mathrm{V}-\mathrm{I}$, and $\mathrm{V}-\mathrm{K}$ for three pairs of SSP models with different age metallicity combinations: Two young SSPs (a), two old SSPs (b), and an old and an intermediate age $\operatorname{SSP}(\mathrm{c}) \ldots \ldots \ldots \ldots \ldots \ldots \ldots \ldots$

2.9 Integrated-light spectrum of the LMC bar field (dereddened). Left: Original spectrum. Right: Spectrum with lowered resolution to be compared with model spectra, and the three filters used for the analysis. . . . . . . . . . . . . . . 21

2.10 Photometric evolution of a simple 3-phase scenario in terms of $(\mathrm{B}-\mathrm{V})_{H S T},(\mathrm{~V}-\mathrm{R})_{H S T}$, and $(\mathrm{B}-\mathrm{R})_{H S T}$ with the corresponding $\mathrm{SFH}$; the observed LMC bar field colours (cf. Table 2.3) are marked with black dots at 15 Gyr galaxy age. . . . . . . . . . .

2.11 Model spectrum of the 3-phase scenario (cf. Fig. 2.10) at a galaxy age of 15 Gyr against the observed LMC spectrum. Both spectra are normalized at $4810 \AA$ (arbitrary value). . . . . . . . . . . .

2.12 Contribution of the 3 phases of the 3 -phase scenario to the total spectrum. Top: Absolute contribution (i.e., summation of the 3 subpopulation spectra gives the total spectrum). Bottom: Subpopulation spectra and total spectrum normalized at $4810 \AA$ (arbitrary value). . . . . . . . . . . . . . . . . . 24

2.13 Variations of the 3-phase scenario (cf. Fig. 2.10): Scenarios with systematic variations of the SFH within phase $1(a, b)$, phase 2 $(c, d)$, and phase $3(e, f)$; note that the relative distribution of the total amount of SF between the 3 phases remains unchanged.

2.14 Variations of the 3-phase scenario (cf. Fig. 2.10): Scenarios with twice and half the SF, respectively, within phase $1(g, h)$, phase $2(i, j)$, and phase $3(k, l)$; the relative distribution of the total amount of SF between the 3 phases is changed. . . . . . . . .

2.15 Left: CMD and SFH as presented by Smecker-Hane et al. (2002). Right: Model CMD at a simulated galaxy age of 15 Gyr, using Smecker-Hane et al.'s SFH. Stellar populations originating from 4 different phases of star formation are coded in different colours (cf. the electronic version of this paper). . . . . . . . . . . . .

2.16 Confrontation of scenarios using Smecker-Hane at al.'s (2002) original SFH (left panels), a simplified Smecker-Hane SFH (central panels), and using a simple 3-phase SFH (right panels); see text.

Top: Model spectra of the scenarios after 15 Gyr (black) against observed spectrum (grey). Bottom: Photometric evolution of the scenarios in terms of $(\mathrm{B}-\mathrm{V})_{H S T},(\mathrm{~V}-\mathrm{R})_{H S T}$, and $(\mathrm{B}-\mathrm{R})_{H S T}$ with the corresponding SFHs; the observed colours (obtained from the observed spectrum) are marked with black dots at 15 Gyr. . . . .

2.17 Confrontation of scenarios using Smecker-Hane et al.'s (2002) original SFH (left panels), a simplified Smecker-Hane SFH (central panels), and using a simple 3-phase SFH (right panels); see text.

Top: Model CMDs at a simulated galaxy age of 15 Gyr. Bottom: Corresponding SFHs. . . . . . . . . . . . . . . . . . . 
3.1 Indices $\mathrm{H} \beta$ (left) and $\mathrm{Fe} 5335$ (right) versus metallicity for 5 different ages. Also shown are Galactic GC observations from various authors as indicated in the right-hand panel; GC metallicities are taken from Harris (1996, revision Feb. 2003). A typical measure-

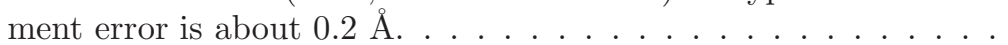

3.2 Indices $\mathrm{H} \beta$ (left) and $\mathrm{Fe} 5335$ (right) versus age for 6 different metallicities. Also shown are Galactic GC observations from various authors as indicated in the right-hand panel; GC age determinations are taken from Salaris \& Weiss (2002). . . . . . . . . .

3.3 Absolute differences of index strengths for old and young SSP models for changing metallicity $(t o p)$, and for metal-rich and metal-poor SSP models for changing age (bottom). The dotted lines are just for presentation. . . . . . . . . . . . . .

3.4 Galactic GC observations: Metallicities (left) and ages (right) determined using our Lick index analysis tool (x-axis, using all measured indices available) vs. metallicities and ages determined by CMD analyses (y-axis, taken from Salaris \& Weiss 2002). Note that only results with model uncertainties of $\sigma$ (age) $\leq 5$ Gyr are plotted. ....................

3.5 Same as Fig. 3.4, but using metallicity-sensitive indices $\mathrm{Mg}_{1}$, $\mathrm{NaD},[\mathrm{MgFe}]$, plus age-sensitive index $\mathrm{H} \beta$ as input only (left), and using age-sensitive indices $\mathrm{Ca} 4227, \mathrm{G} 4300, \mathrm{H} \beta$, and $\mathrm{TiO}_{1}$ as input only $($ right $) . \ldots \ldots \ldots \ldots$

3.6 Top: Lick index measurements of the Galactic GC M3 (NGC 5272 ) by Trager et al. (1998) with observational errors (open circles) and "best model" indices with the $\pm 1 \sigma$ confidence intervals (black dots). The best model has an age of $12.88\left(\begin{array}{c}-1.99 \\ +1.75\end{array}\right) \mathrm{Gyr}$ and $[\mathrm{Fe} / \mathrm{H}]=-1.7( \pm 0)$ dex; Salaris \& Weiss (2002) give age $=$ $12.1( \pm 0.7)$ Gyr and $[\mathrm{Fe} / \mathrm{H}]=-1.66$ dex.

Bottom: Lick index measurements of the Galactic GC M4 (NGC 6121) by Beasley et al. (2004) with observational errors (open circles), and "best model" indices with the $\pm 1 \sigma$ confidence intervals (black dots). The best model has an age of only $4.59\left(\begin{array}{c}-0.31 \\ +0.80\end{array}\right)$ Gyr and $[\mathrm{Fe} / \mathrm{H}]=-1.2\left(\begin{array}{c}-0.1 \\ +0.0\end{array}\right)$ dex; Salaris \& Weiss (2002) give age $=11.9( \pm 1.1)$ Gyr and $[\mathrm{Fe} / \mathrm{H}]=-1.27$ dex. Additionally, we plot model indices for the Salaris \& Weiss (2002) solution, i.e., an 11.9 Gyr / $[\mathrm{Fe} / \mathrm{H}]=-1.3$ dex SSP model (small crosses). . .

3.7 M31 GCs: Metallicities and ages for the Beasley et al. (2004) GC sample, determined using our Lick index analysis tool (x-axis, using all measured indices available) vs. metallicity determinations taken from Barmby et al. (2000) (top panel) and Puzia et al. (2005) (middle panel), and vs. age determinations taken from Puzia et al. (2005) (bottom panel). The classification as "young cluster" and "suspected dwarf galaxy" is taken from Beasley et 
3.8 M31 GCs: Absolute differences between parameters derived using our Lick index analysis tool and results from Puzia et al. (2005), against $[\alpha / \mathrm{Fe}]$ taken from Puzia et al. (2005). Left: $\left([\mathrm{Z} / \mathrm{H}]_{\text {Puzia }}-[\mathrm{Fe} / \mathrm{H}]_{\text {our models }}\right) ;$ Right: $\left(\right.$ age $_{\text {Puzia }}-$ age $\left._{\text {our models }}\right)$. The classification as "intermediate-age cluster" and "suspected dwarf galaxy" is taken from Beasley et al. (2004). . . . . . . . . .

4.1 Ages (left) and metallicities (right) for 11 LMC star clusters analysed using broad-band photometry UBVJHKs for AnalySED (top), the 5 Lick indices $\mathrm{H} \beta, \mathrm{Mg} b, \mathrm{Mg}_{2}, \mathrm{Fe} 5270$, Fe5335 for the Lick analysis (middle), and PRODUCT using both sets of data (bottom), plotted against ages and metallicities given in the literature (y-axes). Note that the age given in the literature for cluster NGC 1916 (blue circle) is very unsure (see text). . . . . . 63

4.2 Same as Fig. 4.1, but using UBV only for AnalySED. . . . . . . 64

4.3 Same as Fig. 4.1, but using Lick indices $\mathrm{H} \beta$ \& $[\mathrm{MgFe}]$ only for the Lick analysis. . . . . . . . . . . . . . . . . . . . . . 65

4.4 Same as Fig. 4.1, but using UBV only for AnalySED, and $\mathrm{H} \beta$ \& $[\mathrm{MgFe}]$ only for the Lick analysis. . . . . . . . . . . . . 66

4.5 Age-metallicity relation of our LMC cluster sample. Black dots give literature values, green crosses our best results obtained by analysing UBVJHK photometry together with the 5 Lick indices $\mathrm{H} \beta, \mathrm{Mg} b, \mathrm{Mg}_{2}, \mathrm{Fe} 5270$, and Fe5335 using PRODUCT. Cluster NGC 1916 is marked by a circle around the dot and the cross, respectively. . . . . . . . . . . . . . . .

4.6 Best models for NGC 5128 globular cluster pff_gc-006 using three different methods of analysis (data: Peng et al. 2004). The plots show the normalized probability space resulting from an analysis of the broad-band magnitudes UBVRI (a), the Lick indices $\mathrm{H} \beta$, $\mathrm{Mg} b, \mathrm{Mg}_{2}, \mathrm{Fe} 5270, \mathrm{Fe} 5335$ (b), and of a combination of both methods using PRODUCT (c). . . . . . . . . . . .

4.7 Left: Histogram of differences between age determinations using AnalySED and Lick Analysis, respectively, and using PRODUCT for 135 GCs in NGC 5128. Right: Same as left, but for metallicities. 71

4.8 Metallicities of star clusters in NGC 5128, determined by PRODUCT, vs U-V. Different symbols are used for two age bins. Also shown are GALEV model SSPs for 5 different ages and a colour metallicity relation taken from Barmby et al. (2000). . . . . . . .

5.1 Positions of all clusters for which UBVRI photometry is available, overplotted on a DSS image of NGC 5128 (left panel; taken from Peng et al. 2004). The right panel reproduces this figure, but also show the position of the subsample of clusters for which spectral indides are available as well. . . . . . . . . . . . . . . .

5.2 Histograms of dereddened colours U-V (left panel), B-V (middle panel), and V-I (right panel) for the complete sample of clusters (black histogram), and for the subsample of clusters with spectral observations (green shaded area). . . . . . . . . . . . . .

5.3 Metallicity (left) and age (right) distribution of the NGC 5128 cluster sample. . . . . . . . . . . . . . . 
5.4 Age vs metallicity for the NGC 5128 cluster sample. Three different subpopulations are emphasized by boxes. . . . . . . . . 85

5.5 Positions of all cluster for the three populations defined in the text (black triangles): Population 1 (left panel, population 2 (middle panel), and population 3 (right panel). To be compared with Fig. 5.1 , the complete sample of clusters analysed is plotted as well (green stars). . . . . . . . . . . . . . 86 


\section{List of Tables}

2.1 Characteristics of the SF scenarios to be compared; the enhancement of SF always refers to a "basic rate" of $1 \mathrm{M}_{\odot} /$ yr. Scnrs. 0-6: ongoing SF after 6.4 Gyr $\left(1 \mathrm{M}_{\odot} / \mathrm{yr}\right)$; scnrs. 0b-6b: SF truncated after 6.4 Gyr. . . . . . . . . . . . . . . . .

2.2 Integrated colours in $\mathrm{U}-\mathrm{V}, \mathrm{V}-\mathrm{I}, \mathrm{V}-\mathrm{K}$ for SSP models with different age metallicity combinations, arranged in three pairs with equal or very similar colour in $\mathrm{V}-\mathrm{I}$. . . . . . . . . . . . . . . . . . 20

2.3 Colours derived from the integrated-light spectrum of the LMC bar field, obtained by folding the spectrum with the respective filter functions. . . . . . . . . . . . . . . . . .

3.1 Metallicity sensitivity parameters for different zero points. Low numbers indicate high age sensitivity. Values given in brackets are not reliable (see text). . . . . . . . . . . . .

3.2 "Neighbouring models" in terms of metallicity for $\mathrm{Z}=0.02$ and $\mathrm{Z}=0.0004($ top $)$ and in terms of age for the zero points $12 \mathrm{Gyr}$ and 4 Gyr (bottom) used to compute the metallicity sensitivities given in Table 3.1. Brackets give the corresponding $\Delta \mathrm{Z}$ and $\Delta$ age. 45

3.3 Observations by Burstein et al. (1984, B84), Covinio et al. (1995, C95), Trager et al. (1998, T98; $\mathrm{H} \delta, \mathrm{H} \gamma$ are taken from Kuntschner et al. 2002, see text), Beasley et al. (2004, B04) used to perform the tests of Sect. 3.4.2. '*': index observed, 'o': only a subsample of clusters is observed in this index. . . . . . . . . . . . . . . . 48

3.4 Mean ages and standard deviations of cluster ages determined using the Lick index analysis tool and CMD analysis (Salaris \& Weiss 2002), respectively, as shown in Figs. 3.4 and 3.5. Note that the values are computed without cluster NGC 6121. . . . . 51

4.1 Ages and metallicities for 11 LMC star clusters analysed using broad-band photometry UBVJHKs for AnalySED, the 5 Lick indices $\mathrm{H} \beta, \mathrm{Mg} b, \mathrm{Mg}_{2}$, Fe5270, Fe5335 for the Lick analysis, and PRODUCT using both sets of data. . . . . . . . . . . . 77

4.2 Same as Fig. 4.1, but using UBV only for AnalySED. . . . . . . 78

4.3 Same as Fig. 4.1, but using Lick indices $\mathrm{H} \beta$ \& $[\mathrm{MgFe}]$ only for the Lick analysis. . . . . . . . . . . . . . . . . . . . . . . . 79

4.4 Same as Fig. 4.1, but using UBV only for AnalySED, and $\mathrm{H} \beta$ \& $[\mathrm{MgFe}]$ only for the Lick analysis. . . . . . . . . . . . . 80 



\section{Chapter 1}

\section{Introduction \& Overview}

Galaxies are fascinating objects for astronomical studies. One of the most struggling questions in this field is the question for the formation processes and evolutionary histories of galaxies. Especially for elliptical galaxies, various scenarious have been developed which all are under discussion today.

In order to decide between different scenarious it is necessary to get reliable information about both the star formation history ( $\mathrm{SFH}$ ) and the chemical enrichment history of a galaxy. A variety of very different methods using very different kinds of data are available for doing this.

The method generally regarded as most reliable is the analysis of colour magnitude diagrams (CMDs), which can give relatively precise results for the SFH of galaxies. However, details of star formation (SF) recovered get worse for longer lookback times; moreover, the chemical enrichment history can only poorly be recovered. The most severe problem, however, is that CMD studies are restricted to local group galaxies, and, therefore, to a very limited sample of galaxy types. Moreover, CMDs can only be obtained when crowding is not severe; therefore, in the majority of cases only for the outskirts of galaxies can CMDs be obtained.

For galaxies far away the only information available is the integrated light emitted by the composite stellar population of a galaxy, in terms of photometry or spectra. In this thesis I show, however, that both photometric spectral energy distributions (SEDs) and spectra obtained for the integrated light of a galaxy can give only very vague hints about the distribution of SF over the lifetime of a galaxy. E.g., any details of SF during early stages of galactic evolution are completely smoothed over by a recent burst. Only for a very short timescale of 1 to 4 Gyrs some information can be recovered.

However, since star cluster formation is an important mode of SF, in particular during violent SF episodes, the age and metallicity distributions of globular cluster systems (GCSs) hold unique clues about the formation histories of their parent galaxies over cosmological timescales. Thus, GCs can be regarded as "frozen witnesses" of galactic evolution; a careful analysis can give informations about both the unique SFH and chemical enrichment history of a galaxy.

Within the framework of this thesis, I have developed new reliable tools for the analysis of GCSs which make use of all information available for a given set of star cluster observations: 
For the analysis of broadband SEDs, an analysis tool for the determination of ages and metallicities of star clusters is at hand (AnalySED).

However, since changes in the SEDs of star clusters older than about $3 \mathrm{Gyr}$ get slower and more and more subtle, age-dating and disentangling ages and metallicities gets more and more difficult if only photometric SEDs are analysed.

Therefore, I have incorporated spectral Lick/IDS indices into the evolutionary synthesis code GALEV (which is used for calculating all models presented in this thesis), and developed an analysis tool (Lick Analysis Tool) for the independent determination of age and metallicity of individual clusters, including mathematically reasonable and reliable $1 \sigma$ confidence intervals, using the complete set of indices available for analysis all at once.

However, even when using spectral information, results still suffer from agemetallicity degeneracy: Since metallicities proved to be very reliable using Lick index analysis, $1 \sigma$ uncertainties in age can still be very high (up to more than 10 Gyrs in worst cases). In order to further reduce the degeneracies inherent in each kind of dataset, I therefore developed a completely new method to combine the analysis of broadband colours (AnalySED) and Lick indices (Lick-Analysis) and, hence, to utilize all the information available in a mathematically reasonable way (PRODUCT).

The thesis is organized as follows:

In Chapt. 2, I explore the fundamental question, to what precision SFHs of galaxies can in principle be determined by analysing integrated colours and spectra, in comparision to CMDs, and present a methodological application to a star field in the bar of the LMC. This chapter has been submitted for publication in A\&A.

In the following two chapters I introduce new models as well as new advanced methods for the analysis of GCSs:

In Chapt. 3, I present new models for Lick indices which I have incorporated into the GALEV evolutionary synthesis code, and I present a mathematically advanced and reliable tool for the independent determination of age and metallicity of individual GCs, supplemented by extensive tests using Lick index data for both Milky Way and M31 GCs. I also adress the important issue of Lick index sensitivities. This chapter has already been published as Lilly and Fritze-v. Alvensleben, 2006, A\&A 457, 467.

In Chapt. 4, I present a method for the combined analysis of broad-band SEDs and spectral indices, PRODUCT, which allows to constrain ages and metallicities of individual GCs even in cases when poor datasets are available only. This chapter will be submitted shortly for publication in A\&A.

In Chapt. 5, I present applications of the models and analysis tools developed in the framework of this thesis to the GCS of the large elliptical galaxy NGC 5128 , using a dataset consisting of both broad-band photometry and Lick indices for an unprecedentedly large sample of GCs. An extended version of this chapter is in preparation for submission to $\mathrm{A} \& \mathrm{~A}$. 


\title{
Chapter 2
}

\section{Tracing back the star formation histories of galaxies:}

\section{A methodological study comparing integrated light and CMD studies, and applications to a field in the $\mathrm{LMC}^{1}$}

\begin{abstract}
Context.Integrated light data like colours or absorption line indices are widely used to get information about the evolutionary history of a galaxy's stellar content, e.g. about the strength of its latest burst, a galaxy's "mean age", or even about the overall distribution of star formation (SF) during its lifetime.

Aims. Our study aims at understanding to what precision star formation histories (SFHs) can in principle be determined for distant galaxies observable in integrated light only.

Methods. Using our evolutionary synthesis code, we have performed a set of simulations of galaxies with a wide range of different SFHs, but constant metallicity. By analysing the resulting colours and spectral indices, we investigate to which extent different $S F$ scenarios can be discriminated on the basis of their photometric and spectral properties, respectively. As a test object, we analyse integrated-light colours of a field in the LMC bar, for which highly resolved HST
\end{abstract}

${ }^{1}$ This chapter has been submitted for publication in A\&A as Lilly and Fritze-v. Alvensleben, 2007 
images are available as well. To be compared with the SFH derived from the colour magnitude diagram (CMD) of this field (Smecker-Hane et al. 2002), again we performe a set of simulations of galaxies with systematically varying SFHs and determine in how far the detailed SFH obtained by the CMD approach can be reproduced by results based upon integrated properties.

Results. We find the robust result that no later than 4 Gyrs after the latest episode of enhanced star formation all scenarios exhibit very similar colours and indices; in practice, it is not possible to distinguish different scenarios of star formation which have evolved for more than 1, at the utmost 3-4 Gyrs since the last star forming event, even when using spectral indices. For how long different SF scenarios can be disentangled highly depends on the range of colours available and absorption lines considered, as well as on the details of the SFHs to be compared. We show that the integrated colours obtained for the LMC field, which only cover a very small wavelength basis, can be reproduced by a very simple "toy model" consisting of three different phases of constant SF only.

\section{$2.1 \quad$ Introduction}

"It is startling to realize that after only $\sim 10^{9}$ yr galaxies like these will resemble ordinary ellipticals in both morphology and colors!"

Dealing with galaxies "with colors suggesting that a large burst of star formation occured a few times $10^{8}$ yr ago", the above statement was given almost 30 years ago by Beatrice Tinsley (1978), the pioneer of evolutionary synthesis modelling. She continued that this result "is due to the insensitivity of (...) colors to parameters other than the total age and the star formation rate in the last $10^{9}$ yr."

Today, 30 years later and after impressive advancements in both observational and modelling techniques which allow to observe as well as to simulate integrated colours and spectra of galaxies to a degree of precision much higher than this was possible at Tinsley's time, the question arises: Is the situation as "startling" as thirty years ago? Can we, using the tools available today, recover details of past star formation (SF) in a galaxy with better accuracy and for lookback times much longer than the $10^{9}$ yr mentioned by Tinsley?

Still, methods aiming at the reconstruction of the star formation history (SFH) of a galaxy are important tools for understanding the evolution of these objects. The available methods can be devided into two groups: Methods using colour magnitude diagrams (CMDs) are widely regarded as the most reliable ones. However, they require that the stellar population can be resolved into individual stars; therefore, methods of this kind are limited to nearby galaxies, and there only to star fields without crowding. The second group of methods makes use of integrated-light properties of unresolved galaxies like colours and spectra, which are far easier to get for a much wider range of galaxies, even for those at high redshift. Integrated colours and spectra can be analysed and interpreted by means of either population synthesis codes or evolutionary synthesis codes like the GALEV code used in this paper.

Much work has been done in testing these codes with respect to internal errors, to the influence of the input physics used, and to the degree of agreement/disagreement among each other (see, e.g.: Charlot et al. 1996; Cerviño 
et al. 2000 et seqq.; Bruzual 2001; Yi 2002). In addition, differences between observed and model-predicted properties of galaxies and star clusters have been investigated in detail (see, e.g.: Vazdekis et al. 2001; Schulz et al. 2002).

In this work, we deal with a quite different but related question: While ignoring the already well explored model-dependent uncertainties mentioned above, we want to answer the fundamental question, to what precision SFHs of galaxies can in principle be determined by using integrated light only, that is, independent of the model but dependent on the physical properties of a galaxy's stellar population. Beyond the performance of other evolutionary synthesis codes, our GALEV code in addition offers the possibility to study the evolution of any of the stellar population models (star clusters, galaxies with arbitrary $\mathrm{SFH}$ ) in terms of CMDS. In fact, the spectral evolution of the integrated light is calculated on the basis of the evolution of the stellar populations across the $\mathrm{HR}$ diagram, and the photometric evolution is calculated on the basis of the spectral evolution by folding responding functions for any desired filter system and detector with the model spectra. Hence, GALEV also offers the possibility to compare various SFHs in terms of CMDs and integrated light.

Using our evolutionary synthesis code GALEV, we have therefore performed a set of simulations of galaxies with a wide range of different SFHs but well defined and uniform input physics. That way, we restrict our investigation to influences of the variation of the SFH on the spectro-photometric properties of a galaxy; other parameters like the initial mass function or the metallicity are kept constant in this study for clarity.

By confrontation of the evolution of the colours and spectra resulting from the various simulations we then investigate, to which extent different SF scenarios can be discriminated at all and as a function of lookback time, and how this depends on the details of the assumed SFHs. For clarity we restrict our study to simplified SFHs with constant star formation rates (SFRs) over various periods of time, from long intervals of low SFR to starburst periods of high SFR. Because the models are all synthesised using one genuine code and one genuine set of input physics, we can perform this comparative study in a self-consistent way.

In a second step, we present our work within a larger collaborative project with the aim to confront different methods to derive SFHs from integrated light against each other and against the CMD approach. The motivation for this collaborative study comes from the fact that while only for a few very nearby systems SFHs can be derived from CMD analyses, a method well established and carefully tested on Galactic star clusters, all that is accessible for distant galaxies is information that can be derived from integrated light - spectra or multi-band photometry.

Test object for this ongoing project is one specific field in the bar of the Large Magellanic Cloud (LMC), for which both an integrated-light spectrum (obtained with the ESO 3.6m telescope, LaSilla) and data on its resolved stellar population (obtained with the Hubble Space Telescope, HST) are available. That way, the results of the different groups analysing the spectrum can not only be compared with each other but can also be compared with the SFH obtained by an analysis of the CMD of the same field. A short description of the project can be found in Alloin et al. (2002); an analysis of the CMD for this field is presented by Smecker-Hane et al. (2002). 
Here, we present parts of our analysis of the integrated-light spectrum of the LMC bar field. Applying the results of our preparatory work presented in the first part of this paper, we again performed a set of simulations of galaxies with systematically varying SFHs, starting with a toy model having only three different phases of constant SFR. The colours and spectra resulting from the various simulations are then confronted against the observations as well as against each other to investigate in how far the detailed SFH obtained by the CMD approach can be retrieved from integrated properties. Like before, we keep other parameters, like the initial mass function or the metallicity, constant.

It should be kept in mind that, due to the character of the study, it is by no means to be regarded as an attempt to recover the real physical SFH of the LMC, nor as a practical instruction of how to recover the SFH of a given galaxy, although, as we hope, much can be learned about how to do this most efficiently, e.g. by adressing the question what filters or spectral lines should be analysed, and in how far the results can be reliably interpreted.

\subsection{Models and input physics}

Our evolutionary synthesis models GALEV describe the spectrophotometric evolution of the integrated light of large stellar populations like galaxies or star clusters. They are 1-zone models, i.e. the spatial resolution and the dynamical properties remain unconsidered. As for all evolutionary synthesis models, the (historical) basis of the code are the equations given by Tinsley (e.g., 1980), which describe the global balance of stars and gas in a galaxy, and kind of a book-keeping algorithm that keeps track of all stars in a model galaxy at various timesteps and the evolution of their distribution over the HR diagram.

Input physics for the code include the theoretical spectral library from Lejeune et al. $(1997,1998)$ as well as theoretical isochrones from the Padova group like the ones described by Bertelli et al. (1994) for 5 different metallicities $Z=0.0004,0.004,0.008,0.02$ and 0.05 , but in the version from November 1999 that includes the TP-AGB phase of stellar evolution (as described in Schulz et al. 2002). We assume a standard Salpeter (1955) initial mass function (IMF) from 0.15 to about $70 \mathrm{M}_{\odot}$; the lowest mass stars $\left(\mathrm{M}_{\odot}<0.6\right)$ are taken from Chabrier \& Baraffe (1997) (cf. Schulz et al. 2002 for details). Since the resolution of Lejeune et al's spectral library does not allow to measure Lick indices on the spectra, we use the polynomial fitting functions of Worthey et al. (1994) and Worthey \& Ottaviani (1997), which give Lick index strenghts of individual stars as a function of their effective temperature $T_{\text {eff }}$, surface gravity $g$, and metallicity $[\mathrm{Fe} / \mathrm{H}]$ as the basis for our models for Lick indices. Worthey et al. have calibrated their fitting functions empirically using Milky Way stars.

Once an IMF is assumed, the basic free parameters of our models are the star formation rate (SFR) and the metallicity of the stellar population; for this specific study we restrict ourselves to isochrones for half-solar metallicity $Z=$ 0.008 and, for simplicity, ignore any successive enrichment and the presence of several metallicity subpopulations in galaxies, i.e. we do not make use of the chemically consistent GALEV models presented by Bicker et al. (2004). The code then produces the time evolution $(4 \mathrm{Myr} \ldots 16 \mathrm{Gyr})$ of spectra $(90 \AA \ldots$ $160 \mu \mathrm{m}$ ), colours in many filter systems (from UV to NIR), and 25 Lick/IDS 
spectral indices.

For an exhaustive description of GALEV and its input physics see Schulz et al. (2002), Bicker et al. (2004), Anders \& Fritze-v. Alvensleben (2003), and Lilly \& Fritze-v. Alvensleben (2006).

The GALEV code is unique among evolutionary synthesis codes in that it also calculates the time evolution of its stellar populations in terms of CMDs of arbitrary filter combinations. CMDs are produced in a very direct way (cf. Lilly 2003): The number of stars at each point on the isochrones is determined by the IMF and the relative weight of the isochrone computed by GALEV (which is, in fact, the basis of our description of the integrated light and its evolution). The stars are spread around their theoretical position on their isochrone, following a Gaussian distribution, by applying typical observational errors in colour and magnitude, respectively, small for bright stars and larger for fainter stars. Throughout this paper, we assume the typical observational errors obtained by HST observations of a star field in the bar of the LMC (cf. Sect. 2.4.3), as given in Smecker-Hane at al. (2002).

For any given SFH (and chemical enrichment history) our code is able to calculate the time evolution of the distribution of stars in the HR diagram and any desired CMD. However, we do not interpolate between isochrones; therefore, we had to increase the assumed observational errors in magnitude in order to reduce the "gaps" between isochrones on the CMD (cp. observed with model errors as shown in Fig. 2.15).

Hence, our model CMDs are not intended to be directly compared with observations but, so far, for principle investigations only (model-model comparisons).

\subsection{Scenarios in integrated light}

We want to explore in how far different SF scenarios can in retrospect be discriminated against each other. Therefore, we have performed a set of simulations of galaxies with a range of different SFHs chosen to be instructive rather than necessarily realistic. Other parameters are kept constant for clarity; for all scenarios, we assume a Salpeter (1955) IMF and a fixed metallicity of $Z=0.008$. The initial amount of gas is chosen large enough in all cases to ensure that there is always enough gas available for SF, so that the galaxy cannot 'burnout'.

For simplicity and lack of better knowledge about realistic burst shapes we assume rectangular burst shapes on top of constant SF (we do not expect this simplification to substantially affect on results). Unless stated otherwise, we assume that any two SF scenarios we compare have produced the same amount of stars in total. Therefore, the absolute numerical value of the SFR does not matter for the colours or spectral indices of the integrated light. It is the relative distribution of the SFR over the evolutionary time of the galaxy that is significant.

The characteristics of all SF scenarios compared in this section can be found in Table 2.1. For each scenario, there are two different versions: In Scnrs. 0-6, SF goes on after 6.4 Gyrs, in scnrs. 0b-6b (the "b-scenarios") the SF is truncated after 6.4 Gyr. Note that, throughout this paper, age follows galactic evolution 
Table 2.1: Characteristics of the SF scenarios to be compared; the enhancement of SF always refers to a "basic rate" of $1 \mathrm{M}_{\odot} / \mathrm{yr}$. Scnrs. 0-6: ongoing SF after 6.4 Gyr $\left(1 \mathrm{M}_{\odot} / \mathrm{yr}\right)$; scnrs. 0b-6b: SF truncated after 6.4 Gyr.

\begin{tabular}{|c|c|}
\hline scenario & description \\
\hline $0 / 0 b$ & constant SF rate (factor 3.4) from $t=0$ Gyr through $t=6.4$ Gyr \\
\hline $1 / 1 \mathrm{~b}$ & $\begin{array}{l}2 \text { bursts (SF enhanced by a factor of } 20 \text { ) of duration } 0.4 \text { Gyr at } t=4 \\
\text { Gyr and } t=6 \mathrm{Gyr}\end{array}$ \\
\hline $2 / 2 b$ & enhanced SF (factor 7 ) from $t=4$ Gyr through $t=6.4 \mathrm{Gyr}$ \\
\hline $3 / 3 b$ & $\begin{array}{l}2 \text { bursts (SF enhanced by a factor of } 20 \text { ) of duration } 0.4 \text { Gyr at } t=0 \\
\text { Gyr and } t=6 \text { Gyr }\end{array}$ \\
\hline $4 / 4 b$ & $\begin{array}{l}1 \text { bursts (SF enhanced by a factor of } 20 \text { ) of duration } 0.8 \text { Gyr at } t=5.6 \\
\text { Gyr }\end{array}$ \\
\hline $6 / 6 \mathrm{~b}$ & $\begin{array}{l}1 \text { strong burst (SF enhanced by a factor of } 40 \text { ) of duration } 0.4 \text { Gyr at } \\
t=6 \mathrm{Gyr}\end{array}$ \\
\hline
\end{tabular}

(not lookback time); thus, a galaxy age of 6.4 Gyr refers to a galaxy which has evolved for 6.4 Gyrs, starting its evolution at 0 Gyr.

\subsubsection{Colours}

First, we confront the photometric evolution of four scenarios, each of them featuring one single burst of SF, ranging from a strong burst at 6 Gyr galaxy age (scenario 6) through bursts with weaker strengths but longer durations (scenarios 4 and 2), to a case of an extended phase of only slightly enhanced SF (scenario 0). In each case, the bursts are put on top of a very low but constant "basic rate" of SF of $1 \mathrm{M}_{\odot} / \mathrm{yr}$, and all "bursts" finish at 6.4 Gyr galaxy age. In all these scenarios, the "basic rate" of SF goes on after 6.4 Gyrs for the remaining life of the galaxy.

We assume a typical photometric accuracy of about $0.1 \mathrm{mag}$, at best 0.05 mag. This means, if differences in model-predicted colours between different scenarios of SF do not exceed $0.1 \mathrm{mag}$, they will be considered as observationally indistinguishable.

Fig. 2.1 (left panels) shows the photometric evolution of our scenarios. To guide the eye, we plot a thick line at $6.4 \mathrm{Gyr}$. The bottom plots show the respective SFHs to be compared. The plots show impressively that already 1 Gyr after the latest enhanced SF period all scenarios show nearly identical colours. Even though scenario 0 differs in $\mathrm{U}-\mathrm{V}$ from the other scenarios for at least 4 Gyrs due to the larger amount of still existing red giant stars originating from the phases of enhanced or 'bursty' SF in the latter, this feature can hardly be used for the reconstruction of SFHs because the maximum difference of 0.1 mag declines rapidly to practically indistinguishable values of about 0.05 mag.

Hence, the "lookback time", during which bursts of different strengths can be discriminated in integrated light is only 1 , at the utmost 4 Gyr; after that time, it is not even possible to discriminate between a strong burst scenario of galaxy evolution and a very "quiet" evolution like that of scenario 0 , if no other clues about a possibly violent history of the galaxy are available but only global 

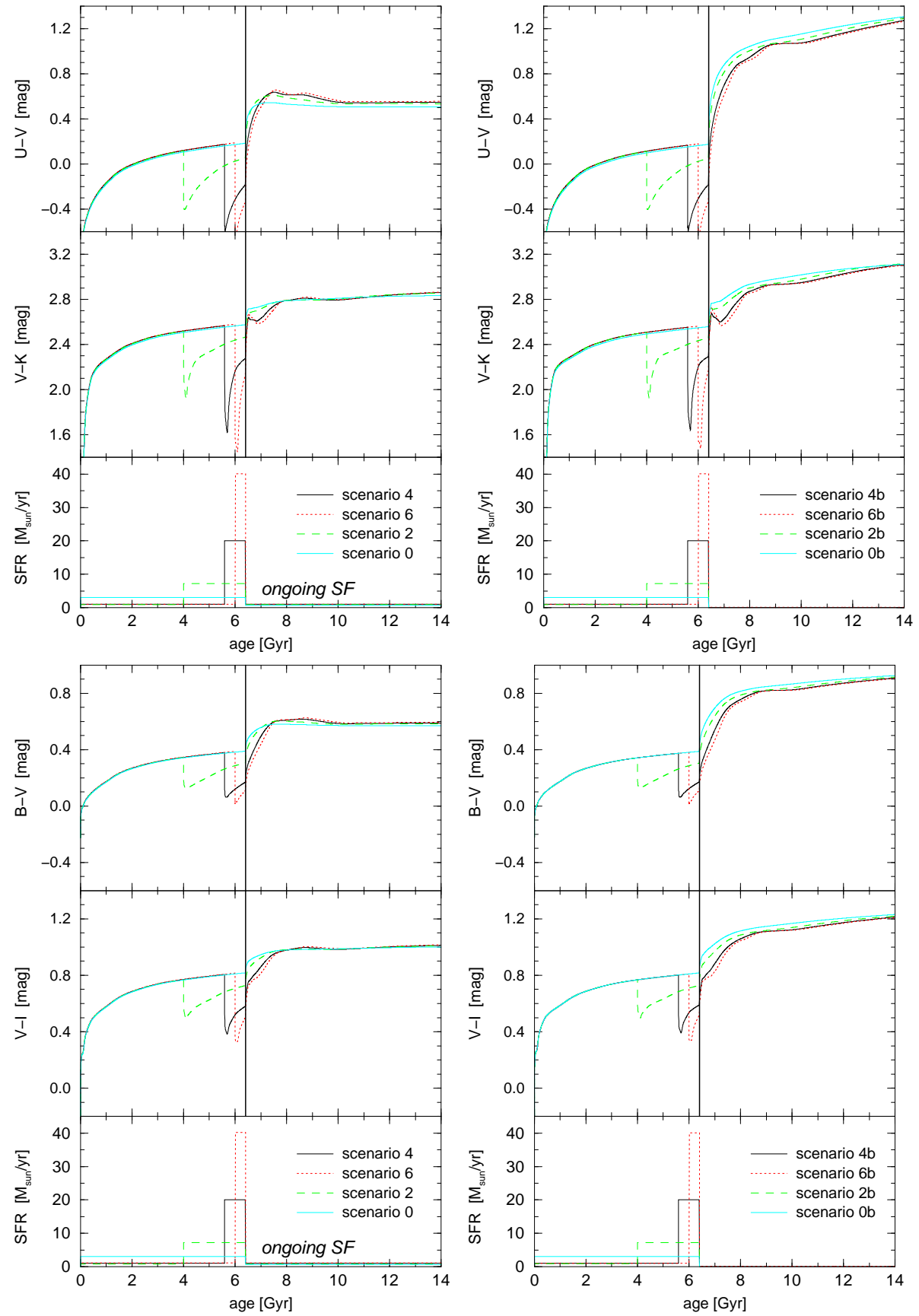

Fig. 2.1: Confrontation of the photometric evolution of scenarios $0,2,4,6$ (left) and $0 \mathrm{~b}, 2 \mathrm{~b}, 4 \mathrm{~b}, 6 \mathrm{~b}$ (right), respectively, in terms of the colours $\mathrm{U}-\mathrm{V}, \mathrm{V}-\mathrm{K}$ (top), and B-V, V-I (bottom), respectively. At 6.4 Gyr, when the latest phase of enhanced SF ends, a thick line is plotted. The bottom plots show the respective SFHs to be compared. 

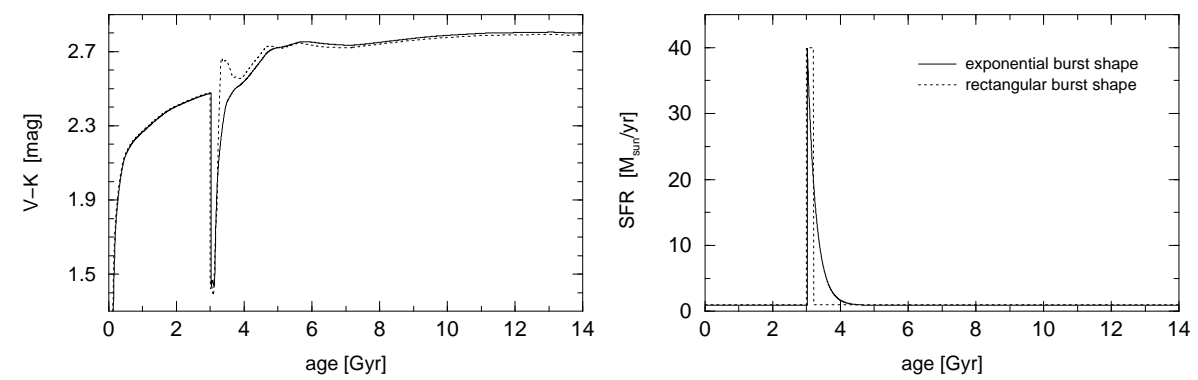

Fig. 2.2: (Left:) Photometric evolution in $\mathrm{V}-\mathrm{K}$ of a 'realistic' starburst (solid line) as compared to a rectangular one (dotted line). (Right:) Respective SFHs. See text for details.

broad-band colours.

Since any two SF scenarios we compare have produced the same amount of stars in total, the amount of long-living low-mass stars is roughly the same in all scenarios after the end of the most recent burst; therefore, the photometric distinction between different SF scenarios is mainly determined by luminous high-mass stars and their evolution. During a burst, for example, according to the IMF many more cool and red low-mass stars are formed than blue highmass stars. However, due to their extremely high luminosities, and despite their considerably smaller number, the massive stars dominate the integrated light, resulting in an abrupt change of colour in the model galaxy as soon as the burst starts.

As the bluest massive stars die out after the end of the burst, colours get redder very fast. This "reddening" can already be observed during bursts: Whereas the number of very blue and very massive stars reaches some equilibrium early in the burst, the redder low-mass stars accumulate during the burst (as well as during the whole lifetime of the galaxy) due to their considerably longer lifetimes, and therefore begin to overbalance the bluest high-mass stars.

These effects can be observed in all colours; they are stronger in colours covering a large spectral range like $\mathrm{U}-\mathrm{V}$ or $\mathrm{V}-\mathrm{K}$ than in colours from two close passbands like $\mathrm{B}-\mathrm{V}$ and $\mathrm{V}-\mathrm{I}$.

However, the difference between the scenarios is not larger in $\mathrm{V}-\mathrm{K}$ than in $\mathrm{V}-\mathrm{I}$; this is explained by intermediate-mass stars (stars with initial masses of $2 M_{\odot} \leq m \leq 7 M_{\odot}$ ) passing through the TP-AGB (thermally pulsing asymthotic giant branch) phase from an age of $10^{8} \mathrm{yr}$ up to an age of $10^{9} \mathrm{yr}$. During this phase, they are located in the upper right of the HR diagram and account for approx. 40-60\% of the K-band light (Lançon 1999). After each burst a typical bump in $\mathrm{V}-\mathrm{K}$ caused by TP-AGB stars can easily be identified; a comparison with a model that does not include the TP-AGB phase can be found in Schulz et al. (2002, Fig. 1).

Whether this bump occurs in $\mathrm{V}-\mathrm{K}$ also depends on the shape of the declining phase of the burst. In Fig. 2.2, we compare an exponentially declining burst (decay time $\tau=2.5 \cdot 10^{8} \mathrm{yr}$; for the burst model implemented here cf. Bicker et al. 2002) with a rectangular shaped one of equal strength (lasting $\sim 210$ Myrs, 
so that the same number of stars is formed in both cases); both bursts start at a galaxy age of 3 Gyr. Fig. 2.2 shows that in the case of an exponentially declining burst, the influence of the TP-AGB phase is much weaker than in the 'abruptly declining' burst model used in the simulations for this paper, resulting in a much better discernability between burst and non-burst scenarios during the declining phase. This can be explained by considering that in the exponentially declining burst phase the photometric influence of the TP-AGB stars is diluted by red supergiants still forming during the same period. Due to the extremely short lifetimes of these very massive red supergiants, they are less important shortly after a rectangular burst.

Hence, in "real" galaxy evolution scenarios we expect V-K to be a better indicator of former SF as V-I. The lookback time, however, over which any two SFHs can be discriminated from each other, is not longer in $\mathrm{V}-\mathrm{K}$ than in $\mathrm{V}-\mathrm{I}$.

Fig. 2.1 (right panels) shows the same scenarios in their "B-Versions": Here, the SF is truncated after the latest phase of enhanced SF, i.e. after $6.4 \mathrm{Gyr}$ galaxy age. In this case, the differences between the scenarios are slightly larger and can be noticed for a much longer time (about 7 Gyrs); however, the colourdifference even between non-burst scenario $0 \mathrm{~b}$ and scenario $6 \mathrm{~b}$ (which exhibits the strongest burst) is not larger than $0.1 \mathrm{mag}$, and after 7 Gyrs all colours are almost indentical $(\Delta(\mathrm{U}-\mathrm{V})<0.05 \mathrm{mag})$.

In Fig. 2.3 we confront the photometric evolution of three scenarios, each of them featuring a burst going on between 6.0 and 6.4 Gyr with a SFR of 20 $\mathrm{M}_{\odot} / \mathrm{yr}$ on top of galaxy models with different former SFHs: Scenarios 1 and 3 feature a previous burst with similar characteristics at a galaxy age of 4 Gyr, and at the beginning of galactic evolution (0 Gyr galaxy age), respectively. In scenario 4 , the two bursts are replaced by one single burst lasting twice as long (i.e., for 800 Myrs), starting at 5.6 Gyr galaxy age. Again, all scenarios form the same amount of stars in total.

The colour evolution plots in Fig. 2.3 show that on the basis of broad-band colours alone, all these scenarios are practically indistinguishable directly after the end of the most recent burst, even in the "b-scenarios" where the SF is truncated after 6.4 Gyr.

We conclude that after a burst, we are no longer able to detect any details in the SFH prior to this event on the basis of broad-band colours; all these details are completely smoothed over by the most recent burst. We cannot even discriminate a constant SFR from another burst or a series of previous ones with reasonable accuracy for a lookback time larger than about 1 Gyr. 

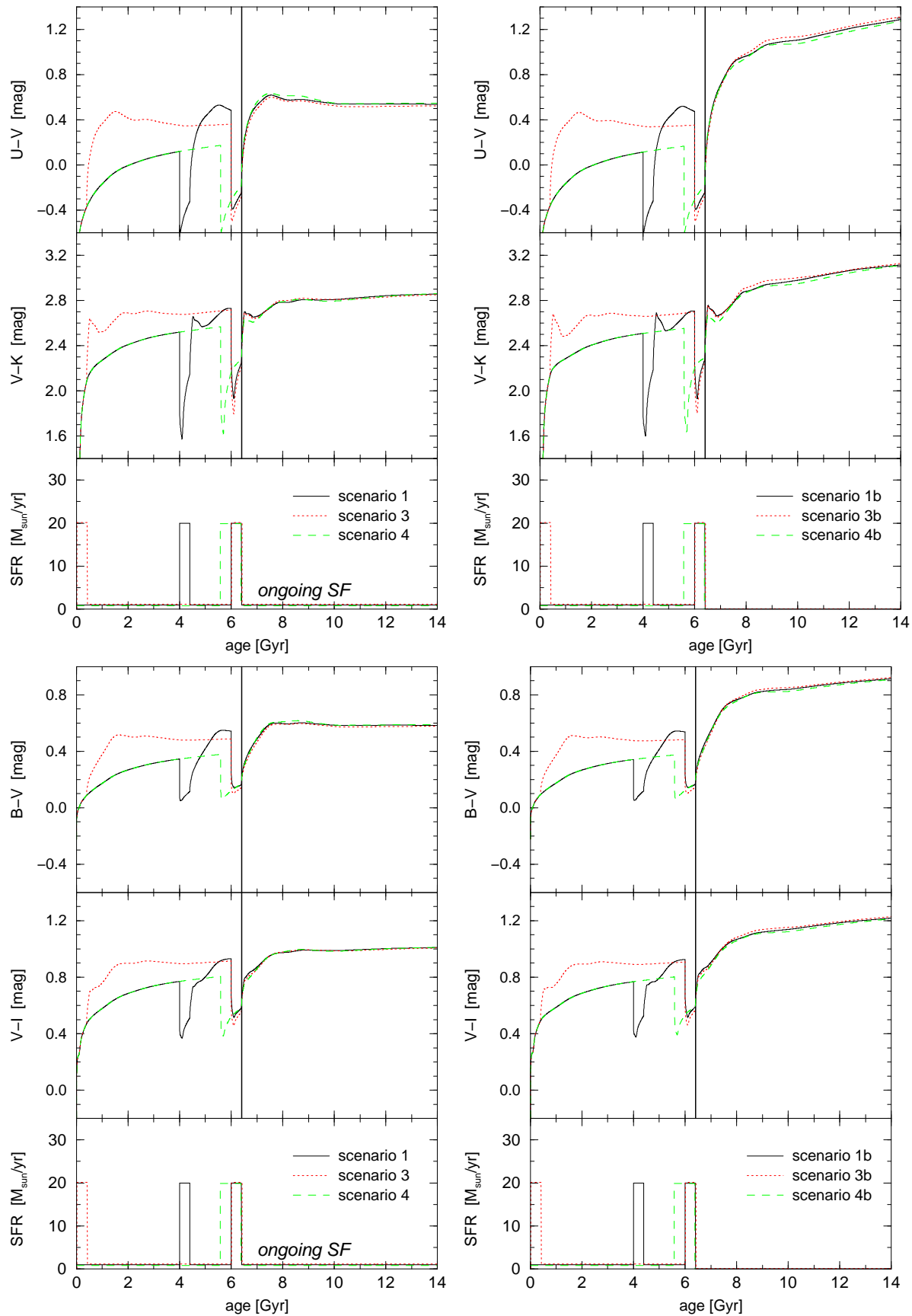

Fig. 2.3: Confrontation of the photometric evolution of scenarios 1, 3, 4 (left) and $1 \mathrm{~b}, 3 \mathrm{~b}, 4 \mathrm{~b}$ (right), respectively, in terms of the colours $\mathrm{U}-\mathrm{V}, \mathrm{V}-\mathrm{K}($ top $)$, and B-V, V-I (bottom), respectively. At $6.4 \mathrm{Gyr}$, when the latest phase of enhanced $\mathrm{SF}$ ends, a thick line is plotted. The bottom plots show the respective SFHs to be compared. 

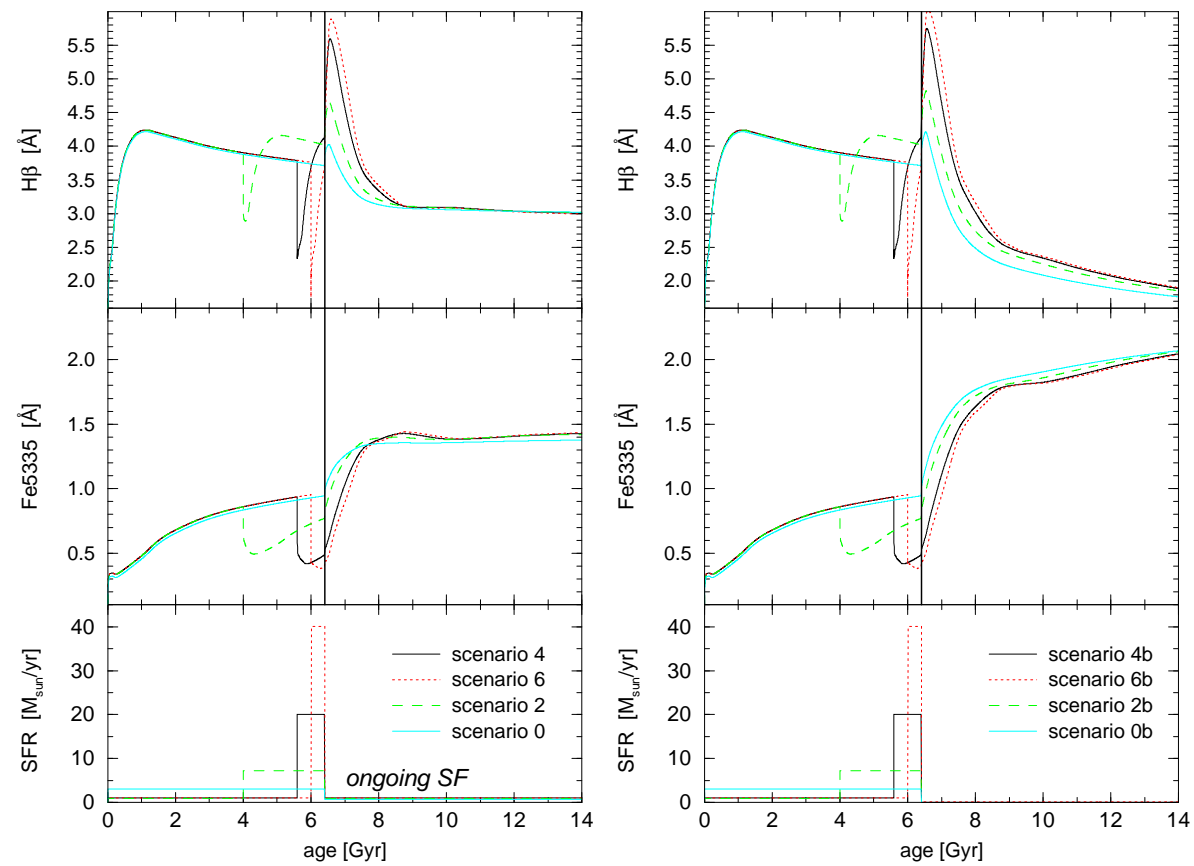

Fig. 2.4: Confrontation of the evolution of scenarios $0,2,4,6$ (left) and 0b, 2b, 4b, 6b (right), respectively, in terms of the Lick indices $\mathrm{H} \beta$ and Fe5335. At 6.4 Gyr, when the latest phase of enhanced SF ends, a thick line is plotted. The bottom plots show the respective SFHs to be compared.

\subsubsection{Lick indices}

In the last Section, we have seen that not even bursts with very different SFRs can be discriminated very well for more than 1, at the utmost 4, Gyr of lookback time in terms of colours. Now we want to explore whether the situation improves if Lick spectral indices are considered instead of broad-band colours. In this case, the typical accuracy of Lick index measurements is assumed to be about $0.2 \AA$, at best $0.1 \AA$, i.e. we consider two SF scenarios distinguishable from each other in terms of Lick indices as long as they differ by $\geq 0.2 \AA$, at least $\geq 0.1 \AA$.

Here, we again confront the evolution of scenarios featuring bursts of different strength and duration (Fig. 2.4; cf. last section, Fig. 2.1), and of scenarios featuring bursts going on between 6.0 and 6.4 Gyr with equal strength each, but different former SFHs (Fig. 2.5; cf. last section, Fig. 2.3), but this time in terms of the Lick index $\mathrm{H} \beta$, which is known to be particularly sensitive to age, and of the Lick index Fe5335, which is known to be more sensitive to metallicity than to age (cf. Worthey 1994; Lilly \& Fritze-v. Alvensleben 2006). Since the scenarios vary only in SFH, but have fixed metallicity, the scenarios can be discriminated much better in $\mathrm{H} \beta$ than in the metallicity sensitive Fe5335, as expected.

As in the case of colours, index strengths are very similar in scenarios 1,3 , 4 , as well as in scenarios 1b, 3b, 4b, after the end of the last burst (Fig. 2.5). Only scenario 4 , in which the most recent burst has twice the duration (800 Myrs) of those in the other scenarios, differs in $\mathrm{H} \beta$ by more than $0.2 \AA$ from 

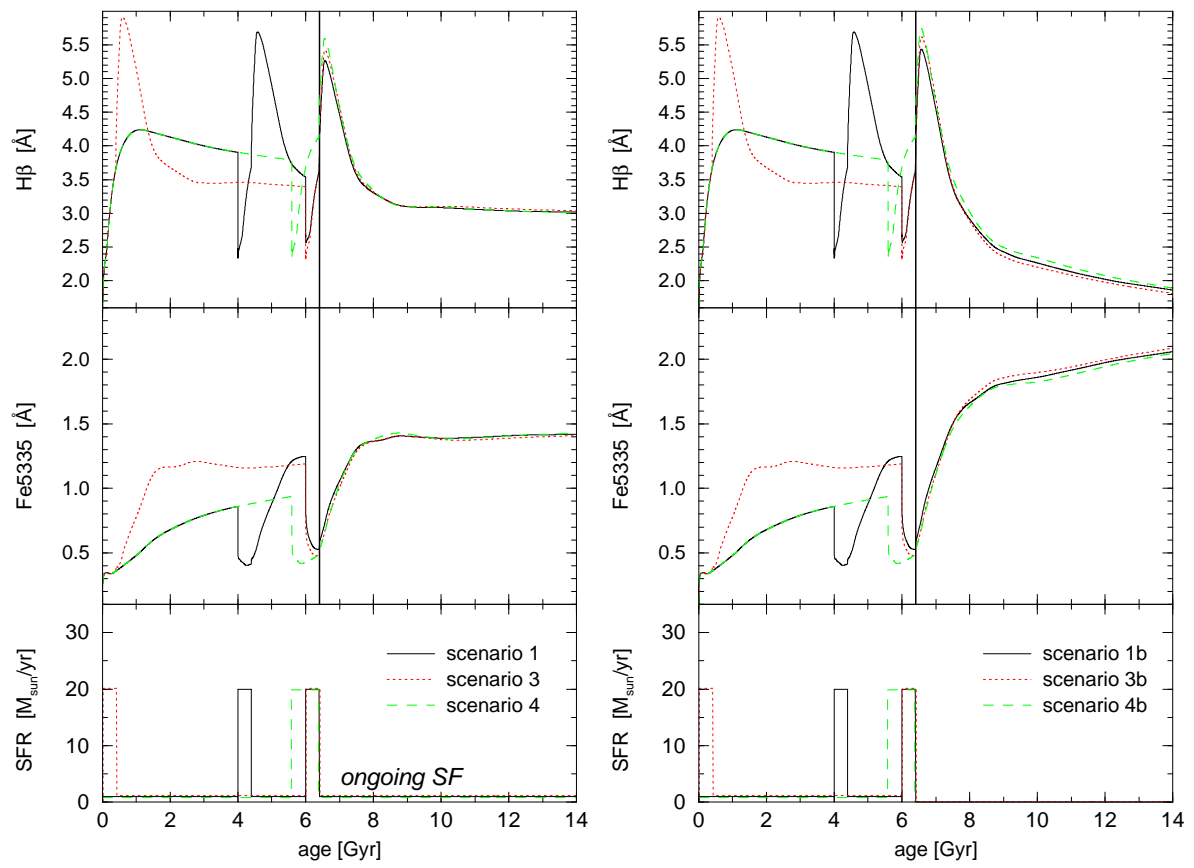

Fig. 2.5: Confrontation of the evolution of scenarios 1, 3, 4 (left) and 1b, 3b, $4 \mathrm{~b}$ (right), respectively, in terms of the Lick indices $\mathrm{H} \beta$ and Fe5335. At $6.4 \mathrm{Gyr}$, when the latest phase of enhanced SF ends, a thick line is plotted. The bottom plots show the respective SFHs to be compared. 

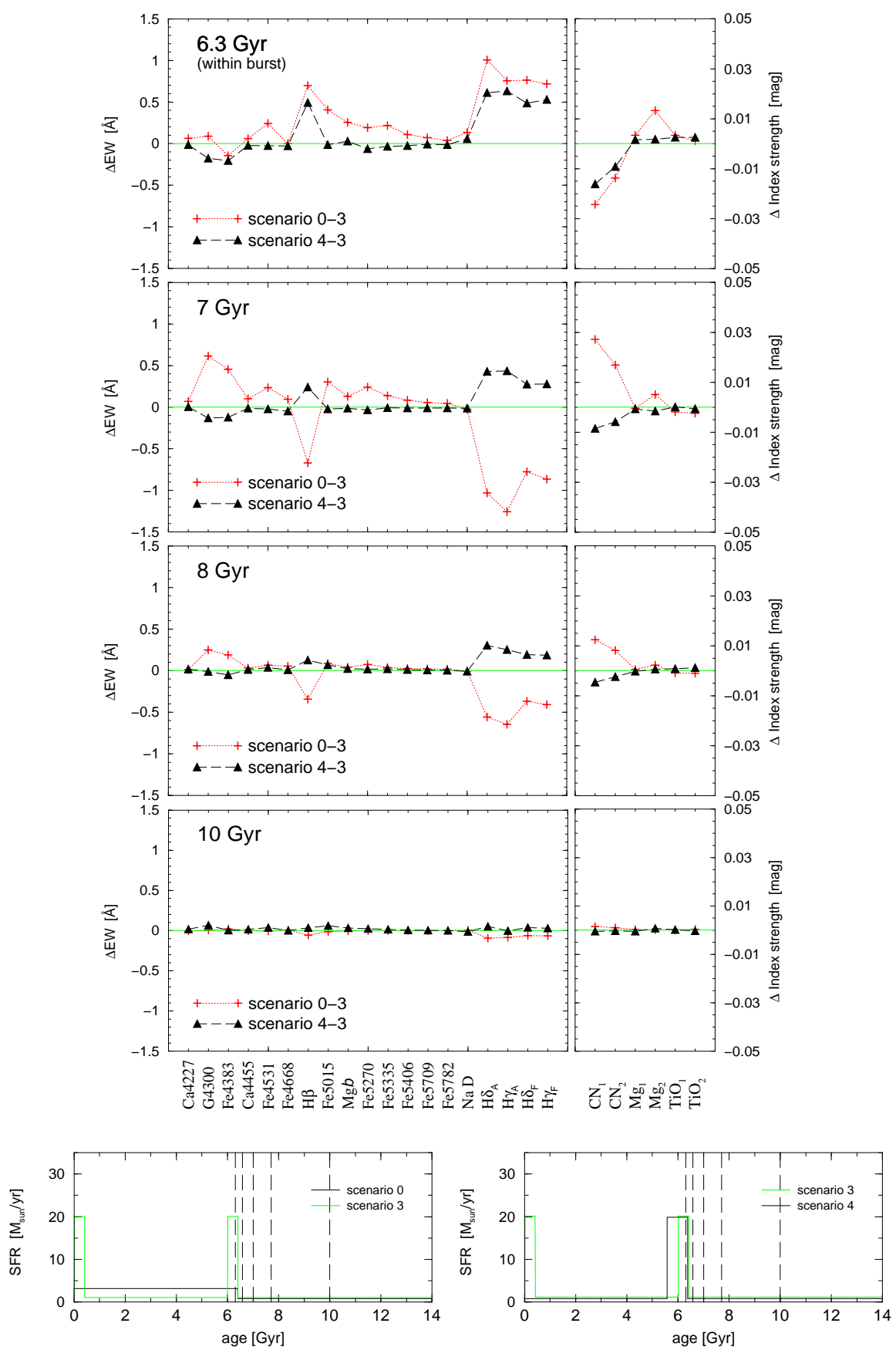

Fig. 2.6: Top: Absolute differences of all 25 Lick indices (in terms of index strength) of the scenarios 0 and 3 and of the scenarios 4 and 3, respectively, for the four model ages indicated in the plot. Bottom: SFHs of the scenarios; the ages $6.3,6.6,7.0,7.7$ and $10 \mathrm{Gyr}$ are indicated by dashed lines. 
the others whithin the first 0.5 Gyr after the end of the most recent burst. This difference in $\mathrm{H} \beta$ is due to the lifetime of early A-type stars of about $\frac{1}{2}$ to $1 \mathrm{Gyr}$, which feature the strongest Balmer lines in their spectra, and which therefore can accumulate during the long duration of the burst in this scenario.

Fig. 2.4 shows that the different bursts of scenarios $0,2,4,6$ can be discriminated much better using indices than using colours. In our simulations, the difference between the strong burst scenario 6 and the non-burst scenario 0 reaches almost $2 \AA$ in $\mathrm{H} \beta$ during the first half Gyr after the end of the burst. About 0.5 Gyr later, the difference has decreased to not more than $0.5 \AA$, but only 2 Gyrs after the end of the burst the difference gets already too small to be measured in praxi. However, if the SF completely stops after the burst (the " $b$ scenarios"), the non-burst scenario can be discriminated from burst-scenarios for about 6 Gyrs in $H \beta$. Only thereafter, this difference decreases to less than $0.2 \AA$.

So far, we have compared the different scenarios only in terms of $\mathrm{H} \beta$ and Fe5335; in Fig. 2.6, we plot the absolute difference in terms of index strength between different scenarios for all Lick indices. For means of clarity, we plot the values for four galaxy ages only $(6.3 \mathrm{Gyr}$, wich is still during the most recent burst, 7 Gyr, 8 Gyr, and 10 Gyr), and for two pairs of scenarios: With scenarios 0 and 3 (crosses in Fig. 2.6) we confront a burst and a non-burst scenario, with scenarios 3 and 4 (triangles in Fig. 2.6) we confront a short burst scenario with a long burst scenario where both bursts form identical amounts of stars in total. (Note that we do not expect the first burst of scenario 3 to have any significant effect on index strengths at the ages considered here; it only guarantees that all scenarios form equal amounts of stars in total.)

¿From these plots, the absolute sensitivity of individual Lick indices to age can directly be read off, showing for example the large age-sensitivity of Balmer lines (note however, that the age-metallicity degeneracy problem is ignored in this approach, since we keep the metallicity constant).

By a galaxy age of $10 \mathrm{Gyr}$, i.e. less than 4 Gyrs after the most recent burst, the scenarios become practically indistinguishable in all indices, with maximal differences of only about $0.1 \AA$. This shows that the results obtained earlier for $\mathrm{H} \beta$ and $\mathrm{Fe} 5335$ are valid for all indices.

We conclude that, compared with broad-band colours, Lick indices do not seriously improve the situation: After a burst, again we are not able to detect any details in the SFH prior to this event, and different burst strengths can be discriminated for a lookback time larger than 2 Gyrs only if the SF completely stops after the latest burst.

\subsubsection{Spectra}

To get an impression of how different scenarios of SF are reflected in the resulting spectra, in Figure 2.7 we plot relative difference spectra for two pairs of scenarios for five evolutionary stages $(6.3,6.6,7.0,7.7$, and $10 \mathrm{Gyr})$. In the left panels, a scenario with a weak, uniform SFR (scenario 0) is confronted with burst scenario 3 ; in the right panels, this burst scenario 3 is confronted with a scenario featuring a burst of equal strength but twice the duration (scenario 4). Note that in both scenarios, SF goes on after the end of the latest burst/the phase of enhanced 

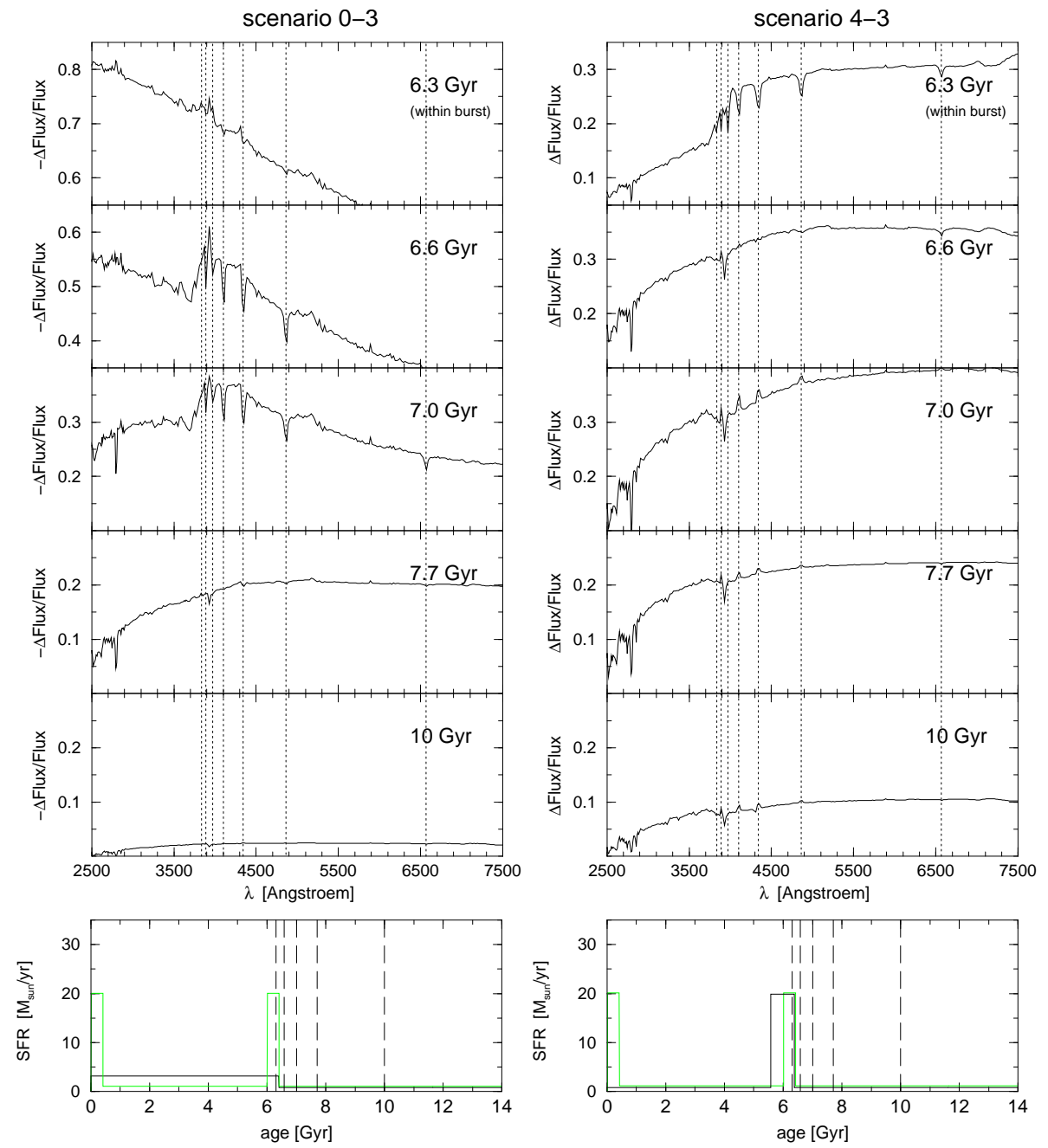

Fig. 2.7: Top: Relative differences of the spectra of the scenarios 0 and 3 (left) and of the scenarios 4 and 3 (right) within the range of the Balmer lines (with markings of the Balmer lines $H_{\alpha}-H_{\eta}$ ) for the five model ages indicated in the plot. Bottom: SFH of the respective scenarios; the ages 6.3, 6.6, 7.0, 7.7 and 10 Gyr are indicated by dashed lines. 
SF. We only plot a small section of our model spectrum from 2500 to $7500 \AA$; for better orientation, Balmer lines $H_{\alpha}$ to $H_{\eta}$ are marked by vertical lines.

In terms of their full spectral energy distributions from $\mathrm{U}$ through $\mathrm{K}$ (not shown here), as for colors and indices, scenarios are nearly indistinguishable 4 Gyrs after the latest burst. However, in contrast to broad-band colors and Lick indices, a clearer distinction between scenarios with bursts of different durations than between bursts of different strength can be observed in the spectral continua, as can clearly be seen in Fig. 2.7.

As expected, and as already shown by using Lick indices, differences in Balmer lines are clearly visible only during a lookback time of about 1 Gyr after the end of the most recent burst (i.e., they disappear at a galaxy age of $7.7 \mathrm{Gyr}$ ); this was already explained by the short lifetimes of stars mainly responsible for these spectral lines. Other absorption lines easily visible in the plots are the MgII line (at $\lambda=2798.00 \AA$ ) and CaII $\mathrm{K}$ (at $\lambda=3933.44 \AA$, between $\mathrm{H}_{\epsilon}$ and $\mathrm{H}_{\zeta}$ ). At fixed metallicity, the MgII line is most pronounced in the spectra of early F-type stars with a lifetime of approximately 2 Gyrs. The CaII K line, on the other hand, is strongest in the spectra of late F-type and early G-type stars; these stars have lifetimes of 2-6 Gyrs. These lifetimes explain the relatively complicated behaviour of the lines in the relative difference spectra.

For example, at a galaxy age of 6.6 Gyr (300 Myrs after the end of the last burst), CaII $\mathrm{K}$ is less deep in burst scenario 3 than in non-burst scenario 0 (Fig. 2.7, left panels); about 1 Gyr later, at a galaxy age of $7.7 \mathrm{Gyr}$, the line is slightly stronger in the burst scenario: Shortly after the end of the epoch of enhanced SFR more G-type stars have been accumulated in the non-burst scenario 0; stars of this type originating from the early burst of scenario 3 are not alive any more at this time. 1 Gyr later, late F- and early G-type stars originating from the most recent burst of scenario 3 outweight the respective stars accumulated in the non-burst scenario.

The model spectra we use have a resolution too low to analyse spectral features in more detail. However, due to the very different lifetimes of stars of various types which are responsible for different spectral lines, and due to what can be seen already in our low-resolution spectra, we expect that highresolution spectra can reveal much more precise information about the recent $\mathrm{SFH}$ of galaxies than colors or the classical set of Lick indices. A new set of spectral indices with narrower passband definitions could provide such a tool. It requires large telescopes both for the calibrations on stellar spectra and for galaxy observations. It will, therefore, still be limited to reasonable small distances.

We conclude that - with the possible exception of high-resolution spectroscopy not studied here - intermediate-resolution spectroscopy does not allow to significantly improve upon the details, nor upon the lookback times to which SFHs of galaxies can be retrieved as compared to multi-band photometry

\subsubsection{Some remarks on CMDs and integrated light}

Before we turned to study how far back in time and to what accuracy more complex SFHs can be discriminated on the basis of CMDs, compared to integrated light, we have computed a large grid of single stellar population (SSP) models 

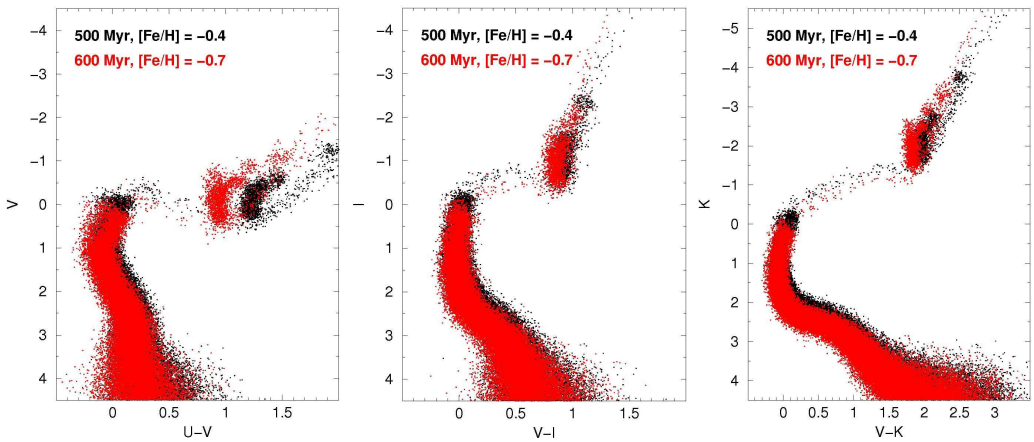

(a) SSPs with age metallicity combinations $(500 \mathrm{Myr},[\mathrm{Fe} / \mathrm{H}]=-0.4)$ and $(600 \mathrm{Myr},[\mathrm{Fe} / \mathrm{H}]=-0.7)$. Both SSPs can hardly be distinguished in $\mathrm{V}-\mathrm{I}$ and $\mathrm{V}-\mathrm{K}$, but clearly split up in $\mathrm{U}-\mathrm{V}$.
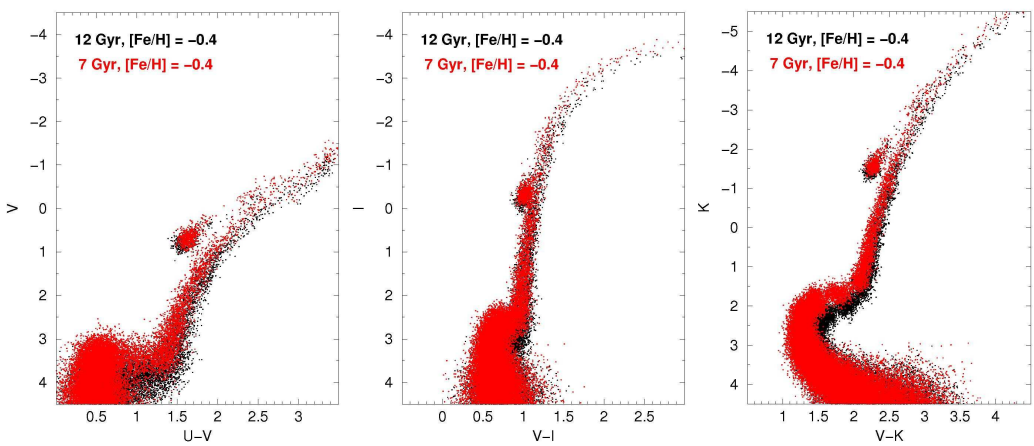

(b) SSPs with age metallicity combinations $(12 \mathrm{Gyr},[\mathrm{Fe} / \mathrm{H}]=-0.4)$ and $(7 \mathrm{Gyr}$, $[\mathrm{Fe} / \mathrm{H}]=-0.4)$. The SSPs can hardly be distinguished in all three colours, best in $\mathrm{V}-\mathrm{K}$ at the main sequence turn-off point.
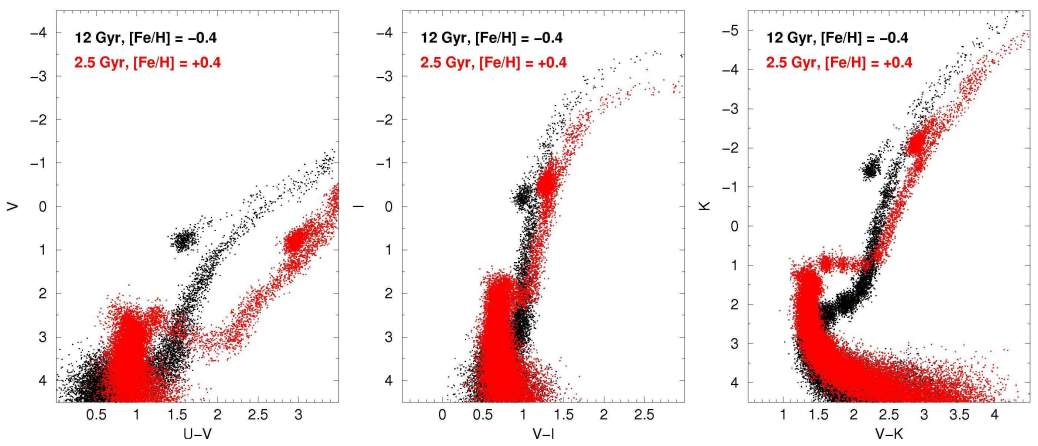

(c) SSP with age metallicity combinations $(12 \mathrm{Gyr},[\mathrm{Fe} / \mathrm{H}]=-0.4)$ and $(2.5 \mathrm{Gyr}$, $[\mathrm{Fe} / \mathrm{H}]=+0.4)$. All SSPs clearly split up in all three colours.

Fig. 2.8: Comparision of CMDs in U-V, V-I, and V-K for three pairs of SSP models with different age metallicity combinations: Two young SSPs (a), two old SSPs (b), and an old and an intermediate age SSP (c). 
Table 2.2: Integrated colours in U-V, V-I, V-K for SSP models with different age metallicity combinations, arranged in three pairs with equal or very similar colour in $\mathrm{V}-\mathrm{I}$

\begin{tabular}{ccccc}
\hline \hline age & {$[\mathrm{Fe} / \mathrm{H}]$} & $\mathrm{U}-\mathrm{V}[\mathrm{mag}]$ & $\mathrm{V}-\mathrm{I}[\mathrm{mag}]$ & $\mathrm{V}-\mathrm{K}[\mathrm{mag}]$ \\
\hline $500 \mathrm{Myr}$ & $-0.4 \mathrm{dex}$ & 0.4 & 0.7 & 2.5 \\
$600 \mathrm{Myr}$ & $-0.7 \mathrm{dex}$ & 0.4 & 0.7 & 2.3 \\
$12 \mathrm{Gyr}$ & $-0.4 \mathrm{dex}$ & 1.4 & 1.2 & 3.1 \\
$7 \mathrm{Gyr}$ & $-0.4 \mathrm{dex}$ & 1.2 & 1.2 & 3.1 \\
$12 \mathrm{Gyr}$ & $-0.4 \mathrm{dex}$ & 1.4 & 1.2 & 3.1 \\
$2.5 \mathrm{Gyr}$ & $+0.4 \mathrm{dex}$ & 1.5 & 1.3 & 3.8 \\
\hline
\end{tabular}

with different metallicities in their time evolution and identified a number of very different age-metallicity combinations that - due to the age-metallicity degeneracy - have the same or very similar V-I. In Fig. 2.8, we confront some of these pairs in terms of CMDs in various passband combinations. Colours and age-metallicity combinations of all models are given in Tab. 2.2.

Fig. 2.8 (a) shows a comparison of CMDs in different passband combinations for two SSPs with age metallicity combinations $(500 \mathrm{Myr},[\mathrm{Fe} / \mathrm{H}]=-0.4)$ and $(600 \mathrm{Myr},[\mathrm{Fe} / \mathrm{H}]=-0.7)$. These combinations of relatively young clusters are practically indistinguishable in $\mathrm{V}-\mathrm{I}$ and $\mathrm{V}-\mathrm{K}$, but clearly separate in $\mathrm{U}-\mathrm{V}$. In terms of integrated colours, on the other hand, these SSPs are indistinguishable in $\mathrm{U}-\mathrm{V}$ and $\mathrm{V}-\mathrm{I}$, but differ slightly in $\mathrm{V}-\mathrm{K}$ since this colour is mainly determined by luminous giant stars which differ slightly in position in the V-K CMD.

Fig. 2.8 (b) shows CMDs for two old SSPs with age metallicity combinations $(12 \mathrm{Gyr},[\mathrm{Fe} / \mathrm{H}]=-0.4)$ and $(7 \mathrm{Gyr},[\mathrm{Fe} / \mathrm{H}]=-0.4)$. In this case, the CMDs are indistinguishable both in $\mathrm{U}-\mathrm{V}$ and $\mathrm{V}-\mathrm{I}$, but can slightly be distinguished in $\mathrm{V}-\mathrm{K}$ via the luminosity of the main sequence turn-off point. Again, in terms of integrated colours the situation is complementary: Here, both SSPs exhibit similar colours in both $\mathrm{V}-\mathrm{I}$ and $\mathrm{V}-\mathrm{K}$, but slightly split up in $\mathrm{U}-\mathrm{V}$.

In Fig. 2.8 (c) we show a comparison of CMDs for an intermediate age metal-rich and an old SSP poorer in metallicity with age metallicity combinations $(2.5 \mathrm{Gyr},[\mathrm{Fe} / \mathrm{H}]=+0.4)$ and $(12 \mathrm{Gyr},[\mathrm{Fe} / \mathrm{H}]=-0.4)$. All model CMDs can clearly be distinguished. However, in terms of inegrated colours only in $\mathrm{V}-\mathrm{K}$ the SSPs clearly split up, whereas in both $\mathrm{U}-\mathrm{V}$ and $\mathrm{V}-\mathrm{I}$ the SSPs can hardly be distinguished.

We conclude that for young stellar populations the U-band is important for age determinations and for intermediate-age and old ( $>3 \mathrm{Gyr}$ ) populations the K-band is important for metallicity determination. We also find that CMDs and integrated light colours give complementary information: In terms of CMDs young stellar populations can best be discriminated using $\mathrm{U}-\mathrm{V}$, and old populations only if NIR observations are available. In terms of integrated colours, $\mathrm{V}-\mathrm{K}$ can be particularly useful to discriminate young populations, and $\mathrm{U}-\mathrm{V}$ to discriminate older ones (cf. Sect. 2.3.1, Fig. 2.1). In all cases, however, a long wavelength basis is essential (ideally UV or U-band through NIR). 

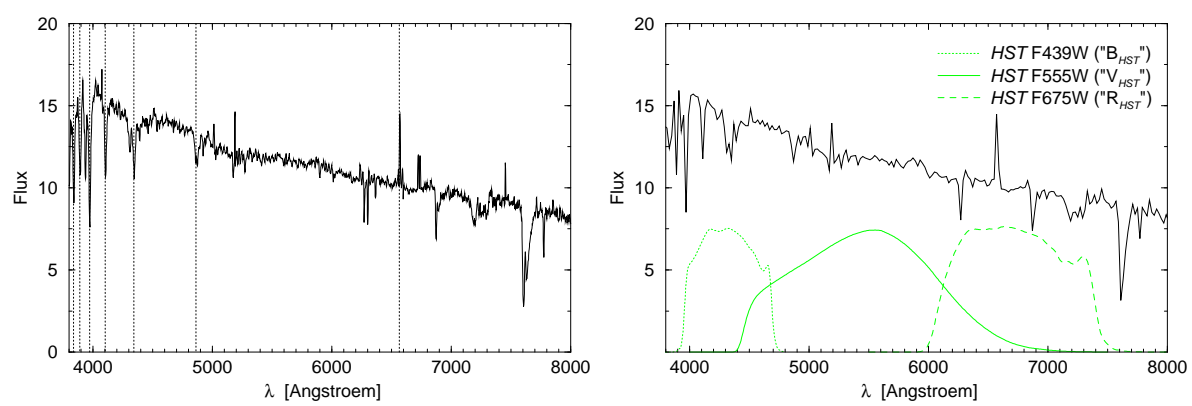

Fig. 2.9: Integrated-light spectrum of the LMC bar field (dereddened). Left: Original spectrum. Right: Spectrum with lowered resolution to be compared with model spectra, and the three filters used for the analysis.

Table 2.3: Colours derived from the integrated-light spectrum of the LMC bar field, obtained by folding the spectrum with the respective filter functions.

\begin{tabular}{ccc}
\hline \hline$(\mathrm{B}-\mathrm{V})_{H S T}$ & $(\mathrm{~V}-\mathrm{R})_{H S T}$ & $(\mathrm{~B}-\mathrm{R})_{H S T}$ \\
\hline $0.44 \mathrm{mag}$ & $0.53 \mathrm{mag}$ & $0.97 \mathrm{mag}$ \\
\hline
\end{tabular}

\subsection{The LMC bar field and its spectrum}

To compare, on a practical example, how far back in time and to what accuracy the SFH can be reconstructed, we compare a deep HST-based CMD analysis (Smecker-Hane et al. 2002) with an analysis of the integrated spectrum of exactly the same star field in the LMC obtained trailing a slit exactly across the region for which the deep HST CMD was obtained. However, we want to emphasize that we do not try to find the real physical SFH of the LMC. Like in the previous sections, our interest is purely methodological and our aim is to contrast the power and limitations of both approaches.

The integrated-light spectrum of the region in the LMC bar (FoV: 2.5' $\left.\times 5^{\prime}\right)$ was obtained at the ESO 3.6m telescope on La Silla in Dezember 2000 by E. Pompei and D. Alloin. The 2000 coordinates of the field are: $\alpha=05: 23: 17$ and $\delta=-69: 45: 42$; for observational details cf. Alloin et al. (2002). The original spectrum has been dereddened using Cardelli et al.'s (1989) extinction law, with $\mathrm{E}(\mathrm{B}-\mathrm{V})=0.075 \mathrm{mag}$ (corresponding $\mathrm{A}_{B}=0.324$ and $\mathrm{A}_{V}=0.249$, respectively) taken from Schlegel et al. (1998).

Fig. 2.9 (left) shows the resulting spectrum, Fig. 2.9 (right) the same spectrum but with lowered resolution to be compared with our model spectra. For better orientation, the Balmer lines $H_{\alpha}$ to $H_{\eta}$ are marked by vertical lines in the left panel.

Unfortunately, the model spectra we use have a resolution too low to successfully analyse spectral features; therefore, our analysis mainly depends on broad band colours obtained from the spectrum, and the shape of the spectral energy distribution.

The usable wavelength range of the spectrum allows for folding with three 


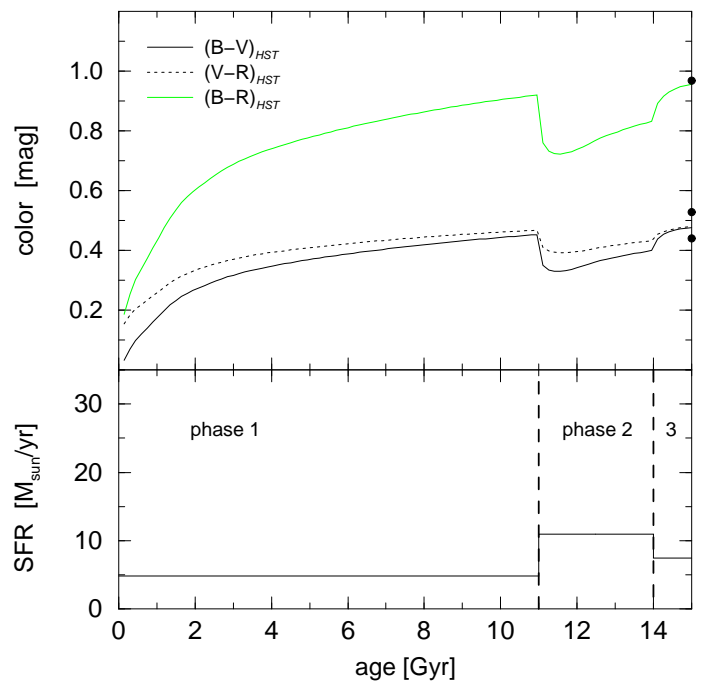

Fig. 2.10: Photometric evolution of a simple 3-phase scenario in terms of (B$\mathrm{V})_{H S T},(\mathrm{~V}-\mathrm{R})_{H S T}$, and $(\mathrm{B}-\mathrm{R})_{H S T}$ with the corresponding $\mathrm{SFH}$; the observed LMC bar field colours (cf. Table 2.3) are marked with black dots at 15 Gyr galaxy age.

filters (filter functions shown in Fig. 2.9, right panel): $H S T$ WFPC2 F439W, $\mathrm{F} 555 \mathrm{~W}$, and $\mathrm{F} 675 \mathrm{~W}$ (in the following referred to as $\mathrm{B}_{H S T}, \mathrm{~V}_{H S T}, \mathrm{R}_{H S T}$ ); Table 2.3 gives the resulting colours obtained by folding the spectrum with the respective filter functions in the Vegamag system.

\subsubsection{A simple 3-phase SFH}

In the previous section, we have shown that, using integrated light only, variations in the SFH of a galaxy can be traced for only about 1 Gyr, at the utmost 4 Gyrs, of lookback time, unless the SF is completely truncated at some point of galactic evolution which is definitely not the case for the LMC. This means, the relative distribution of SF within early epochs ( $\geq 4$ Gyr ago) of galaxy evolution is almost irrelevant, and that within the more recent history ( $4 \mathrm{Gyr} \geq$ lookback time $\geq 1$ Gyr) only of weak relevance for the observed colours. Calculating and analysing a much larger grid of SF scenarios than just the few particularly instructive examples presented here in all optical and NIR colours has revealed that three different epochs can bee identified in the SFH of all galaxy models for which the relative amounts of stars formed in each of them clearly defines the galactic colours. These three phases are:

1. From the onset of SF until about $4 \mathrm{Gyr}$ ago

2. Between 4 Gyr and 1 Gyr ago

3. The last (= most recent) $1 \mathrm{Gyr}$

I.e., the relative distribution of SF between these 3 phases is important and not only the SF within the last Gyr. Therefore, it should be possible to 


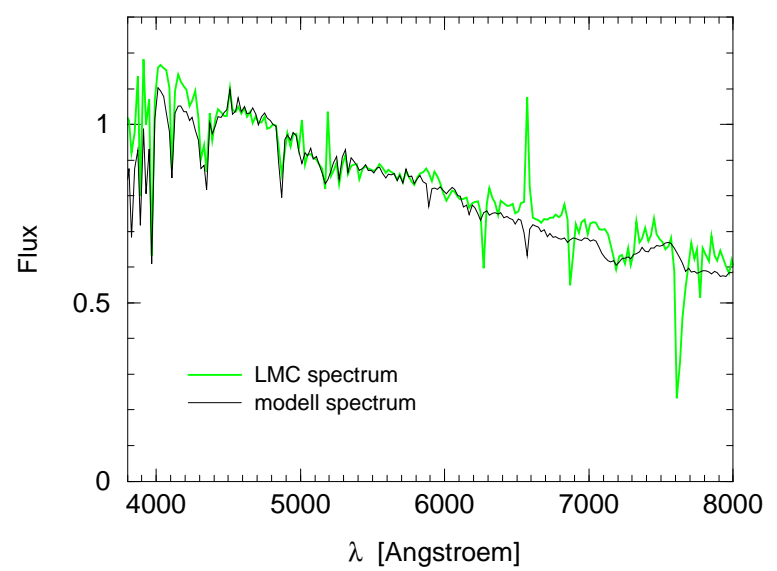

Fig. 2.11: Model spectrum of the 3-phase scenario (cf. Fig. 2.10) at a galaxy age of 15 Gyr against the observed LMC spectrum. Both spectra are normalized at $4810 \AA$ (arbitrary value).

analyse any given set of colours (or, as we have also learned at the study, even Lick indices) in terms of the relative contributions from only 3 different phases of SF, if the phases are chosen appropriately ${ }^{2}$ :

Figures 2.10 and 2.11 show that this simple "3-phase scenario" can indeed fit both the observed colours (which are marked with black dots at the right edge of Fig. 2.10, top panel) and the observed spectrum (cf. Fig. 2.11) of the LMC bar field reasonably well; differences still visible are most likely due to our disregarding of any chemical enrichment history and the fact that we assume a constant SFR within phase 3.

Fig. 2.12 illustrates the influence of the 3 different epochs of SF by showing, for the SFH given above, the spectral contributions of stellar populations originating from each of these phases to the total spectrum after 15 Gyrs: The upper panel shows, e.g., that phase 1, though its duration is almost three times longer than that of phase 2, contributes only about half of the light of phase 2 to the total spectrum. The lower panel, on the other hand, shows that the first 14 Gyrs together have roughly the same influence on the final shape of the spectrum as the most recent 1 Gyr of galaxy evolution.

\subsubsection{Some experimentation}

We now systematically vary the SFH within each of the 3 phases of our simple 3-phase scenario to study in how far this affects our ability to track back SFH details as a function of lookback time.

In the six scenarios shown in Fig. 2.13, the constant SF within the 3 phases is, for each phase, replaced by a burst-like SF at the end and the beginning of

\footnotetext{
${ }^{2}$ In order to compare our results with a CMD-based SFH obtained for the same star field (cp. Sec. 2.4.3), we assume a total galaxy age of 15 Gyr.
} 

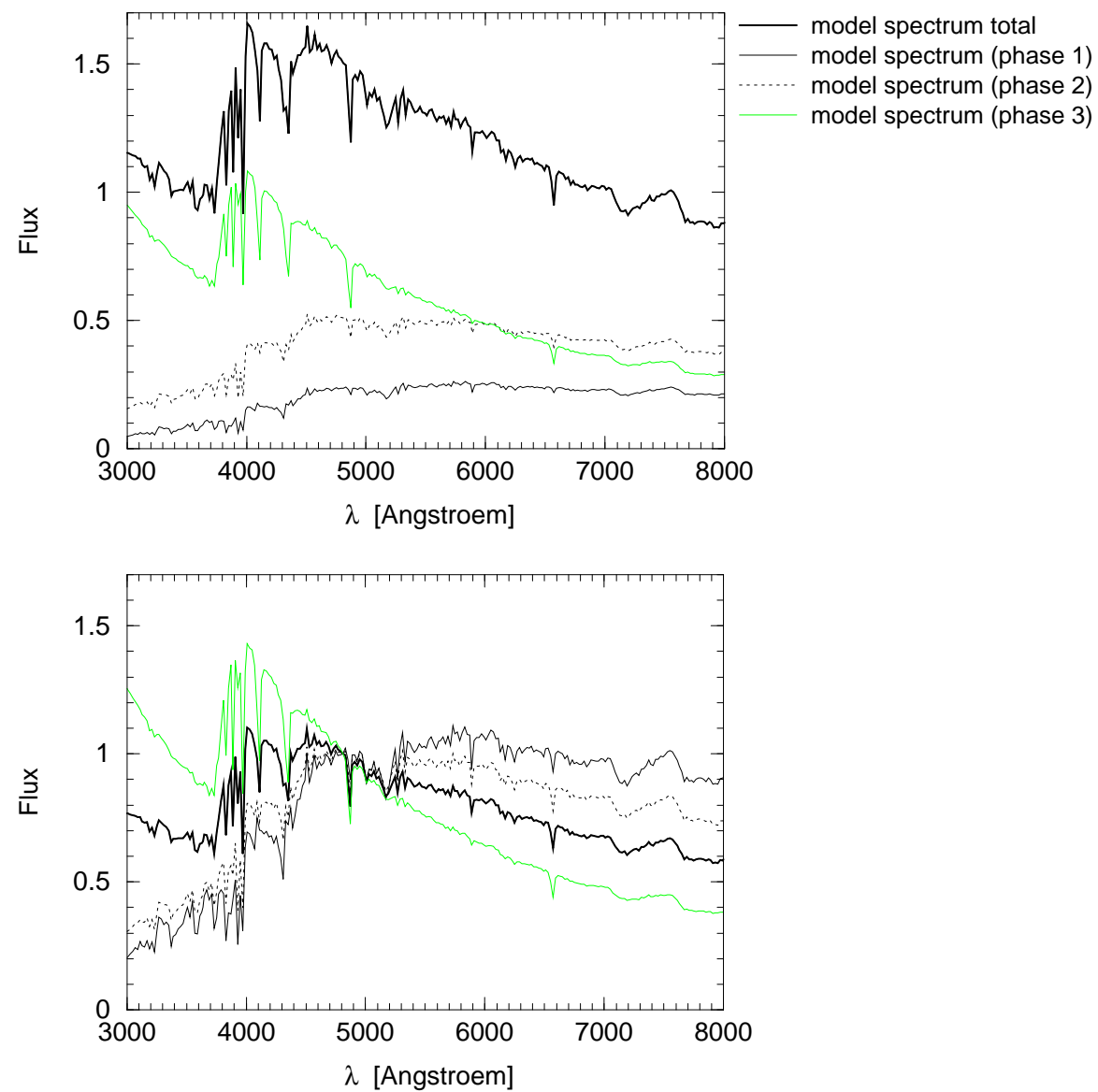

Fig. 2.12: Contribution of the 3 phases of the 3 -phase scenario to the total spectrum. Top: Absolute contribution (i.e., summation of the 3 subpopulation spectra gives the total spectrum). Bottom: Subpopulation spectra and total spectrum normalized at $4810 \AA$ (arbitrary value).

the phase, respectively. We find that this kind of variations has almost no effect on the final colours $\left(\Delta\right.$ colour $<0.05$ mag even in $\left.(\mathrm{B}-\mathrm{R})_{H S T}\right)$ if applied to phase 1 or 2 , because the relative distribution of SF between the 3 phases remains unchanged. Remarkably, in our example it is not even possible to decide if the galaxy is 15 or 6 Gyrs old (cf. scenario $a$ and $b$ ). The same kind of variation applied to phase 3 (i.e., to the most recent 1 Gyr of galaxy evolution), however, results in a significant change of colour $\left(\Delta(\mathrm{B}-\mathrm{R})_{H S T} \approx 0.2 \mathrm{mag}\right)$.

In the scenarios shown in Fig. 2.14, the $\mathrm{SF}$ within the 3 phases remains constant, but, compared to the original scenario, with half and twice the star formation rate (SFR), respectively. As before, the variation is applied to each of the phases. Since this kind of variation changes the relative distribution of SF between the 3 phases, the effect on the final colours after 15 Gyrs is much larger than before, if applied to phases 1 and $2\left(\Delta\right.$ colour $\approx 0.1 \mathrm{mag}$ in $\left.(\mathrm{B}-\mathrm{R})_{H S T}\right)$. Variation of phase 3 (scenarios $k, l$ ) has a similar effect on colours $\left(\Delta(\mathrm{B}-\mathrm{R})_{H S T}\right.$ 
$\approx 0.2 \mathrm{mag})$ as the kind of variation applied in Fig. 2.13 (scenarios $e, f)$.

The experiments sketched above confirm the expectations from our previous work (cf. Sect. 2.3). In phase 1 and 2, changes of the total mass of stars produced within the phase have a stronger effect on colours than changes in the mere distribution of SF within the phase. A similar behaviour for the effects of SFR changes in phases 1-3 is found in the spectral energy distribution (not shown here). We conclude:

1. The SFH of a galaxy can be satisfactorily reconstructed in terms of three main phases only, corresponding to 1 Gyr lockback time (phase 3), 3-4 Gyr lookback time (phase 2), and more than 4 Gyr lookback time (phase $1)$.

2. The relative amounts of stars formed in each of the three phases can well be determined.

3. The distribution of SF within phases 1 and 2 (the earliest two phases) cannot be recovered.

4. Only within the last $\sim 1$ Gyr can some SFH details be recovered.

\subsubsection{The CMD based SFH}

The resolved stellar population of the LMC bar field was observed with HST WFPC2 in 1997 (PI: Smecker-Hane).

Smecker-Hane et al. (2002) presented a SFH derived from their analysis of the deep CMD obtained from these observations; Fig. 2.15 (left) shows their CMD together with their published $\mathrm{SFH}^{3}$; note that, before doing any analysis of the data, they converted their originally observed HST F555W and F814W magnitudes to Johnson V and Kron-Cousins I magnitudes. The right panel of the same figure shows a CMD at a galaxy age of 15 Gyr calculated with our GALEV models, using the SFH from Smecker-Hane et al. (2002). We colourcoded 4 epochs of star formation.

Figures 2.16 and 2.17, left panels, show the spectrophotometric evolution and the model CMD at 15 Gyr galaxy age resulting from Smecker-Hane et al.'s SFH. The middle panels of the same figures show the same for a scenario using a simplified version of this SFH. "Simplified" means that within each of the four epochs of SF (colour coded in Fig. 2.15), the SFR is put to a constant value, conserving the number of stars produced in this epoch (i.e., without changing the relative amounts of SFR between the 4 epochs). Note, however, that the latter is not the case when compared to the 3-phase SFH (right panels in Figs. 2.16 and 2.17).

In the next section, both Smecker-Hane et al.'s original and the simplified 4-phase scenario will be confronted against each other and against the simple 3-phase scenario.

\footnotetext{
${ }^{3}$ Note that, in this plot, we have inverted their time axis to match the standard used in our paper (evolution of the galaxy from left to right).
} 

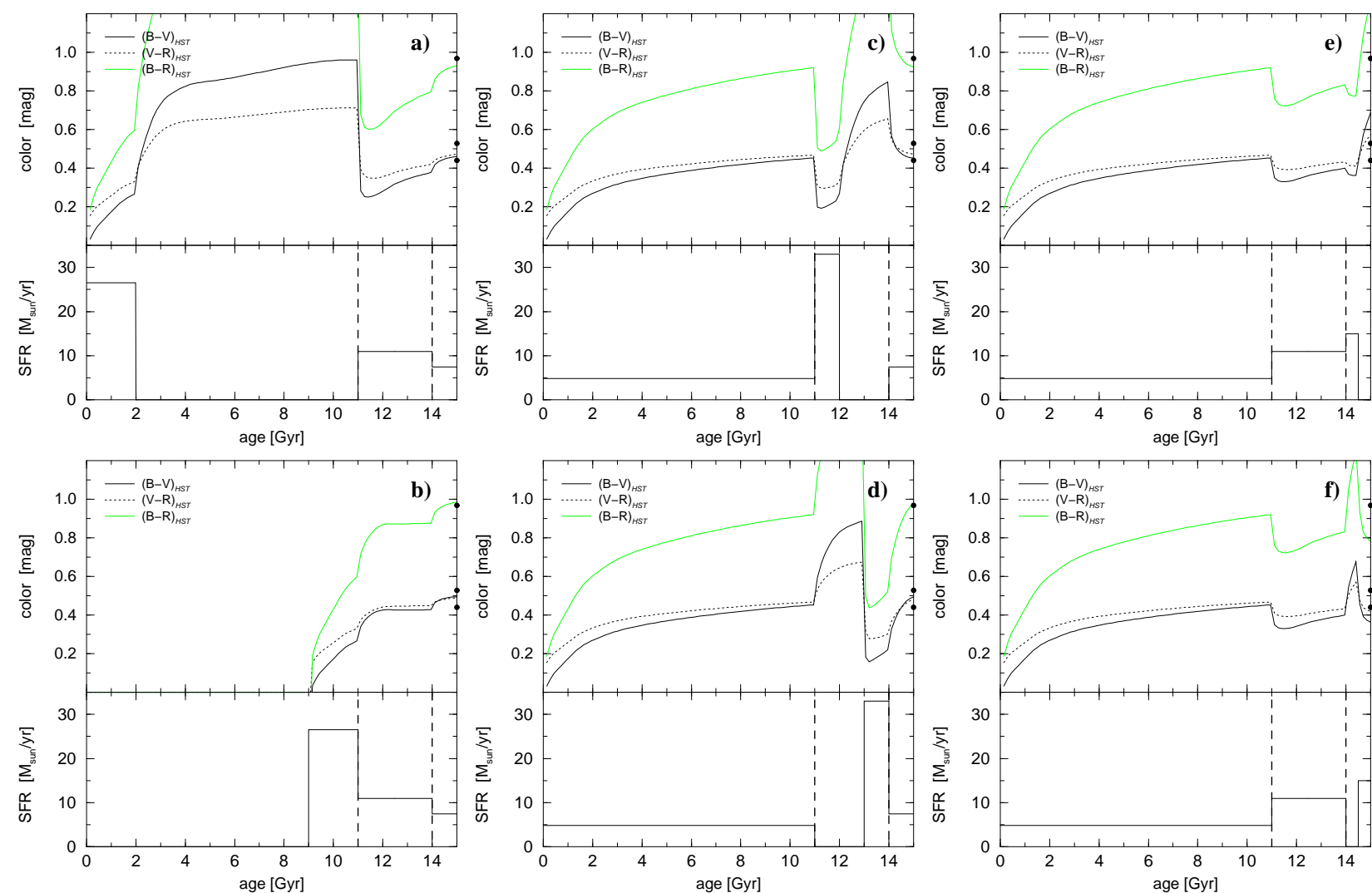

Fig. 2.13: Variations of the 3-phase scenario (cf. Fig. 2.10): Scenarios with systematic variations of the SFH within phase 1 ( $a, b)$, phase $2(c, d)$, and phase $3(e, f)$; note that the relative distribution of the total amount of SF between the 3 phases remains unchanged. 

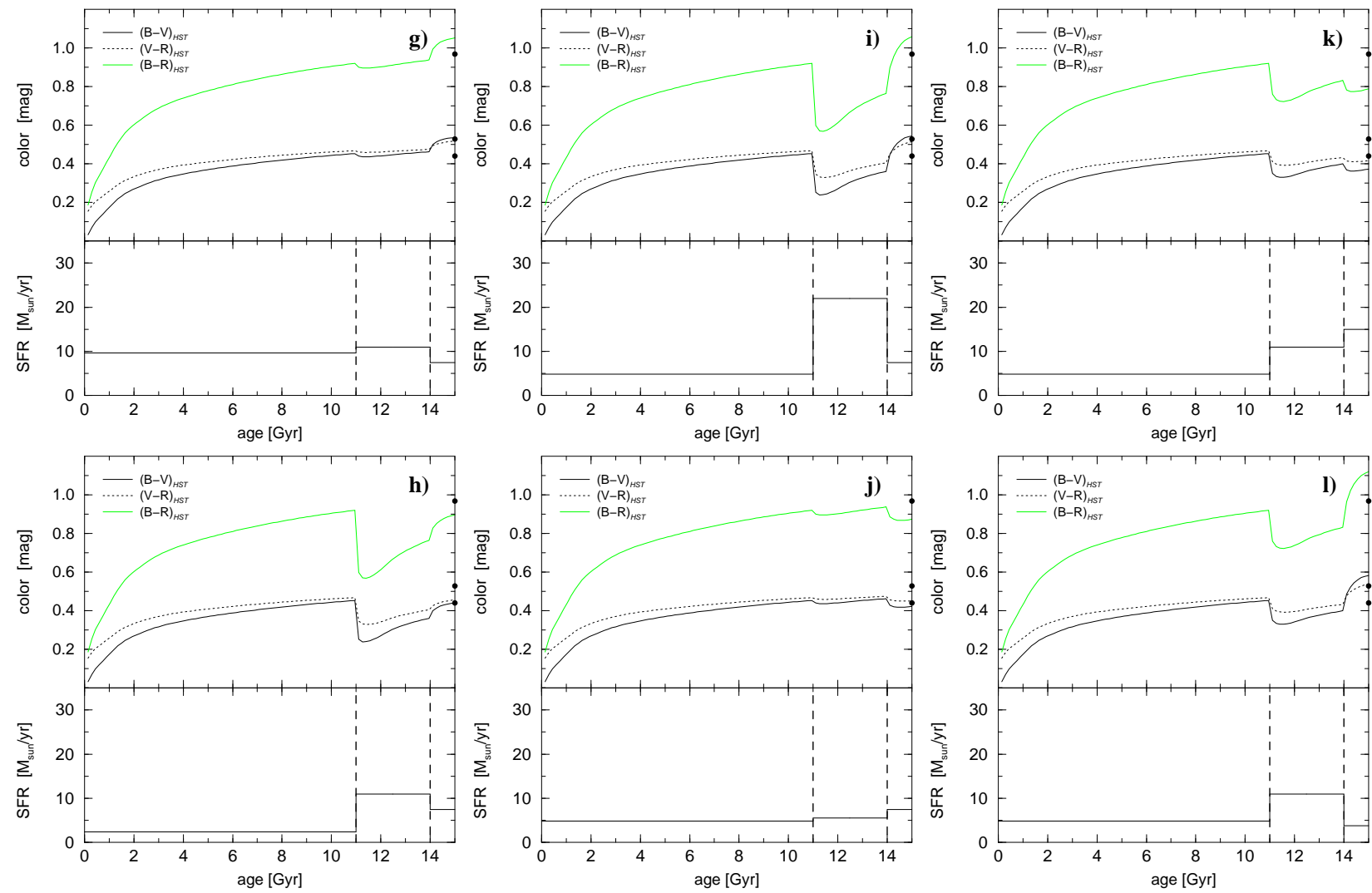

Fig. 2.14: Variations of the 3-phase scenario (cf. Fig. 2.10): Scenarios with twice and half the SF, respectively, within phase 1 ( $g$, $h$ ), phase $2(i, j)$, and phase $3(k, l)$; the relative distribution of the total amount of SF between the 3 phases is changed. 


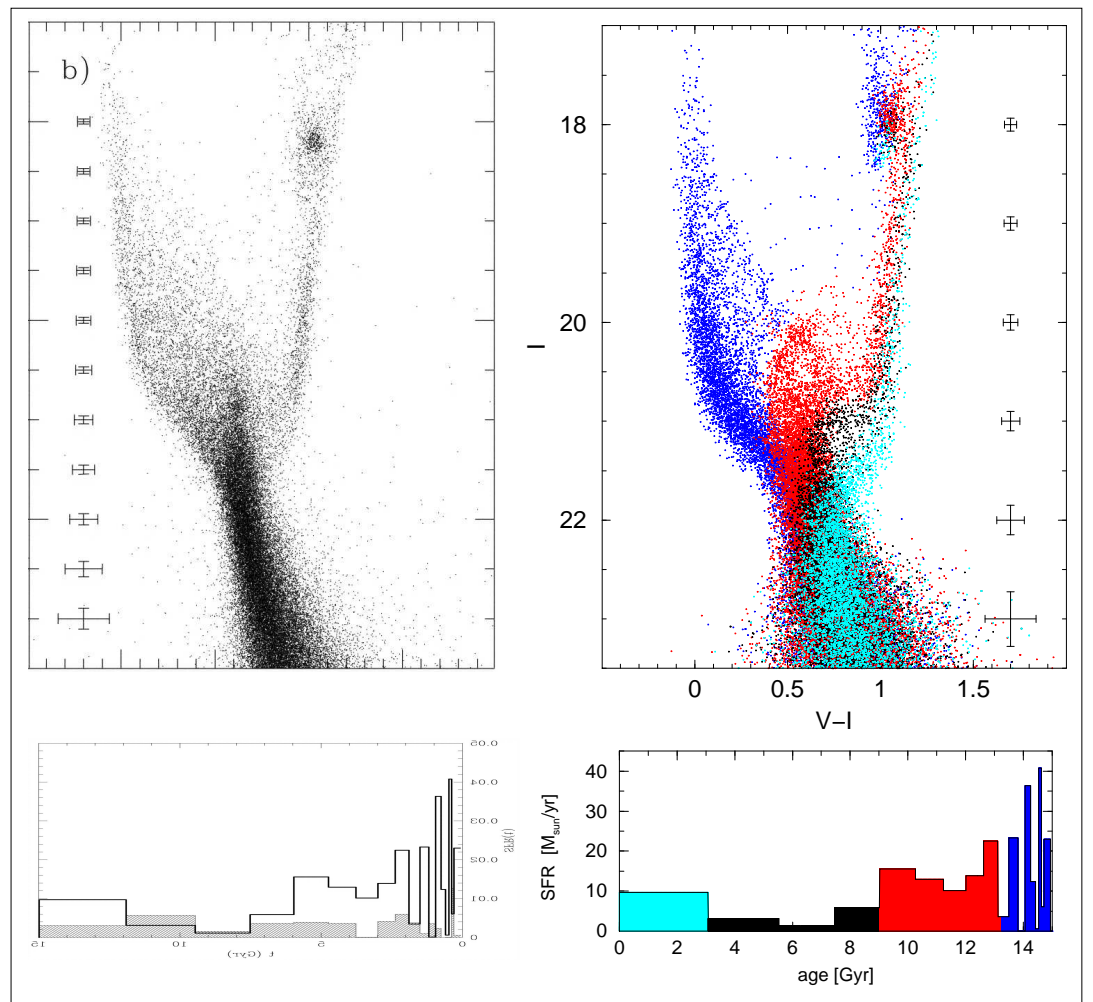

Fig. 2.15: Left: CMD and SFH as presented by Smecker-Hane et al. (2002). Right: Model CMD at a simulated galaxy age of 15 Gyr, using Smecker-Hane et al.'s SFH. Stellar populations originating from 4 different phases of star formation are coded in different colours (cf. the electronic version of this paper).

\subsubsection{CMDs and integrated light: Comparison and Con- clusions}

Figures 2.16 and 2.17 confront the spectrophotometric evolution and the CMDs, respectively, of three scenarios using Smecker-Hane et al.'s (2002) original SFH (left panels), the simplified Smecker-Hane SFH (central panels), and the "simple 3-phase" SFH (right panels).

In terms of colours and spectra, the scenario using Smecker-Hane et al.'s original SFH is, at a galaxy age of 15 Gyr, practically identical to the 3-phase scenario; both differ slightly $\left(\Delta(\mathrm{B}-\mathrm{R})_{H S T}<0.1 \mathrm{mag}\right)$ from the simplified SmeckerHane scenario.

This shows that two scenarios with very different SFHs can result in very similar, observationally indistinguishable integrated-light properties; on the other hand, scenarios with very similar "global" SFH (as e.g. the original and simplified Smecker-Hane et al. SFH) can differ in their final colours. The results show not only the ambiguity of SFHs obtained from integrated light but also emphasize again the importance of the most recent (i.e., lookback time $\leq 1$ Gyr) 
SF for the observed optical colours.

In terms of CMDs, the original and simplified Smecker-Hane et al. scenarios seem to be very similar; the CMD computed using the 3-phase scenario differs from both: A careful visual examination of the plots reveals that in the first two scenarios, the "gap" in their SF between 3 and 9 Gyr galaxy age transforms into an area of less density in the corresponding CMDs around the main sequence turn-off (cf. the black dots in Fig. 2.15, right panel), as expected, wheras in case of the 3-phase scenario, which lacks this "gap" in its SFH, the density is higher in the same area of the CMD. If this result can be validated by a solid numerical comparison between the CMDs (beyond the scope of the present paper), this means that the global distribution of SF is crucial for the appearance of CMDs.

However, note that not even CMDs are free of ambiguity. The ambiguity in the SFH derived from a CMD increases with increasing lookback time, as was the case for integrated-light-derived SFHs. SFHs like that presented by SmeckerHane et al. (2002) are most likely not as exact as their complicated shape suggests; this was already shown by Lilly (2003) and most recently confirmed by the "double-blind Cozumel experiment" (cf. Holtzman 2005).

\subsection{Summary and Conclusions}

In this study, we explored to what precision and how far back in time star formation histories can be determined using integrated colours, lick indices, spectra or CMDs. In our methodological study we compared different kinds of simplified SFHs, i.e. epochs of constant low and high SFRs and investigated how much can be revealed by integrated galaxy data about the SFH before the most recent burst or epoch of important SF. We summarise our results as follows:

Optical colours mainly depend on the amount of blue luminous high-mass stars that formed within the last $0.5-1 \mathrm{Gyr}$, and their relation to the amount of red low-mass stars formed in earlier epochs of star formation. On the basis of colours, different SF scenarios can at best be discriminated for $1-4$ Gyr after the end of SF, and there is absolutely no way to reveal the SFH prior to a burst or the last major epoch of SF.

On the basis of Lick indices, different SF scenarios can again be discriminated for maximally $1-4$ Gyr after the end of SF (using $\mathrm{H} \beta$, e.g.). Age-sensitive lick indices allow for longer lookback times with much difference in index strength between different scenarios; 4 Gyrs after the end of the latest burst, however, all scenarios are, in practice, indistinguishable in all indices. Remarkably, this is the same maximal lookback time as for broad-band colours.

On the basis of low/intermediate resolution spectroscopy, there is again no way to reveal the SFH prior to a burst or the last major epoch of SF, and different scenarios can not be discriminated any more after $1-4$ Gyr. High resolution high $\mathrm{S} / \mathrm{N}$ spectroscopy and narrow band indices will do somewhat better with e.g. MgII2798 and CaIIK3933 being visible for $\sim 2$ and $2-6$ Gyr, respectively (cf. also Bruzual 2007). 

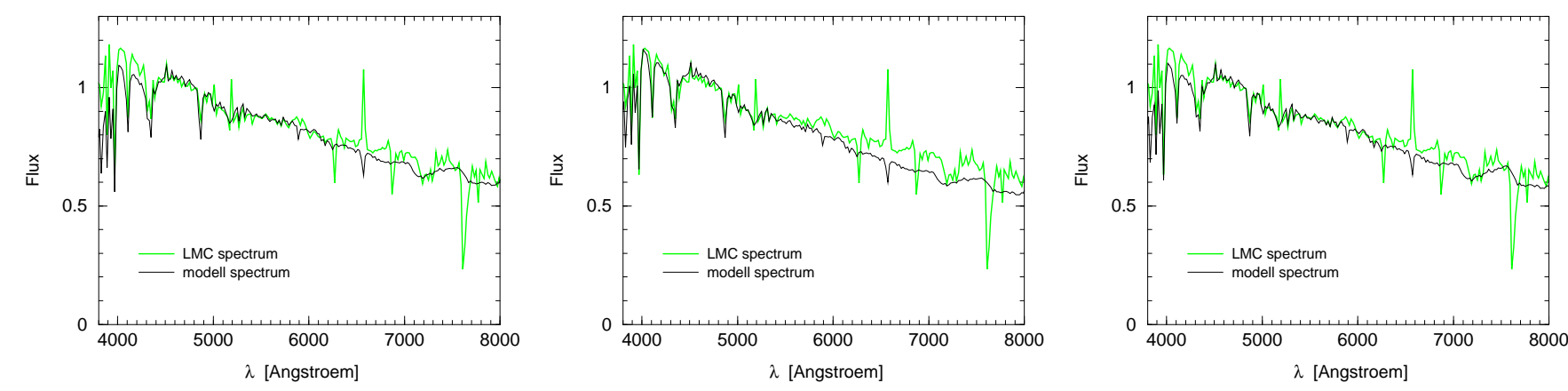

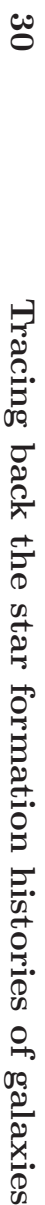

(central panels), and using a simple 3-phase SFH (right panels); see text.

Top: Model spectra of the scenarios after 15 Gyr (black) against observed spectrum (grey). Bottom: Photometric evolution of the scenarios in terms of $(\mathrm{B}-\mathrm{V})_{H S T},(\mathrm{~V}-\mathrm{R})_{H S T}$, and $(\mathrm{B}-\mathrm{R})_{H S T}$ with the corresponding SFHs; the observed colours (obtained from the observed spectrum) are marked with black dots at 15 Gyr. 

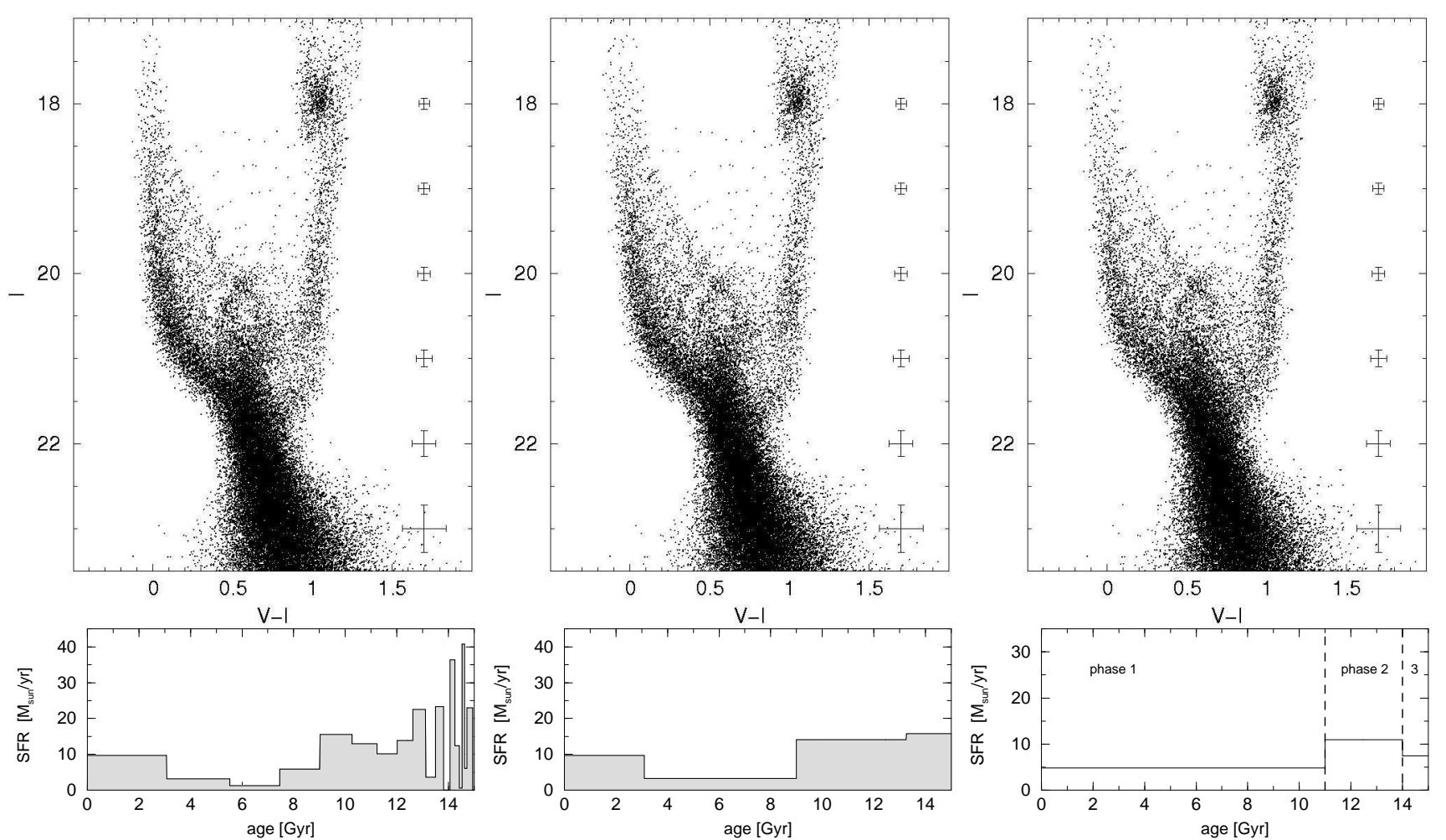

Fig. 2.17: Confrontation of scenarios using Smecker-Hane et al.'s (2002) original SFH (left panels), a simplified Smecker-Hane SFH (central panels), and using a simple 3-phase SFH (right panels); see text.

Top: Model CMDs at a simulated galaxy age of 15 Gyr. Bottom: Corresponding SFHs. 
The longer lookback times in all cases refer to a long wavelength basis and particularly ideal SF scenarios: E.g., all estimates presented in this paper are at fixed metallicity. In the presence of metallicity differences between two scenarios or metallicity evolution between the SF episodes within a given scenario, the uncertainty increases and can only partly be overcome with the use of a very long wavelength basis (UV through NIR).

Hence, we conclude that $S F H$ details can be recovered with similar accuracy from broad band colors or SEDs, from Lick indices, and from low/intermediate resolution spectroscopy (cf. also Cardiel et al. 2003).

The observational study comparing SFHs for an LMC bar field derived from a deep HST CMD by Smecker-Hane et al. (2002) and from an integrated-light ESO spectrum $(4000-8000 \AA)$ yielded the following results:

We constructed a toy model having only three different phases of constant SFRs: The first phase over a period of $11 \mathrm{Gyr}$, the second one over the next 3 Gyr and the third one over the last 1 Gyr. Comparing model spectra with the ESO spectrum, we found that only relative amounts of stars formed in the three phases are relevant for the degree of agreement between model and observed spectra. How the SFR is distributed within each phase does not matter at all - whether it is concentrated at the onset or end of a phase or constant over all of the respective phase. Only within phase 3 , i.e. within the latest 1 Gyr of SF the distribution becomes important.

The agreement with the observed spectrum that we reached with an appropriate three phase toy model is comparable to the agreement reached with Smecker-Hane et al.'s more complicated SFH derived from the CMD.

The CMD that we modeled on the basis of Smecker-Hane et al.'s fairly complex SFH agrees very well with a simplified 4-phase model derived from this $\mathrm{SFH}$, but differs from our 3-phase toy model. A quantitative assessment of this comparison still needs to be done.

From all of these studies, we finally conclude: Both from CMDs and integrated light (multi-band photometry as well as spectroscopy)

1. SFRs during the last Gyr are very precisely recovered,

2. SFRs between 1 and 3 Gyr ago are roughly recovered, and

3. SFRs longer than 3 to 5 Gyr ago are only vaguely recovered,

with "roughly" and "vaguely" meaning that only the relative amounts of stars formed in the three different intervals can be determined, but not the details of the distribution of this SF within the respective interval. Within this frame, integrated light is more sensitive to the latest $1 \mathrm{Gyr}$ of SF, CMDs are more sensitive to intermediate ages.

\subsection{References}

Alloin D., Gallart C., Fleurence E., Pompei E., Raimann D., Fritze-v. Alvensleben U. and Yi S., 2002, Ap\&SS 281, 109

Anders P. and Fritze-v. Alvensleben U., 2003, A\&A 401, 1063 
Bertelli G., Bressan A., Chiosi C., Fagotto F., and Nasi E., 1994, A\&AS 106, 275

Bicker J., Fritze-v. Alvensleben U. and Fricke K.J., 2002, A\&A 387, 412

Bicker J., Fritze-v. Alvensleben U., Möller C.S., and Fricke K.J., 2004, A\&A 413,37

Bruzual A.G., 2001, Ap\&SS 277, 221

Bruzual A.G., 2007, astro-ph/0702091

Cardelli J.A., Clayton G.C., and Mathis J.S., 1989, ApJ 345, 245

Cardiel N., Gorgas J., Sanchez-Blazquez P., et al., 2003, A\&A 409, 511

Cerviño M., Luridiana V. and Castander F.J., 2000, A\&A 360, L5

Chabrier G. and Baraffe I., 1997, A\&A 327, 1039

Charlot S., Worthey G. and Bressan A., 1996, ApJ 457, 625

Holtzman J.A., 2005, Resolved Stellar Populations, ASP Conference Series, Vol. TBA, Valls-Gabaud D. \& Chavez M. (eds)

Lançon A., 1999, IAU Symp. 191, 579

Lejeune T., Cuisinier F., and Buser R., 1997, A\&AS 125, 229

Lejeune T., Cuisinier F., and Buser R., 1998, A\&AS 130, 65

Lilly T., 2003, Master's thesis, University Observatory Göttingen (Germany)

Lilly T. and Fritze-v. Alvensleben U., 2005a, astro-ph/0507303

Lilly T. and Fritze-v. Alvensleben U., 2005b, astro-ph/0507305

Lilly T. and Fritze-v. Alvensleben U., 2006, A\&A 457, 467

Salpeter E.E., 1955, ApJ 121, 161

Schlegel D.J., Finkbeiner D.P., and Davis M., 1998, ApJ 500, 525

Schulz J., Fritze-v. Alvensleben U., Möller C.S., and Fricke K.J., 2002, A\&A 392,1

Smecker-Hane T.A., Cole A.A., Gallagher J.S., and Stetson P.B., 2002, ApJ 566,239

Tinsley B.M., 1978, IAUS 77, 15

Tinsley B.M., 1980, Fund. Cosmic Phys. 5, 287

Vazdekis A., Salaris M., Arimoto N. and Rose J.A., 2001, ApJ 549, 274

Worthey G., 1994, ApJS 95, 107

Worthey G., Faber S.M., González J.J. and Burstein D., 1994, ApJS 94, 687

Worthey G. and Ottaviani D.L., 1997, ApJS 111, 377

Yi S., 2002, Ap\&SS 281, 203 



\title{
Chapter 3
}

\section{Analysing globular cluster observations I: Models and analysis tools for Lick/IDS indices $^{1}$}

\begin{abstract}
We have extended our evolutionary synthesis code, GALEV, to include Lick/IDS absorption-line indices for both simple and composite stellar population models (star clusters and galaxies), using polynomial fitting functions. We present a mathematically advanced Lick index analysis tool (LINO) for the determination of the ages and metallicities of globular clusters (CGs). An extensive grid of GALEV models for the evolution of star clusters at various metallicities over a Hubble time is compared to observed sets of Lick indices of varying completeness and precision. A dedicated $\chi^{2}-$ minimisation procedure selects the best model including $1 \sigma$ uncertainties on age and metallicity. We discuss the age and metallicity sensitivities of individual indices and show that these sensitivities themselves depend on age and metallicity; thus, we extend Worthey's (1994) concept of a "metallicity sensitivity parameter" for an old stellar population at solar metallicity to younger clusters of different metallicities. We find that indices at low metallicity are generally more age sensitive than at high metallicity. Our aim is to provide a robust and reliable tool for the interpretation of star-cluster spectra becoming available from $10 \mathrm{~m}$ class telescopes in a large variety of galaxies - metal-rich 83 metal-poor, starburst, post-burst, and dynamically young. We test our analysis tool using observations from various authors for Galactic and M31 GCs, for which reliable age and metallicity determinations are available in the literature, and discuss to what extent the observational availability of various subsets of Lick indices affects the results. For M31 GCs, we discuss the influence of non-solar abundance ratios on our results. All models are accessible from our website, http://www.astro.physik.uni-goettingen.de/ galev/
\end{abstract}

${ }^{1}$ This chapter is published as Lilly and Fritze-v. Alvensleben, 2006, A\&A 457, 467 


\subsection{Introduction}

In order to understand the formation and evolution of galaxies, one of the essential issues is to reveal their star formation histories ( $\mathrm{SFHs}$ ). Unfortunately, most galaxies are observable only in integrated light, so that SFH determinations using the most reliable CMD approach are only possible for a very limited sample of nearby galaxies. However, the age and metallicity distributions of star cluster and globular cluster (GC) systems can provide important clues to the formation and evolutionary history of their parent galaxies. For example, the violent formation history of elliptical galaxies, as predicted from hierarchical or merger scenarios, is, in fact, obtained more directly from the age and metallicity distributions of their GC systems than from their integrated spectra, which are always dominated by stars originating in the last major star-formation episode. By means of evolutionary synthesis models, for example, we can show that, when using the integrated light of a galaxy's (composite) stellar content alone, it is impossible to date (and actually to identify) even a very strong starburst if this event took place more than two or three Gyrs ago (Lilly 2003, Lilly \& Fritze - von Alvensleben 2005). Therefore, it is an important first step towards understanding the formation and evolution of galaxies to constrain the age and metallicity distributions of their star cluster systems (for recent reviews see, for example, Kissler-Patig 2000 and Zepf 1999, 2002), as well as of their stars (see, e.g., Harris et al. 1999, Harris \& Harris 2000, 2002). Star clusters can be observed one-by-one to fairly high precision in galaxies out to Virgo cluster distances, even on bright and variable galaxy backgrounds, in terms of both multi-band imaging and intermediate-resolution spectroscopy. For young star cluster systems, we have shown that the age and metallicity distributions can be obtained from a comparison of multi-band imaging data with a grid of model SEDs using the SED analysis tool AnalySED (Anders et al. 2004).

Our aim is to extend the analysis of star cluster age and metallicity distributions in terms of parent galaxy formation histories and scenarios to intermediate age and old star cluster systems. However, for all colours the evolution slows down considerably at ages older than about 8 Gyr. Even with several passbands and a long wavelength basis, the results are extremely uncertain for old GCs; colours - even when combining optical and near infrared - do not allow the agemetallicity degeneracy to be completely disentangled (cf. Anders et al. 2004). Absorption-line indices, on the other hand, are a promising tool for independent and more precise constraints on ages and metallicities. Therefore, we present a grid of new evolutionary synthesis models for star clusters, including Lick/IDS indices, to complement the broad band colours and spectra of our previous models, and a Lick index analysis tool LINO meant to complement our SED analysis tool. With these two analysis tools, we now possess reasonable procedures for interpreting both broad-band colour and spectral index observations.

In an earlier study, we already incorporated a subset of Lick indices into our evolutionary synthesis code GALEV (Kurth et al. 1999). However, since then the input physics for the code has changed considerably; instead of the older tracks, we are now using up-to-date Padova isochrones, which include the thermally pulsing asymptotic giant branch (TP-AGB) phase of stellar evolution (see Schulz et al. 2002). In this work, we present the integration of the full set of Lick indices into our code. Now, our GALEV models consistently describe the time evolution of spectra, broad-band colours, emission lines, and Lick indices 
for both globular clusters (treated as single-age single-metallicity, i.e. "simple" stellar populations, SSPs) and galaxies (composite stellar populations, CSPs), using the same input physics for all models (for an exhaustive description of GALEV and its possibilities, as well as for recent extensions of the code and its input physics, see Schulz et al. 2002, Anders \& Fritze - v. Alvensleben 2003, and Bicker et al. 2004).

A recent publication (Proctor et al. 2004) also presented an analysis tool for Lick indices using an $\chi^{2}$-approach. However, they do not provide any confidence intervals for their best-fitting models. In this respect, our new tool extends their approach. A drawback of our models is that, at the present stage, they do not account for variations in $\alpha$-enhancement, as Proctor et al. (2004) do. However, our analysis tool $L I N O$ is easily applicable to any available set of absortion line indices.

In Sect. 3.2, we recall the basic definitions of Lick indices and describe how we synthesize them in our models; we also address non-solar abundance ratios. Some examples of SSP model indices are presented and briefly confronted with observations. In Sect. 3.3, Worthey's (1994) "metallicity sensitivity parameter" is discussed and extended from old stellar populations to stellar populations of all ages. Section 3.4 describes and tests our new Lick index analysis tool; Galactic and M31 globular cluster observations are analysed and compared with results (taken from the literature) from reliable CMD analysis, and from index analyses using models with varying $\alpha$-enhancements, respectively. Section 3.5 summarises the results and provides an outlook.

\subsection{Models and input physics}

In this section, we give an overview of our GALEV models and describe how we synthesize Lick indices. We address the impact of non-solar abundance ratios on our results and compare some examples of SSP model indices with observations.

\subsubsection{Evolutionary synthesis of Lick indices}

Lick indices are relatively broad spectral features, and robust to measure. They are named after the most prominent absorption line in the respective index's passband. However, this does not necessarily mean that a certain index's strength is exclusively, or even dominantly, due to line(s) of this element (see, e.g., Tripicco \& Bell 1995). Beyond the fact that more than one line can be present in the index's passband, strong lines in the pseudo-continua can also affect the index strength. Most indices are given in units of their equivalent width (EW) measured in $\AA$,

$$
\mathrm{EW}[\AA]=\int_{\lambda_{1}}^{\lambda_{2}} \frac{F_{C}(\lambda)-F_{I}(\lambda)}{F_{C}(\lambda)} \mathrm{d} \lambda,
$$

whereas index strengths of broad molecular lines are given in magnitudes:

$$
\mathrm{I}[\mathrm{mag}]=-2.5 \log \left[\left(\frac{1}{\lambda_{1}-\lambda_{2}}\right) \int_{\lambda_{1}}^{\lambda_{2}} \frac{F_{I}(\lambda)}{F_{C}(\lambda)} \mathrm{d} \lambda\right] .
$$


Here, $F_{I}(\lambda)$ is the flux in the index covering the wavelength range between $\lambda_{1}$ and $\lambda_{2}$, and $F_{C}(\lambda)$ is the continuum flux defined by two "pseudo-continua" flanking the central index passband.

There are currently 25 Lick indices, all within the optical wavelength range: $\mathrm{H} \delta_{A}, \mathrm{H} \gamma_{A}, \mathrm{H} \delta_{F}, \mathrm{H} \gamma_{F}, \mathrm{CN}_{1}, \mathrm{CN}_{2}, \mathrm{Ca} 4227, \mathrm{G} 4300, \mathrm{Fe} 4383, \mathrm{Ca} 4455, \mathrm{Fe} 4531$, $\mathrm{Fe} 4668, \mathrm{H} \beta, \mathrm{Fe} 5015, \mathrm{Mg}_{1}, \mathrm{Mg}_{2}, \mathrm{Mg} b, \mathrm{Fe} 5270, \mathrm{Fe} 5335, \mathrm{Fe} 4506, \mathrm{Fe} 5709, \mathrm{Fe} 5782$, $\mathrm{Na} \mathrm{D}, \mathrm{TiO}_{1}$, and $\mathrm{TiO}_{2}$. For a full description and all index definitions, see Trager et al. (1998) and references therein.

As the basis for our evolutionary synthesis models, we employ the polynomial fitting functions of Worthey et al. (1994) and Worthey \& Ottaviani (1997), which give Lick index strengths of individual stars as a function of their effective temperature $T_{\text {eff }}$, surface gravity $g$, and metallicity $[\mathrm{Fe} / \mathrm{H}]$. Worthey et al. have calibrated their fitting functions empirically using solar-neighbourhood stars.

Model uncertainties are calculated as follows (Worthey 2004):

$$
\sigma_{\text {model }}=\frac{\sigma_{\text {star }} \times \mathrm{RMS}_{\mathrm{fit}}}{\sqrt{N}}
$$

with $\sigma_{\text {star }}$ the typical rms error per observation for the calibration stars and $\mathrm{RMS}_{\text {fit }}$ the residual rms of the fitting functions; both values are given in Worthey et al. (1994) and Worthey \& Ottaviani (1997). Here, $\mathrm{N}$ is the number of stars in the "neighbourhood" of the fitting functions in the $T_{\text {eff }}, g,[\mathrm{Fe} / \mathrm{H}]$ space, which is typically of the order of 25 . Note that this approach is only an approximation; the real model error is most likely a strong function of $T_{\text {eff }}, g$, and $[\mathrm{Fe} / \mathrm{H}]$.

Other input physics of our models include the theoretical spectral library from Lejeune et al. $(1997,1998)$, as well as theoretical isochrones from the Padova group for $Z=0.0004,0.004,0.008,0.02$, and 0.05 (cf. Bertelli et al. 1994), and for $Z=0.0001$ (cf. Girardi et al. 1996). Recent versions of these isochrones include the TP-AGB phase of stellar evolution (not presented in the referenced papers), which is important for intermediate age stellar populations (cf. Schulz et al. 2002). We assume a standard Salpeter (1955) initial mass function (IMF) from 0.15 to about $70 \mathrm{M}_{\odot}$; lowest mass stars $\left(\mathrm{M}_{\odot}<0.6\right)$ are taken from Chabrier and Baraffe (1997) (cf. Schulz et al. 2002 for details). Throughout this paper, we identify the metallicity $Z$ with $[\mathrm{Fe} / \mathrm{H}]$ and define $[\mathrm{Fe} / \mathrm{H}]:=\log \left(Z / Z_{\odot}\right)$.

To calculate the time evolution of Lick indices for SSP or galaxy models, we follow four steps:

1. We use the values for $T_{\text {eff }}, g$, and $[\mathrm{Fe} / \mathrm{H}]$ given (directly or indirectly) by the isochrones to compute the index strength $\mathrm{EW}_{\text {star }}$ or $\mathrm{I}_{\text {star }}$ for each star along the isochrones.

2. A spectrum is assigned to each star on a given isochrone and used to compute its continuum flux $F_{C}{ }^{2}$.

3. For each isochrone, the index strengths are integrated over all stellar masses $m$ (after transformation of the index strengths into fluxes), weighted

\footnotetext{
${ }^{2}$ In view of the resolution of our spectral library, these values are not very accurate; however, since $F_{C}$ is merely an additional weighting factor for the integration routine, this does not affect the final results.
} 
by the IMF (using a weighting factor $w$ )

$$
\mathrm{EW}_{\mathrm{SSP}}=\left(\lambda_{2}-\lambda_{1}\right) \cdot\left(1-\frac{\sum_{m}\left(F_{I} \cdot w\right)}{\sum_{m}\left(F_{C} \cdot w\right)}\right),
$$

where $F_{I}$ is a function of $\mathrm{EW}_{\text {star }}$ and $F_{C}$ :

$$
F_{I}=F_{C} \cdot\left(1-\frac{\mathrm{EW}_{\mathrm{star}}}{\lambda_{2}-\lambda_{1}}\right) .
$$

The result of these 3 steps is a grid of SSP models for all available isochrones, i.e., a grid consisting of the 6 metallicities given above and 50 ages between 4 Myr and 20 Gyr.

4. For each time step in the computation of a stellar population model, our evolutionary synthesis code GALEV gives the contribution of each isochrone to the total population.

To obtain galaxy model indices (or a better age resolution for SSP models), we integrate our grid of SSP models using Eqs. (3.4) and (3.5) again, but with $w$ the isochrone contribution as a new weighting factor (now doing the summation over all isochrones instead of all masses), $F_{C}$ the integrated continuum flux level for each isochrone, and using $\mathrm{EW}_{\mathrm{SSP}}$ instead of $\mathrm{EW}_{\text {star }}$.

This way, we computed a large grid of SSP models, consisting of 6 metallicities and 4000 ages from 4 Myr to 16 Gyr in steps of 4 Myr; each point of the model grid consists of all the 25 Lick indices currently available.

\subsubsection{Non-solar abundance ratios}

Abundance ratios reflect the relation between the characteristic time scale of star formation and the time scales for the release of, e.g., SNe II products (Mg and other $\alpha$-elements), SNe Ia products (Fe), or nucleosynthetic products from intermediate-mass stars $(\mathrm{N})$. Galaxies with different SFHs will hence be characterised by different distributions of stellar abundances ratios. This means that the Galactic relation between abundance ratios and metallicity (Edvardsson et al. 1993, Pagel \& Tautvaišiene 1995) is not necessarily valid for galaxies of different types and formation histories. Empirical index calibrations based on Galactic stars, like the fitting functions from Worthey et al. (1994) and Worthey \& Ottaviani (1997) applied in this work, are based on the implicit inclusion of the Galactic relation between abundance ratios and metallicity.

A lot of work has been done in past years to study the impact of $\alpha$-enhancement on stellar population models and their applications; e.g., based mainly on the work of Tripicco \& Bell (1995) and Trager et al. (2000a), Thomas et al. (2003, 2004 ) present SSP models of Lick indices with variable abundance ratios that are corrected for the bias mentioned above, providing for the first time well-defined $[\alpha / \mathrm{Fe}]$ ratios at all metallicities. The impact of these new models on age and metallicity estimates of early type galaxies is investigated in detail by Maraston et al. (2003), Thomas \& Maraston (2003), Thomas et al. (2004), Trager et al. (2000a,b), among others. 
However, since our purpose is to present consistently computed models for spectra, colours, emission lines, and Lick indices for both SSPs and CSPs, a consistent attempt to allow our evolutionary synthesis code GALEV to account for arbitrary abundance ratios would have to be based on stellar evolutionary tracks or isochrones, detailed nucleosynthetic stellar yields, and model atmospheres for various abundance ratios. Since both consistent and complete datasets of this kind are not yet available (though first sets of evolutionary tracks for stars with enhanced $[\alpha / \mathrm{Fe}]$ ratios were presented by Salasnich et al. 2000 and Kim et al. 2002 ), our models at the present stage do not explicitly allow for variations in $\alpha$-enhancement. This is an important caveat to be kept in mind for the interpretation of extragalactic GC populations. We think that the extensive studies of non-solar abundance ratios cited above will allow us to estimate the impact of this caveat on our results.

However, in Sect. 3.4.3 we show that our method is robust enough to give very good age and metallicity determinations for GCs even without using $\alpha$ enhanced models.

\subsubsection{SSP model indices: Some examples}

In Figs. 3.1 and 3.2, we show the time evolution and metallicity dependence of the indices $\mathrm{H} \beta$ and Fe5335 in our new SSP models, and compare them with index measurements of Galactic GCs that are plotted against reliable age and metallicity estimates, respectively.

In particular, in Fig. 3.1 we compare SSP models for five ages between 1 and 16 Gyr with Galactic GC observations by Burstein et al. (1984; 17 clusters), Covino et al. (1995; 17 clusters), Trager et al. (1998; 18 clusters), Puzia et al. (2002; 12 clusters), and Beasley et al. (2004; 12 clusters). Note that some clusters were observed repeatedly, so more than one data point in the figure can refer to the same cluster. The metallicities are taken from Harris (1996, revision Feb. 2003). In Fig. 3.2 we show the time evolution of the model indices for all six metallicities, and compare them with Galactic GC observations (taken from the same references as in Fig. 3.1). The GC age determinations are based on CMD fits and taken from Salaris \& Weiss $(2002)^{3}$. Over the range of Galactic GC ages and metallicities (i.e., ages older than about $8 \mathrm{Gyr}$ and metallicities lower than solar in most cases), a sufficient agreement is observed between models and data in the sense that the data lie within the range of the model grid; we also checked this for other indices (not plotted).

However, the plots also demonstrate how difficult it would be to interpret the indices in terms of classical index-index plots. Actually, Fig. 3.2 seems to show apparent inconsistencies, so some clusters in Fig. 3.2 have metallicities up to $[\mathrm{Fe} / \mathrm{H}]=+0.4$ when compared with models for the age-sensitive index $\mathrm{H} \beta$, whereas, when compared with models for the metallicity-sensitive Fe5335 index, all clusters have metallicities lower than $[\mathrm{Fe} / \mathrm{H}]=-0.4$.

\footnotetext{
${ }^{3}$ Note that they only cover a subsample of the observations shown in Fig.3.1: 11 clusters of the Burstein et al. (1984) sample, 10 of the Covino et al. (1995) sample, 10 of the Trager et al. (1998) sample, only 3 of the Puzia et al. (2002) sample, and 6 clusters of the Beasley et al. (2004) sample.
} 

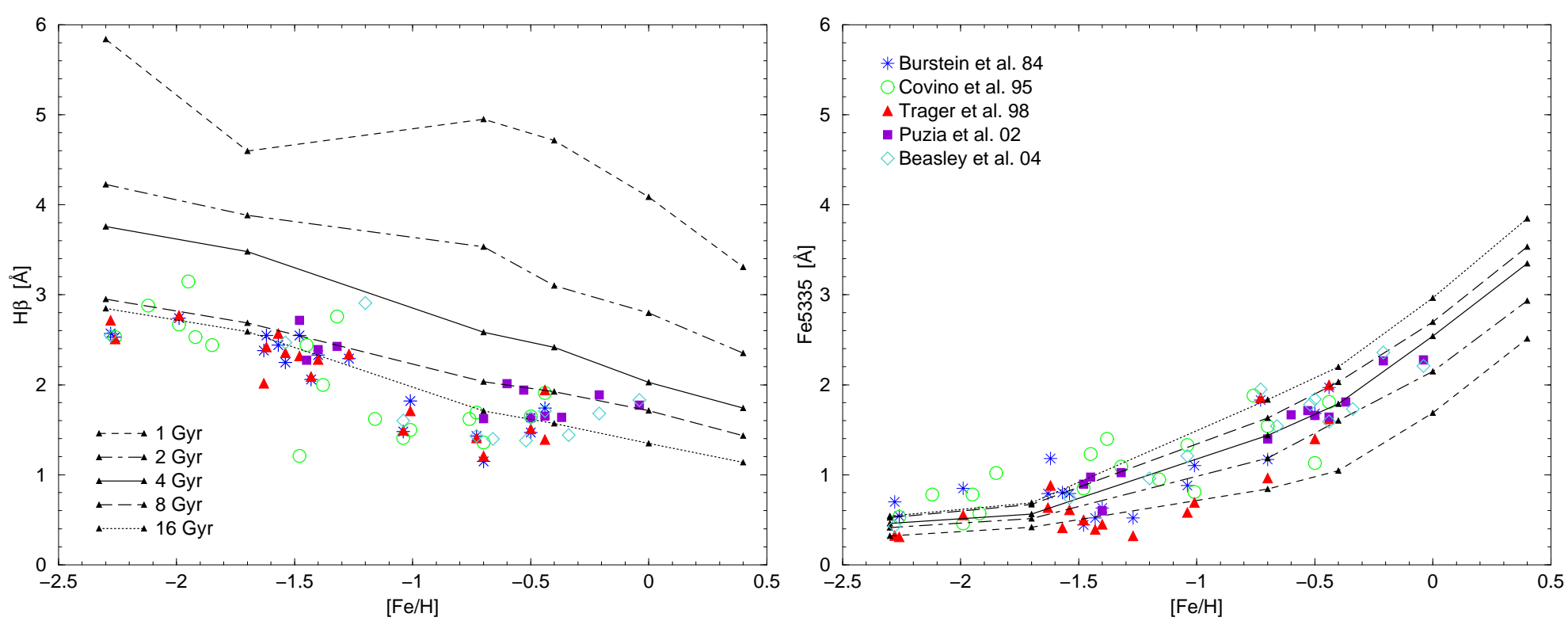

Fig. 3.1: Indices $\mathrm{H} \beta$ (left) and Fe5335 (right) versus metallicity for 5 different ages. Also shown are Galactic GC observations from various authors as indicated in the right-hand panel; GC metallicities are taken from Harris (1996, revision Feb. 2003). A typical measurement error is about $0.2 \AA$. 

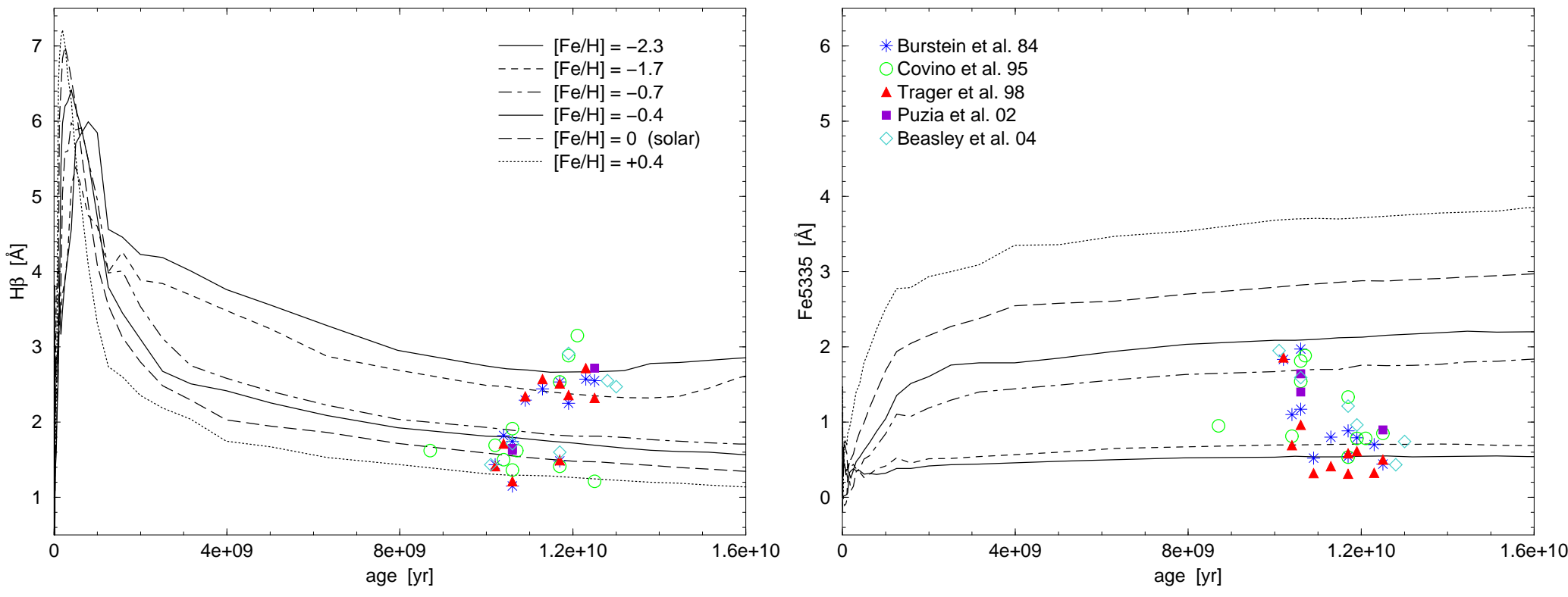

Fig. 3.2: Indices $\mathrm{H} \beta$ (left) and Fe5335 (right) versus age for 6 different metallicities. Also shown are Galactic GC observations from various authors as indicated in the right-hand panel; GC age determinations are taken from Salaris \& Weiss (2002). 
We cannot decide at this point to what degree these inconsistencies are due to problems in the models or the calibrations they are based on or due to badly calibrated observations; however, our new Lick index analysis tool nevertheless gives surprisingly robust age and, particularly, metallicity determinations for the same set of cluster observations (cf. Sect. 3.4.2).

\subsection{Index sensitivities}

It is well known that different indices have varying sensitivities to age and/or metallicity. To quantify this, Worthey (1994) introduced a "metallicity sensitivity parameter" that gives a hint of how sensitive a given index is with respect to changes in age and metallicity. This parameter is defined as the ratio of the percentage change in $\mathrm{Z}$ to the percentage change in age (so influences of possible age-metallicity degeneracies are implicitely included), with large numbers indicating greater metallicity sensitivity:

$$
S=\left(\frac{\Delta I_{Z}}{\Delta Z / Z}\right) /\left(\frac{\Delta I_{\text {age }}}{\Delta a g e / a g e}\right) .
$$

Using his SSP models, Worthey (1994) chose a 12 Gyr solar metallicity $(\mathrm{Z}=0.017)$ model as the zero point for the sensitivity parameters, the $\Delta$ 's referring to "neighbouring" models, in this case models with age $=8 / 17$ Gyr (i.e, $\Delta$ age $=4 / 5$ Gyr) and $\mathrm{Z}=0.01 / 0.03$ (i.e., $\Delta \mathrm{Z}=0.007 / 0.013)^{4}$; the main numerator/denominator in Eq. 3.6 is averaged using both $\Delta$ 's before computing the fraction.

In Table 3.1, we reprint the metallicity sensitivity parameters given by Worthey (1994) and Worthey \& Ottaviani (1997), and compare them with parameters computed using our own models. Extending Worthey's approach, we computed parameters for four different combinations of zero points, using high $(\mathrm{Z}=0.02)$ and low $(\mathrm{Z}=0.0004)$ metallicities, along with high $(12 \mathrm{Gyr})$ and intermediate (4 Gyr) ages.

Worthey's parameters are reproduced relatively well by models with a similar combination of zero points, i.e. for old (12 Gyr) and solar metallicity SSPs. However, $\mathrm{S}$ is not totally independent of the $\Delta \mathrm{Z}$ and $\Delta$ age chosen, since it can be very sensitive to the exact evolution of the model index. This occurs mainly in age-metallicity space regions where the slope of the index does not evolve very smoothly. For example, a very high value of $\mathrm{S}$ can also mean that, due to a small "bump" in the time evolution of the index, $\Delta I_{a g e}$ is near zero; in this case, $\mathrm{S}$ is worthless.

The two zero points for both age and metallicity and their "neighbouring models" we use for the computations are given in Table 3.2; for both age zero points, we chose two sets of neighbouring models and averaged the final parameters. To check the reliability of our results, we also computed parameters for values of age and $\Delta$ age not given in the table. If the results for different $\Delta$ age's (or slightly different zero points) differ strongly, we classify the parameter as uncertain (indicated by brackets in Table 3.1). The "ranking" of indices in terms of sensitivity is, with some exceptions, unaffected by changes in the zero points.

\footnotetext{
${ }^{4}$ Ideally, $\mathrm{S}$ should be relatively independent of the exact values of the $\Delta \mathrm{Z}$ and $\Delta$ age chosen, as long as they are not too large.
} 
Table 3.1: Metallicity sensitivity parameters for different zero points. Low numbers indicate high age sensitivity. Values given in brackets are not reliable (see text).

\begin{tabular}{cccccc}
\hline \hline & WORTHEY & \multicolumn{2}{c}{ GALEV 12 Gyr } & \multicolumn{2}{c}{ GALEV 4 Gyr } \\
& & $\mathrm{Z}=0.02$ & 0.0004 & 0.02 & 0.0004 \\
\hline $\mathrm{CN}_{1}$ & 1.9 & 1.5 & 1.1 & 1.1 & 0.1 \\
$\mathrm{CN}_{2}$ & 2.1 & 1.5 & 0.2 & 1.2 & 0.1 \\
$\mathrm{Ca} 4227$ & 1.5 & 1.1 & $(0.4)$ & 1.0 & 0.1 \\
$\mathrm{G} 4300$ & 1.0 & 1.0 & 0.2 & 0.8 & 0.1 \\
$\mathrm{Fe} 4383$ & 1.9 & 1.9 & 0.3 & 1.3 & 0.2 \\
$\mathrm{Ca} 4455$ & 2.0 & 1.7 & 0.7 & 1.5 & 0.3 \\
$\mathrm{Fe} 4531$ & 1.9 & 1.7 & 0.6 & 1.4 & 0.2 \\
$\mathrm{Fe} 4668$ & 4.9 & $(3.5)$ & $(0.9)$ & 2.4 & 0.9 \\
$\mathrm{H} \beta$ & 0.6 & 0.6 & 0.1 & 0.5 & 0.1 \\
$\mathrm{Fe} 5015$ & 4.0 & $(2.3)$ & $(1.3)$ & 2.1 & 0.4 \\
$\mathrm{Mg}$ & 1.8 & 1.7 & $(2.2)$ & 1.4 & 2.0 \\
$\mathrm{Mg} g_{2}$ & 1.8 & 1.5 & $(1.8)$ & 1.2 & 0.5 \\
$\mathrm{Mg} b$ & 1.7 & 1.4 & $(0.7)$ & 1.0 & 0.3 \\
$\mathrm{Fe} 5270$ & 2.3 & 2.0 & $(0.7)$ & 1.6 & 0.3 \\
$\mathrm{Fe} 5335$ & 2.8 & 2.7 & $(1.3)$ & 2.0 & 0.4 \\
$\mathrm{Fe} 5406$ & 2.5 & $(2.6)$ & $(2.3)$ & 1.8 & 0.6 \\
$\mathrm{Fe} 5709$ & 6.5 & $(8.5)$ & $(1.7)$ & 2.6 & $(1.2)$ \\
$\mathrm{Fe} 5782$ & 5.1 & $(5.9)$ & $(1.4)$ & 2.5 & $(1.0)$ \\
$\mathrm{Na} \mathrm{D}$ & 2.1 & 1.9 & $(1.2)$ & 1.9 & 0.6 \\
$\mathrm{TiO} \mathrm{O}_{1}$ & 1.5 & 0.9 & 0.7 & $(1.4)$ & $(5.5)$ \\
$\mathrm{TiO}{ }_{2}$ & 2.5 & 1.3 & 0.9 & $(1.6)$ & $(8.6)$ \\
$\mathrm{H} \delta_{A}$ & 1.1 & 1.0 & 0.3 & 0.8 & 0.1 \\
$\mathrm{H} \gamma_{A}$ & 1.0 & 1.0 & 0.2 & 0.8 & 0.1 \\
$\mathrm{H} \delta_{F}$ & 0.9 & 0.9 & 0.1 & 0.7 & 0.1 \\
$\mathrm{H} \gamma_{F}$ & 0.8 & 0.8 & 0.2 & 0.7 & 0.1 \\
\hline
\end{tabular}

Contrary to our expectations, however, at solar metallicity the age-sensitivity of Lick indices is only slightly higher for an intermediate age model compared to the 12 Gyr model; for low-metallicity SSPs, the effect is more pronounced. Most important, we find that for models at low metallicity, indices are generally much more age-sensitive than for models at high metallicity, especially for agesensitive indices like G4300 or Balmer line indices. This means that the indices of old, low metallicity GCs can be more sensitive to age than indices of GCs with high metallicity and intermediate age. This is of special interest for any analysis of GC systems involving intermediate age GCs (e.g., in merger remnants), since secondary GC populations with intermediate ages are generally expected to have higher metallicities than "normal" old and metal-poor populations.

Given the limited accuracy of any index measurement in practice, the usefulness of an index to determine age or metallicity depends not only on the relative change in index strength for changing $\mathrm{Z}$ or age as it is given by $\mathrm{S}$ but also on the absolute change in index strength.

Therefore, in Fig. 3.3 we show the absolute differences of index strengths for 
Table 3.2: "Neighbouring models" in terms of metallicity for $\mathrm{Z}=0.02$ and $\mathrm{Z}=0.0004$ (top) and in terms of age for the zero points 12 Gyr and 4 Gyr (bottom) used to compute the metallicity sensitivities given in Table 3.1. Brackets give the corresponding $\Delta \mathrm{Z}$ and $\Delta$ age.

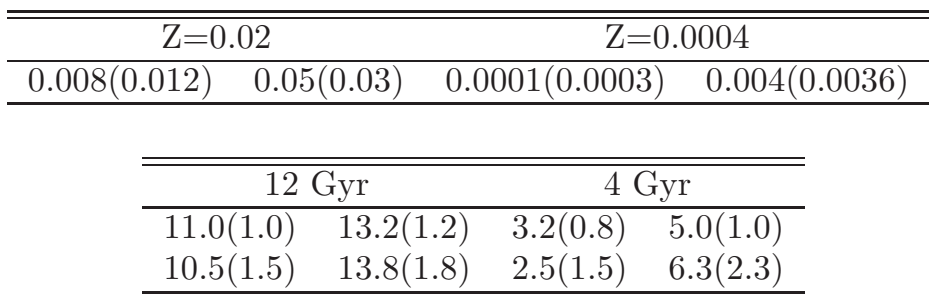

old (12 Gyr) and young (2 Gyr) SSPs for changing metallicity and for metal-rich $([\mathrm{Fe} / \mathrm{H}]=0)$ and metal-poor $([\mathrm{Fe} / \mathrm{H}]=-1.7)$ SSPs for changing age, respectively. Generally, the absolute differences between 8 and 4 Gyr old SSPs are larger than the differences between 8 and 12 Gyr old SSPs at fixed metallicity, as expected (Fig. 3.3, lower panels). However, this effect is much stronger at low than at high metallicity, which confirms what we get from the S parameter. The absolute differences between models with different metallicity (top panels in Fig. 3.3) are slighty larger for old than for young SSPs. Interestingly, the plots show that indices known to be sensitive to age can also be highly variable for differing metallicities; especially the broad Balmer indices $\mathrm{H} \delta_{A}$ and $H \gamma_{A}$ change a lot with metallicity. Most important, however, the plot shows that, in practice, moderately metal-sensitive indices like Mgb can be much more useful for metallicity determinations than indices like Fe5709 or Fe5782, though the latter are, according to the $\mathrm{S}$ parameter, much more metal-sensitive.

In order to determine ages and metallicities of GCs, indices should be chosen not only according to known sensitivities as given by $\mathrm{S}$, but also according the achievable measurement accuracy and, if possible, according to the expected age and metallicity range of the sources. 

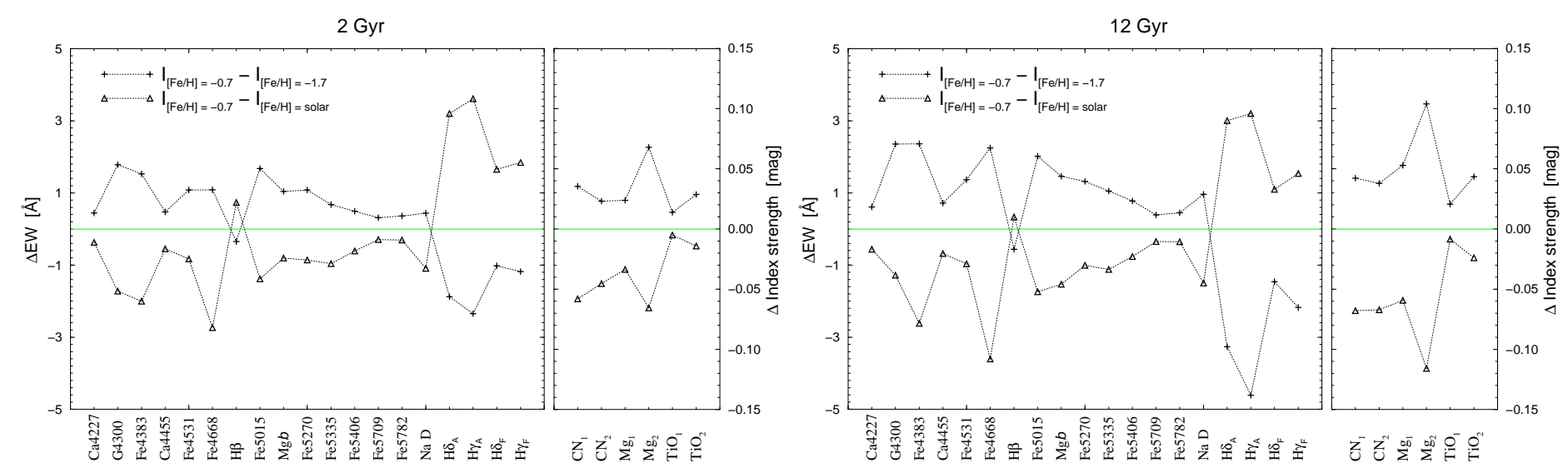

Fig. 3.3: Absolute differences of index strengths for old and young SSP models for changing metallicity (top), and for metal-rich and metal-poor SSP models for changing age (bottom). The dotted lines are just for presentation. 


\subsection{The Lick index analysis tool}

Since in the original models (cf. Sect. 3.2.1), the steps in metallicity are large, in a first step we linearly interpolate in $[\mathrm{Fe} / \mathrm{H}]$ between the 6 metallicities before we analyse any data with our new tool. This is done in steps of $[\mathrm{Fe} / \mathrm{H}]=0.1$ dex, so the final input grid for the analysis algorithm consists of sets of all 25 Lick indices each for 28 metallicities $(-2.3 \leq[\mathrm{Fe} / \mathrm{H}] \leq+0.4)$ and 4000 ages $(4$ $\mathrm{Myr} \leq$ age $\leq 16 \mathrm{Gyr}$ ). Although this approach is only an approximation, the results shown in Sect. 3.4.2 prove it to be sufficiently accurate.

\subsubsection{The $\chi^{2}$ - approach}

The algorithm is based on the SED analysis tool presented by Anders et al. (2004). The reader is referred to this paper for additional information about the algorithm, as well as for extensive tests using broad-band colours instead of indices.

All observed cluster indices at once - or an arbitrary subsample of them are compared with the models by assigning a probability $p(n)$ to each model grid point (i.e., to each set of 25 indices defined by 1 age and 1 metallicity)

$$
p(n) \propto\left(-\chi^{2}\right)
$$

where

$$
\chi^{2}=\sum_{i=1}^{25} \frac{\left(I_{\mathrm{obs}}-I_{\text {model }}\right)^{2}}{\sigma_{\mathrm{obs}}^{2}+\sigma_{\text {model }}^{2}}
$$

with $I_{\mathrm{obs}}$ and $I_{\text {model }}$ the observed and the model indices, respectively, and $\sigma_{\text {obs }}$ and $\sigma_{\text {model }}$ the respective uncertainties. Indices measured in magnitudes are transformed into Ångström before calculation. After normalization $\left(\sum p(n)=\right.$ 1 ), the grid point with the highest probability is assumed to be the best model, i.e. it gives the "best age" and the "best metallicity" for the observed cluster.

The uncertainties of the best model in terms of $\pm 1 \sigma$ confidence intervals are computed by rearranging the model grid points by order of decreasing probabilities, and summing up their probabilities until $\sum p(n)=0.68$ is reached; the $1 \sigma$ uncertainties in age and metallicity are computed from the age and metallicity differences, respectively, of the $n\left(p_{0.68}\right)$ - and the $n\left(p_{\max }\right)$-model. Note that the determination does not take into account the possible existence of several solution "islands" for one cluster; thus the confidence intervals are in fact upper limits.

\subsubsection{Examples and tests I: Galactic GCs}

We have tested our Lick index analysis tool using a large set of Galactic GCs for which index measurements (taken from Burstein et al. 1984, Covino et al. 1995, Trager et al. $1998^{5}$, and Beasley et al. 2004), as well as age and metallicity determinations from CMD analyses (taken from Salaris \& Weiss 2002) are

\footnotetext{
${ }^{5}$ In this dataset, $\mathrm{H} \delta_{A}, \mathrm{H} \gamma_{A}, \mathrm{H} \delta_{F}$, and $\mathrm{H} \gamma_{F}$ are taken from Kuntschner et al. (2002) who reanalysed the Trager et al. spectra; in the following, 'Trager et al. (1998)' always is meant to include this additional data.
} 
Table 3.3: Observations by Burstein et al. (1984, B84), Covinio et al. (1995, C95), Trager et al. (1998, T98; $\mathrm{H} \delta, \mathrm{H} \gamma$ are taken from Kuntschner et al. 2002, see text), Beasley et al. (2004, B04) used to perform the tests of Sect. 3.4.2. '*': index observed, 'o': only a subsample of clusters is observed in this index.

\begin{tabular}{|c|c|c|c|c|}
\hline & $\overline{B 84}$ & C95 & T98 & B04 \\
\hline $\mathrm{CN}_{1}$ & $*$ & $\mathrm{O}$ & * & O \\
\hline $\mathrm{CN}_{2}$ & & & $*$ & o \\
\hline $\mathrm{Ca} 4227$ & & & $*$ & $\mathrm{O}$ \\
\hline G4300 & $*$ & o & $*$ & o \\
\hline Fe4383 & & & $\mathrm{O}$ & O \\
\hline $\mathrm{Ca} 4455$ & & & $*$ & $\mathrm{O}$ \\
\hline Fe4531 & & & $*$ & o \\
\hline Fe4668 & & & $*$ & $*$ \\
\hline $\mathrm{H} \beta$ & $*$ & $*$ & $*$ & $*$ \\
\hline Fe5015 & & & $*$ & $*$ \\
\hline $\mathrm{Mg}_{1}$ & $*$ & $*$ & $*$ & $*$ \\
\hline $\mathrm{Mg}_{2}$ & $*$ & $*$ & $*$ & $*$ \\
\hline $\mathrm{Mg} b$ & $*$ & $*$ & $*$ & $*$ \\
\hline Fe5270 & $*$ & $*$ & $*$ & $*$ \\
\hline Fe5335 & $*$ & $*$ & $*$ & $*$ \\
\hline Fe5406 & & & $*$ & $*$ \\
\hline Fe5709 & & & $*$ & $*$ \\
\hline Fe5782 & & & $\mathrm{O}$ & $*$ \\
\hline $\mathrm{Na} \mathrm{D}$ & $*$ & $\mathrm{O}$ & $*$ & $*$ \\
\hline $\mathrm{TiO}_{1}$ & $*$ & & $*$ & $*$ \\
\hline $\mathrm{TiO}_{2}$ & o & & O & \\
\hline $\mathrm{H} \delta_{A}$ & & & $*$ & O \\
\hline $\mathrm{H} \gamma_{A}$ & & & o & o \\
\hline $\mathrm{H} \delta_{F}$ & & & $*$ & o \\
\hline $\mathrm{H} \gamma_{F}$ & & & $*$ & $\mathrm{O}$ \\
\hline
\end{tabular}

available.

Figure 3.4 compares ages and metallicities from both methods. Here, we use the complete set of measured indices available (cf. Table 3.3) as input for our analysis tool; for comparison, Fig. 3.5 shows our results using two subsets of indices: the age-sensitive indices $\mathrm{Ca} 4227, \mathrm{G} 4300, \mathrm{H} \beta$, and $\mathrm{TiO}_{1}$ in the left panel, and metal-sensitive indices $\mathrm{Mg}_{1}, \mathrm{NaD},[\mathrm{MgFe}]$, plus the age-sensitive index $\mathrm{H} \beta$ in the right panel ${ }^{6}$. In all plots, only results with confidence intervals of $\sigma$ (age) $\leq 5$ Gyr are plotted ${ }^{7}$.

${ }^{6}[\mathrm{MgFe}]$ is a combination of metal-sensitive indices that is known to be widely unaffected by non-solar abundance ratios (see, e.g., Thomas et al. 2003). It is defined as $[\mathrm{MgFe}]:=$ $\sqrt{<\mathrm{Fe}>\times \mathrm{Mg} b}$, with $<\mathrm{Fe}\rangle:=(\mathrm{Fe} 5270+\mathrm{Fe} 5335) / 2$.

${ }^{7}$ In most cases, very large $1 \sigma$ uncertainties are due to the presence of two "solution islands" (e.g., solution 1: low or intermediate age, solution 2: high age), which are both within their $1 \sigma$ ranges. Since we do not want to use any a priori information about the clusters, we cannot decide between the two solutions and therefore rather omit them completely. 

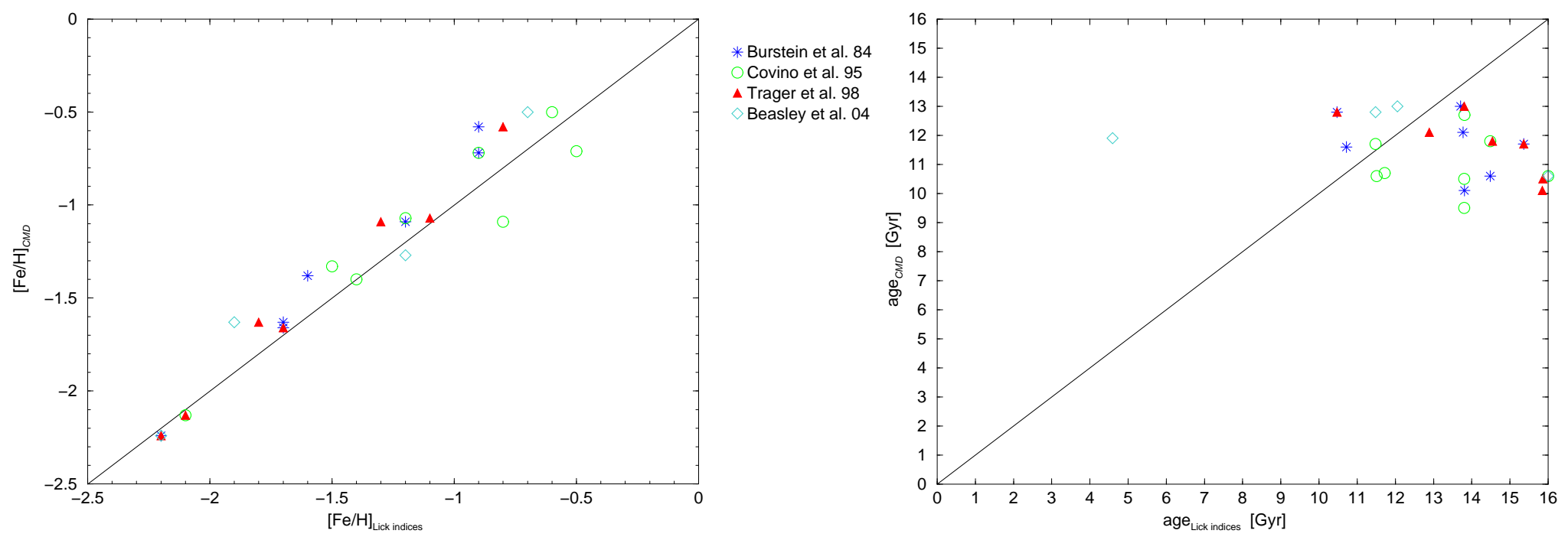

Fig. 3.4: Galactic GC observations: Metallicities (left) and ages (right) determined using our Lick index analysis tool (x-axis, using all measured indices available) vs. metallicities and ages determined by CMD analyses (y-axis, taken from Salaris \& Weiss 2002). Note that only results with model uncertainties of $\sigma($ age $) \leq 5$ Gyr are plotted. 

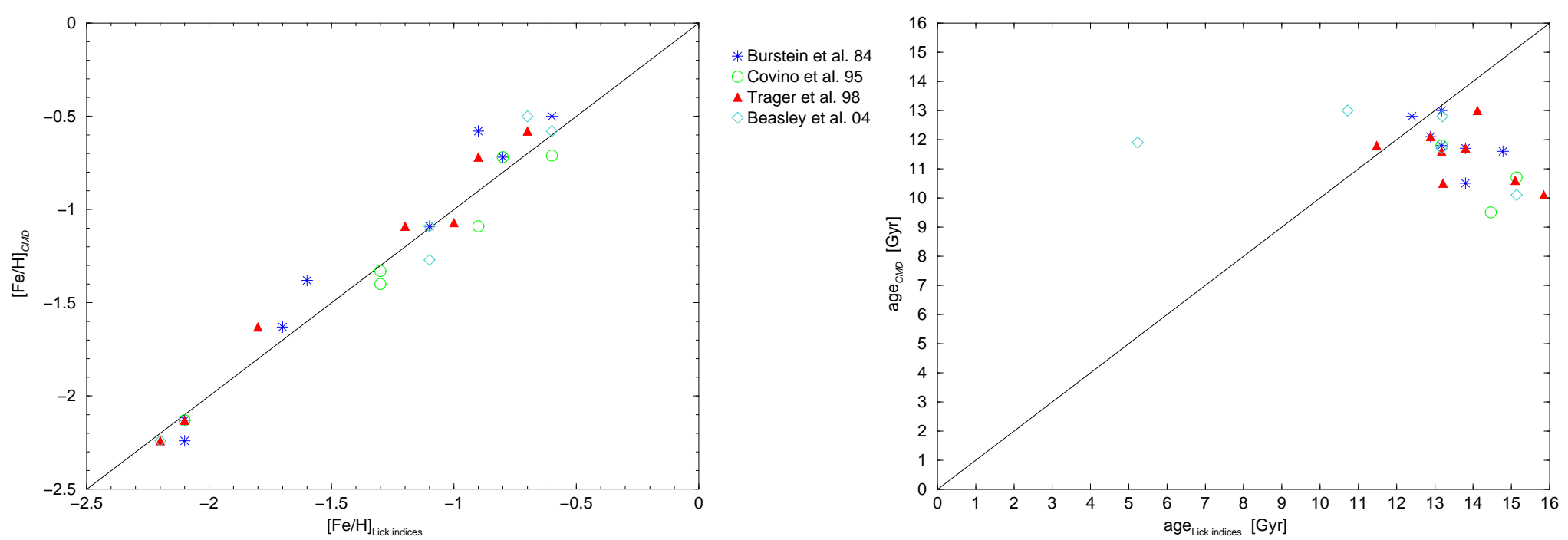

Fig. 3.5: Same as Fig. 3.4, but using metallicity-sensitive indices $\mathrm{Mg}_{1}, \mathrm{NaD}$, $[\mathrm{MgFe}]$, plus age-sensitive index $\mathrm{H} \beta$ as input only (left), and using age-sensitive indices $\mathrm{Ca} 4227, \mathrm{G} 4300, \mathrm{H} \beta$, and $\mathrm{TiO}_{1}$ as input only (right). 
Table 3.4: Mean ages and standard deviations of cluster ages determined using the Lick index analysis tool and CMD analysis (Salaris \& Weiss 2002), respectively, as shown in Figs. 3.4 and 3.5. Note that the values are computed without cluster NGC 6121.

\begin{tabular}{ccccc}
\hline \hline & \multicolumn{2}{c}{ all indices } & \multicolumn{2}{c}{ age-sensitive indices } \\
& $<$ age $>$ & \pm & $<$ age $>$ & \pm \\
\hline Lick-Analysis & 13.49 & 1.80 & 13.58 & 1.22 \\
CMD-Analysis & 11.54 & 1.08 & 11.57 & 1.04 \\
\hline
\end{tabular}

The agreement between $[\mathrm{Fe} / \mathrm{H}]$ obtained from our Lick index analysis tool and the corresponding values from CMD analyses is very good, with $\Delta[\mathrm{Fe} / \mathrm{H}]$ $\leq 0.3$ dex when using all available indices, and $\Delta[\mathrm{Fe} / \mathrm{H}] \leq 0.2$ dex when using mainly metal-sensitive indices. With one exception, the age determinations are relatively homogeneous, though the mean age obtained from index analyses is about 2 Gyrs too high compared to the results from CMD analyses. Table 3.4 gives the mean ages and standard deviations of clusters determined using the Lick index analysis tool and from CMD analyses, respectively. It shows that, using all available indices, not only the mean ages but also the age spreads are too high; most likely, this is due to varying horizontal branch $(\mathrm{HB})$ morphologies (see below). However, if only age-sensitive indices are used, the age spread is of the same magnitude than that obtained by CMD analyses.

As an example, Fig. 3.6 (top) shows the "best-fitting" model for the Galactic GC M3 (NGC 5272), together with the index measurements of Trager et al. (1998) used for the analysis. The best model has an age of $12.88\left(\begin{array}{l}-1.99 \\ +1.75\end{array}\right)$ Gyr and a metallicity of $[\mathrm{Fe} / \mathrm{H}]=-1.7( \pm 0)$ dex; compared with an age of $12.1( \pm 0.7)$ Gyr and $[\mathrm{Fe} / \mathrm{H}]=-1.66$ dex given by CMD analysis, this is a very good solution. We also give the $\pm 1 \sigma$ confidence intervals of our best model in terms of index values for SSPs with age $12.89-1.99=10.90 \mathrm{Gyr}$ and $12.89+1.74=14.63$ Gyr, respectively, and metallicity $[\mathrm{Fe} / \mathrm{H}]=-1.7$.

As seen in Figs. 3.4 and 3.5, most Galactic GCs are recovered very well in their metallicities by our Lick index analysis tool, in particular when the analysis is concentrated on the set of metal-sensitive indices $\mathrm{Mg}_{1}, \mathrm{NaD},[\mathrm{MgFe}]$, plus age-sensitive index $\mathrm{H} \beta$. The origin of the $\sim 2$ Gyr systematic difference between index-determined and CMD-based ages, as well as of the wider age spread we find is, most likely, due to the HB morphologies of the clusters. The Padova isochrones we use for the analyses have very red HBs over most of the parameter space; they have blue HBs only for metallicities $[\mathrm{Fe} / \mathrm{H}] \leq-1.7$ and ages higher than about 12 Gyr. Therefore, the age of an observed cluster with blue HB can possibly be underestimated by several Gyrs. Proctor et al. (2004), who use a similar technique to the one applied here, also find ages too high compared to values from CMD analyses; depending on the applied SSP models, they find mean ages of $13.1( \pm 2.3), 12.2( \pm 3.3)$, and $12.7( \pm 1.9)$ Gyr, respectively (cf. Table 3.4). We plan to analyse the influence of HB morphology on Lick index-based age determinations in a separate paper.

Interestingly, and despite the fact that the Lick index measurements used here have very different ages and qualities, the results are of comparable quality for each data set. E.g., the indices taken from Trager et al. (1998) are mea- 

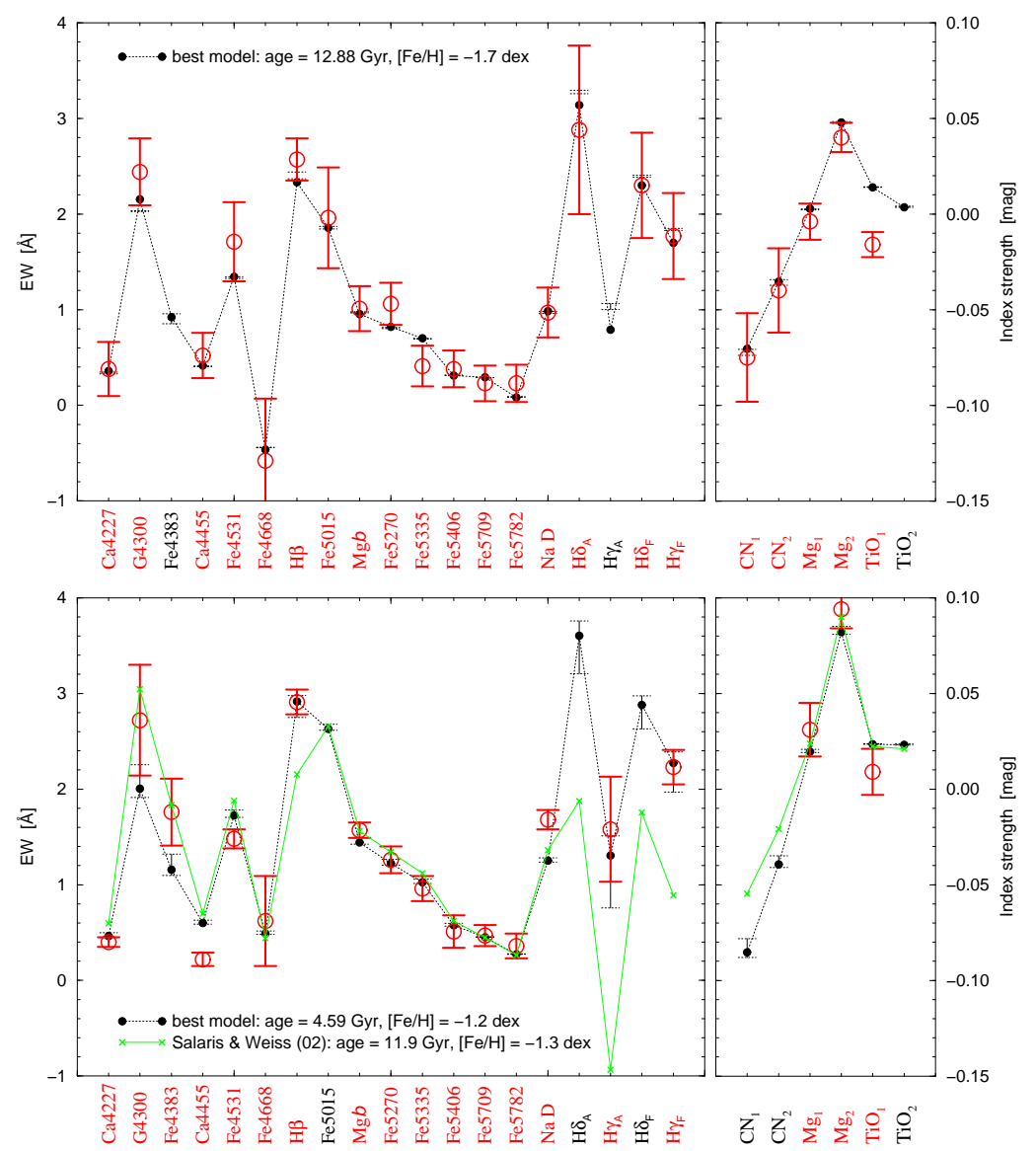

Fig. 3.6: Top: Lick index measurements of the Galactic GC M3 (NGC 5272) by Trager et al. (1998) with observational errors (open circles) and "best model" indices with the $\pm 1 \sigma$ confidence intervals (black dots). The best model has an age of $12.88\left(\begin{array}{c}-1.99 \\ +1.75\end{array}\right)$ Gyr and $[\mathrm{Fe} / \mathrm{H}]=-1.7( \pm 0)$ dex; Salaris \& Weiss $(2002)$ give age $=12.1( \pm 0.7)$ Gyr and $[\mathrm{Fe} / \mathrm{H}]=-1.66$ dex.

Bottom: Lick index measurements of the Galactic GC M4 (NGC 6121) by Beasley et al. (2004) with observational errors (open circles), and "best model" indices with the $\pm 1 \sigma$ confidence intervals (black dots). The best model has an age of only $4.59\left(\begin{array}{c}-0.31 \\ +0.80\end{array}\right)$ Gyr and $[\mathrm{Fe} / \mathrm{H}]=-1.2\left(\begin{array}{c}-0.1 \\ +0.0\end{array}\right)$ dex; Salaris \& Weiss $(2002)$ give age $=11.9( \pm 1.1)$ Gyr and $[\mathrm{Fe} / \mathrm{H}]=-1.27$ dex. Additionally, we plot model indices for the Salaris \& Weiss (2002) solution, i.e., an 11.9 Gyr / $[\mathrm{Fe} / \mathrm{H}]=-1.3$ dex SSP model (small crosses).

sured using the same original Lick-spectra as the Burstein et al. (1984) data set; however, the spectra were recalibrated, and more indices were measured. Nonetheless, the results from both data sets are comparable.

Though most results are acceptable, one cluster of our set is seriously misdetermined in terms of age. For the Galactic GC M4 (NGC 6121) the Lick 
index analysis tool gives an age of only $\sim 5$ Gyr (with a $1 \sigma$ uncertainty of less than 1 Gyr) using both all and only age-sensitive indices, while CMD analysis gives more than twice the age. Since the cluster does not have a very blue HB (Harris 1996 gives an HB ratio of nearly zero), we do not have a reasonable explanation for this. However, anomalies have been found for this cluster, and some properties are still being discussed in the literature (see, e.g., Richer et al. 2004 and references therein). Figure 3.6 (bottom) shows models for this "misdetermined" cluster: Together with the index measurements taken from Beasley et al. (2004), we show the index values for our best model (i.e., indices for a $\mathrm{SSP}$ with age $=4.59\left(\begin{array}{c}-0.31 \\ +0.80\end{array}\right) \mathrm{Gyr}$ and $\left.[\mathrm{Fe} / \mathrm{H}]=-1.2\left(\begin{array}{c}-0.1 \\ +0.0\end{array}\right) \mathrm{dex}\right)$, as well as for a model SSP using the Salaris \& Weiss (2002) solution (age $=11.9( \pm 1.1)$ Gyr and $[\mathrm{Fe} / \mathrm{H}]=-1.3 \mathrm{dex})$. The indices that differ most between the two models (and for which measurements are available) are G4300, Fe4383, and the Balmer line indices $\mathrm{H} \beta$ and $\mathrm{H} \gamma$; remarkably, the Balmer lines seem to be completely responsible for the misdetermination.

\subsubsection{Examples and tests II: M31 GCs and non-solar abun- dance ratios}

Since for Andromeda galaxy (M31) GCs it is, unfortunately, not possible to obtain high-quality colour magnitude diagrams, reliable determinations of age and metallicity that could be used as "default values" for comparisions are not available. Therefore, for M31 GCs we can only compare our Lick indexbased determinations with results taken from the literature, which are based on spectral indices themselves.

For our analyses, we use the Lick index measurements of M31 GCs presented by Beasley et al. (2004); while not presenting their own age or metallicity determinations for individual clusters, they distinguish four classes for their sample of cluster candidates: Young, intermediate age, and "normal" old GCs. Additionally, some sources are suspected to be foreground galaxies. Beasley et al. have measured all available Lick indices with the exception of $\mathrm{TiO}_{2}$.

In Fig. 3.7, we compare our metallicity determinations using the Lick index analysis tool with results presented by Barmby et al. (2000) (top left panel) and Puzia et al. (2005) (top right panel). While Barmby et al. use calibrations given by Brodie \& Huchra (1990) for their spectroscopic metallicity determinations, using their own measurements of absorption line indices, Puzia et al. (2005) use an $\chi^{2}$ approach using Lick index models from Thomas et al. (2003, 2004), which account for non-solar abundance ratios. Puzia et al. use the same database as we do (i.e., the Lick index measurements published by Beasley et al. 2004). Instead of $[\mathrm{Fe} / \mathrm{H}]$, they give total metallicities $[\mathrm{Z} / \mathrm{H}]$; however, according to Thomas et al. (2003), $[\mathrm{Fe} / \mathrm{H}]$ in the ZW84 scale is in excellent agreement with $[\mathrm{Z} / \mathrm{H}]$. Hence, our results, given in $[\mathrm{Fe} / \mathrm{H}]$, are perfectly comparable to the Puzia et al. results and are appropriate for testing for the influence of non-solar abundance ratios in our results. For both the Barmby et al. (2000) and Puzia et al. (2005) metallicity determinations, we find good agreement with our results. Only for clusters that are classified as young (i.e., age $\leq 1-2$ Gyr) do we find relatively large differences in $[\mathrm{Fe} / \mathrm{H}]$; however, this reflects our expectations, since the models are calibrated using mainly intermediate-age or old Galactic stars. 

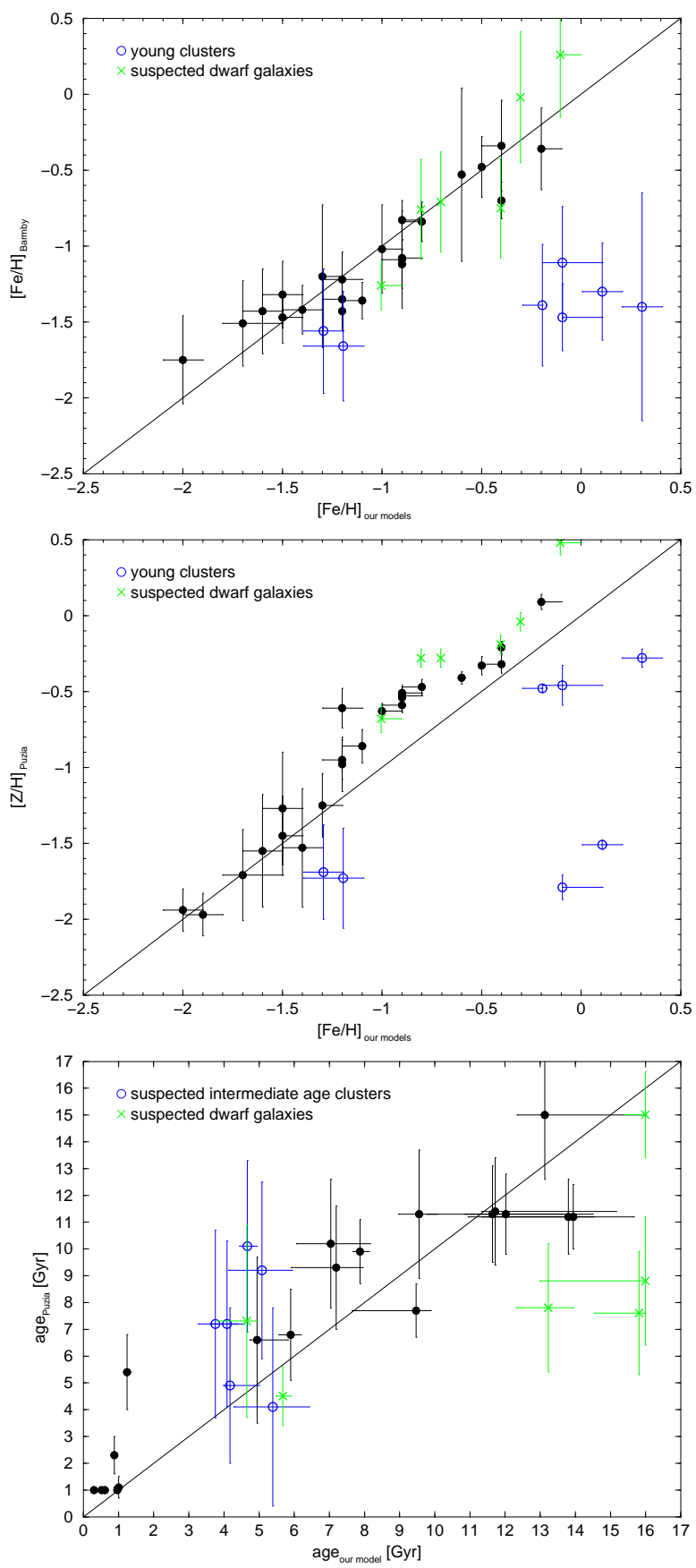

Fig. 3.7: M31 GCs: Metallicities and ages for the Beasley et al. (2004) GC sample, determined using our Lick index analysis tool (x-axis, using all measured indices available) vs. metallicity determinations taken from Barmby et al. (2000) (top panel) and Puzia et al. (2005) (middle panel), and vs. age determinations taken from Puzia et al. (2005) (bottom panel). The classification as "young cluster" and "suspected dwarf galaxy" is taken from Beasley et al. (2004). 

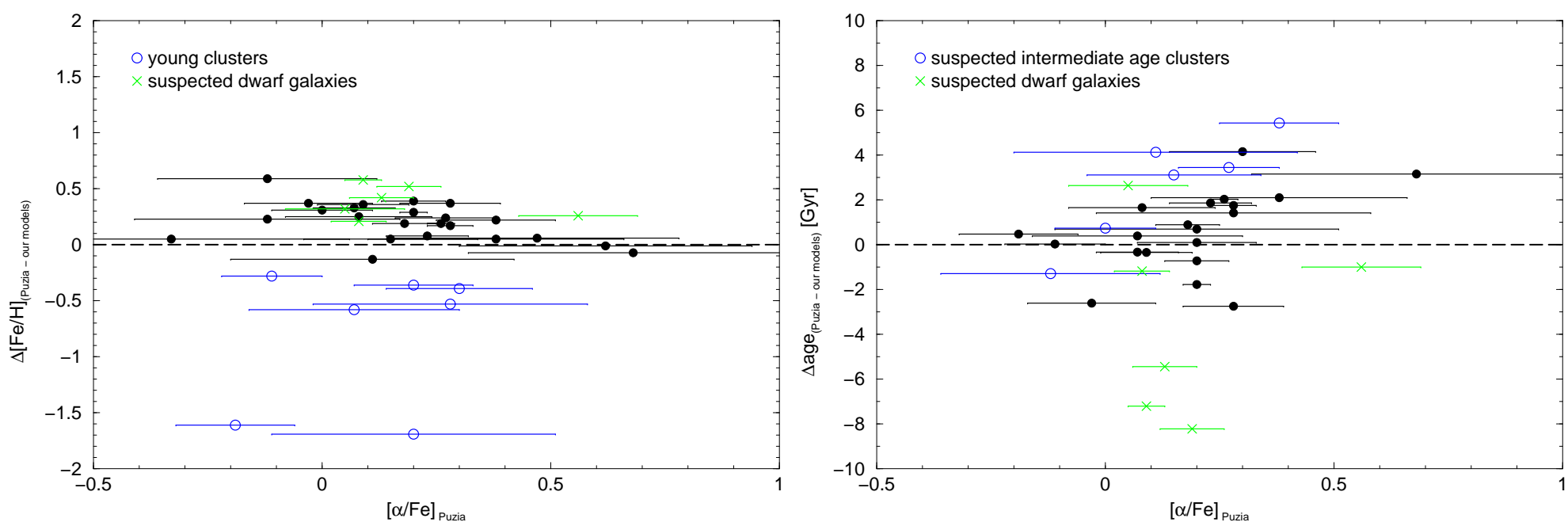

Fig. 3.8: M31 GCs: Absolute differences between parameters derived using our Lick index analysis tool and results from Puzia et al. (2005), against $[\alpha / \mathrm{Fe}]$ taken from Puzia et al. (2005). Left: $\left([\mathrm{Z} / \mathrm{H}]_{\text {Puzia }}-[\mathrm{Fe} / \mathrm{H}]_{\text {our models }}\right)$; Right: (age Puzia - age $\left._{\text {our models }}\right)$. The classification as "intermediate-age cluster" and "suspected dwarf galaxy" is taken from Beasley et al. (2004). 
In the bottom panel of Fig. 3.7, we compare our results with ages determined by Puzia et al. (2005). Again, the results are in surprisingly good agreement, if sources suspected of being foreground dwarf galaxies are not considered. For the set of intermediate-age clusters identified by Beasley et al. (2004), our results reflect this classification perfectly.

Compared with the classification of Beasley et al. (2004), the largest disagreements in both age and metallicity occur for young clusters and for suspected dwarf galaxies; it is no surprise that models computed to fit GCs are not appropriate to galaxies (and, therefore, different methods lead to different results).

Since Puzia et al. (2005) also determine $\alpha$-enhancements for the GC sample, we can check for possible systematic offsets of our determinations compared to theirs due to non-solar abundance ratios.

In Fig. 3.8, absolute differences between metallicities (left panel) and ages (right panel) derived using our models and from Puzia et al. (2005) are plotted against $[\alpha / \mathrm{Fe}]$. Relatively surprising is that no general trend for the differences in both age and metallicity determinations with $\alpha$-enhancement can be observed, if the large error bars of the $[\alpha / \mathrm{Fe}]$ determinations are taken into account. Hence, the slight offset between metallicities determined by Puzia et al. and by us (cf. Fig. 3.7, top right panel) for $[\mathrm{Fe} / \mathrm{H}]$ larger than $\sim-1.2$ dex seems not to be due to the use of solar-scaled against $\alpha$-enhanced models.

\subsection{Summary and outlook}

To cope with the observational progress that makes star cluster \& globular cluster spectra accessible in a wide variety of external galaxies, we have computed a large grid of evolutionary synthesis models for simple stellar populations, including 25 Lick/IDS indices using the empirical calibrations of Worthey et al. (1994) and Worthey \& Ottaviani (1997). Comparison of the SSP models with Galactic GC observations shows good agreement between models and data.

We find that the well-known and widely used age-sensitive indices $\mathrm{H} \delta_{A}$ and $\mathrm{H} \gamma_{A}$ also show a strong metallicity dependence. The "metallicity sensitivity parameter" S introduced by Worthey (1994) for old stellar populations with solar metallicity is reproduced well by our models. Our models allow us to extend this concept to younger ages and non-solar metallicities. We find the sensitivity of different indices with respect to age and metallicity to depend on age and metallicity; e.g., all indices are generally more age sensitive at low than at high metallicity. Another important issue is the absolute difference in index strength for varying age or metallicity: Due to the limited accuracy of any index measurement, these absolute differences in practice can be of greater importance than the sensitivity given by $\mathrm{S}$.

We present a new advanced tool for interpreting absorption-line indices, the Lick index analysis tool LINO. Following an $\chi^{2}$ - approach, this tool determines age and metallicity, including their respective $\pm 1 \sigma$ uncertainties, using all, or any subset of, measured indices. Testing our tool against index measurements from various authors for Galactic GCs, which have reliable age and metallicity determinations from CMD analyses in the literature, shows very good agreement: Metallicities of GCs are recovered to \pm 0.2 dex using 6 appropriate indices alone $\left(\mathrm{Mg}_{1}, \mathrm{Mg} b, \mathrm{Fe} 5270\right.$, Fe5335, $\left.\mathrm{NaD}, \mathrm{H} \beta\right)$. Age determinations from 
Lick indices consistently yield ages $\sim 2$ Gyr higher than those obtained from CMDs. The origin of this discrepancy is not yet understood. Index measurements for M31 clusters are analysed and compared to results from the literature, and a good agreement between our results and age and metallicity determinations from the literature is found. We show that the drawback of not having non-solar abundance ratio models does not seriously affect our results.

We will apply LINO to the interpretation of intermediate-age and old GC populations in external galaxies, complementing our SED analysis tool for the interpretation of broad-band spectral energy distributions.

All models are accessible from our website ${ }^{8}$.

\subsection{References}

Anders P. and Fritze - v. Alvensleben U., 2003, A\&A 401, 1063

Anders P., Bissantz N., Fritze - v. Alvensleben U. and de Grijs R., 2004, MNRAS 347, 196

Barmby P., Huchra J.P., Brodie J.P., et al. , 2000, AJ 119, 727

Beasley M.A., Brodie J.P., Strader J., et al. , 2004, AJ 128, 1623

Bertelli G., Bressan A., Chiosi C., Fagotto F. and Nasi E., 1994, A\&AS 106, 275

Bicker J., Fritze - v. Alvensleben U., Möller C.S. and Fricke K.J., 2004, A\&A 413,37

Brodie J.P. and Huchra J.P., 1990, ApJ 362, 503

Burstein D., Faber S.M., Gaskell C.M. and Krumm N., 1984, ApJ 287, 586

Chabrier G. and Baraffe I., 1997, A\&A 327, 1039

Covino S., Galletti S. and Pasinetti L.E., 1995, A\&A 303, 79

Edvardsson B., Andersen J., Gustafsson B., et al. , 1993, A\&A 275, 101

Girardi L., Bressan A., Chiosi C., Bertelli G. and Nasi E., 1996, A\&AS 117, 113

Gorgas J., Cardiel N., Pedraz S. and González J.J., 1999, A\&AS 139, 29

Harris W.E., 1996, AJ 112, 1487

Harris G.L.H., Harris W.E. and Pool G.B., 1999, AJ 117, 855

Harris G.L.H. and Harris W.E., 2000, AJ 120, 2423

Harris W.E. and Harris G.L.H., 2002, AJ 123, 3108

Kim Y.-C., Demarque P., Yi S.K. and Alexander D.R., 2002, ApJS 143, 499

Kissler-Patig M., 2000, RvMA 13, 13

Kuntschner H., Ziegler B.L., Sharples R.M., Worthey G. and Fricke K.J., 2002, A\&A 395, 761

Kurth O.M., Fritze - v. Alvensleben U. and Fricke K.J., 1999, A\&AS 138, 19

Lejeune T., Cuisinier F. and Buser R., 1997, A\&AS 125, 229

Lejeune T., Cuisinier F. and Buser R., 1998, A\&AS 130, 65

\footnotetext{
${ }^{8}$ http://www.astro.physik. uni-goettingen.de/ galev/
} 
Lilly T., 2003, Master's thesis, University Observatory Göttingen (Germany)

Maraston C., Greggio L., Renzini A., et al. , 2003, A\&A 400, 823

Pagel B.E.J. and Tautvaišienè G., 1995, MNRAS 276, 505

Puzia T.H., Saglia R.P., Kissler-Patig M., et al. , 2002, A\&A 395, 45

Puzia T.H., Perrett K.M. and Bridges T.J., 2005, A\&A 434, 909

Richer H.B., Fahlmann G.G., Brewer J., et al. , 2004, AJ 127, 2771

Salaris M. and Weiss A., 2002, A\&A 388, 492

Salasnich B., Girardi L., Weiss A. and Chiosi C., 2000, A\&A 361, 1023

Salpeter E.E., 1955, ApJ 121, 161

Schulz J., Fritze - v. Alvensleben U., Möller C.S. and Fricke K.J., 2002, A\&A 392,1

Thomas D. and Maraston C., 2003, A\&A 401, 429

Thomas D., Maraston C. and Bender R., 2003, MNRAS 339, 897

Thomas D., Maraston C. and Korn A., 2004, MNRAS 351, 19

Trager S.C., Worthey G., Faber S.M., Burstein D. and González J.J., 1998, ApJS 116, 1

Trager S.C., Faber S.M., Worthey G. and González J.J., 2000a, AJ 119, 1645

Trager S.C., Faber S.M., Worthey G. and González J.J., 2000b, AJ 120, 165

Tripicco M.J. and Bell R.A., 1995, AJ 110, 3035

Worthey G., 1994, ApJS 95, 107

Worthey G., Faber S.M., González J.J. and Burstein D., 1994, ApJS 94, 687

Worthey G. and Ottaviani D.L., 1997, ApJS 111, 377

Worthey G., 2004 (private communication)

Zepf S., 1999, AAS 194, 4015

Zepf S., 2002, IAUS 207, 653 


\section{Chapter 4}

\section{Analysing globular cluster observations II: Combining broad-band SED and Lick index analysis ${ }^{1}$}

\subsection{Introduction}

An independent determination of ages and metallicities of globular clusters (GCs) is essential for studies of globular cluster systems (GCSs) and in attempts to derive clues to the formation history of their parent galaxy from its GCs. However, even when analysing Lick indices age-metallicity degeneracy remains a severe problem.

In this chapter, I present a new method for the determination of ages and metallicities of individual star clusters by combining the information inherent in broad-band colour and Lick index measurements, and I address the question of which kind of data is necessary, or most useful, to constrain ages and metallicities to best accuracy.

Using the well established spectral energy distribution analysis tool AnalySED, it is possible to obtain ages, metallicities, and masses of individual GCs by comparing multi-band photometric observations with an extensive grid of SSP model SEDs. This is done in a statistically advanced way, on the basis of a $\chi^{2}$-approach. However, since for all colours the evolution slows down considerably at ages older than about $8 \mathrm{Gyr}$, even with several passbands and a long wavelength basis the results become more and more uncertain for older clusters. Therefore, I incorporated empirical calibrations for Lick indices into the models and developed a Lick indices analysis tool that works in the same way as the SED analysis tool. However, even when using spectral information, results still suffer from age-metallicity degeneracies: While metallicities from the Lick index analysis proved to be very reliable, 1 sigma uncertainties in ages can still be very high (up to $~ 10 \mathrm{Gyr}$ ). In order to further reduce the degeneracies inherent in each kind of integrated light data (broad-band SEDs and Lick indices),

\footnotetext{
${ }^{1}$ After minor changes, this chapter will be submitted shortly to be published in A\&A.
} 
I have developed a new method (PRODUCT) to combine the analysis of both methods (AnalySED and Lick-Analysis) and, hence, to exploit all the available information simultaneously in a mathematically reasonable way.

\subsection{Models}

GALEV evolutionary synthesis models describe the spectrophotometric evolution of the integrated light of large stellar populations like star clusters or galaxies from the onset of star formation (SF) over a Hubble time.

Input physics include the theoretical spectral library from Lejeune et al. $(1997,1998)$ as well as theoretical isochrones from the Padova group for $Z=0.0004$, 0.004, 0.008, 0.02 and 0.05 (cf. Bertelli et al. 1994), and, so far only implemented for our models of Lick indices, for $Z=0.0001$ (cf. Girardi et al. 1996); recent versions of these isochrones include the TP-AGB phase of stellar evolution which is important for intermediate age stellar populations (cf. Schulz et al. 2002). We assume a standard Salpeter (1955) initial mass function (IMF) from 0.15 to about $70 \mathrm{M}_{\odot}$; lowest mass stars $\left(\mathrm{M}_{\odot}<0.6\right)$ are taken from Chabrier and Baraffe (1997) (cf. Schulz et al. 2002 for details). Lick indices are computed employing the empirical polynomial fitting functions of Worthey et al. (1994) and Worthey \& Ottaviani (1997), which give Lick index strengths of individual stars as a function of their effective temperature $T_{\text {eff }}$, surface gravity $g$, and metallicity $[\mathrm{Fe} / \mathrm{H}]$.

For descriptions of all aspects of GALEV and its input physics see Schulz et al. (2002), Anders \& Fritze-v. Alvensleben (2003), Bicker et al. (2004), and Lilly \& Fritze-v. Alvensleben (2006). Throughout this paper, we describe the metallicity $Z$ by $[\mathrm{Fe} / \mathrm{H}]$ and define $[\mathrm{Fe} / \mathrm{H}]:=\log \left(Z / Z_{\odot}\right)$.

For the purpose of this paper, we have computed a large grid of Single Stellar Population (SSP) models consisting of 22 metallicities $(-1.7 \leq[\mathrm{Fe} / \mathrm{H}] \leq 0.4$, in steps of $0.1 \mathrm{dex}$ ) and 4000 ages from 4 Myr to 16 Gyr in steps of 4 Myr; the additional metallicities are computed by linear interpolation in $[\mathrm{Fe} / \mathrm{H}]$ between the 5 metallicities given above. Each point of the model grid then consists of 25 Lick indices as well as of broad-band Spectral Energy Distributions in a variety of filter systems, ranging from UV to the NIR.

\subsection{Analysis tools}

In this section, after giving an overview of our well-established tools for analysing broad-band colours (AnalySED, Anders et al. 2004a,b) and spectral indices (Lick Analysis Tool, Lilly \& Fritze-v. Alvensleben 2006), we present our method to combine the analysis of different data sets like SEDs in different photometric systems or SEDs and Lick indices (PRODUCT), and we discuss the reliability of our new method.

\subsubsection{AnalySED and Lick Analysis Tool}

All our analysis tools are based on a mathematically reliable $\chi^{2}$ technique: Observational data, like colours or spectral indices, are compared with a large grid 
of SSP models in order to constrain ranges of allowed parameter combinations (here: age and metallicity) for individual star clusters.

For this purpose, a probability is assigned to each point of the model grid:

$$
p(n) \propto\left(-\chi^{2}\right)
$$

where

$$
\chi^{2}=\sum \frac{\left(m_{\text {obs }}-m_{\text {model }}\right)^{2}}{\sigma_{\text {obs }}^{2}+\sigma_{\text {model }}^{2}}
$$

with $m_{\text {obs }}$ and $m_{\text {model }}$ being the observed and the model magnitudes, or indices, respectively, and $\sigma_{\mathrm{obs}}$ and $\sigma_{\text {model }}$ being the respective uncertainties.

Subsequently, the model with the highest probability is chosen as the "bestfit model". $\pm 1 \sigma$ confidence intervals are determined by summing up models with decreasing probabilities until 68 per cent total probability are reached.

Anders et al. (2004) used this method for the purpose of analysing broadband colours of star clusters. His analysis tool AnalySED compares cluster SEDs with a large grid of SSP models in order to independently determine ages, metallicities, (internal) extinctions and masses of individual clusters. He obtained good results for young (age < 1 Gyr) clusters; however, results turned out to be severely uncertain for old globular clusters (GCs).

Therefore, Lilly \& Fritze-v. Alvensleben (2006) extended the code to describe and analyse Lick indices. With these new models and the respective Lick-index tool we are now able to independently determine ages and metallicities of individual clusters to much higher precision than was possible by analysing broad-band SEDs: As compared to CMD analyses, we got very accurate metallicities over the full range of ages (cf. Lilly \& Fritze-v. Alvensleben 2006, Fig. 4). However, especially in view of accurate age determinations, age-metallicity degeneracy still remains a severe problem.

\subsubsection{PRODUCT}

Our $\chi^{2}$-technique deals with the absolute numerical differences between model and observed data, weighted only by both observational and model uncertainties. Therefore, since broad-band colours and Lick indices are very different types of data (measured in different units and reaching different ranges of numerical values), it is no possible to just put all available data (i.e. broad-band SEDs and Lick indices, or even SEDs in two different filter systems, e.g. a broad-band and an intermediate or narrow-band system) in one model grid and proceed as usual.

To be able to exploit all the information available for a given set of star clusters, we first analyse each data set separately, using the appropriate analysis tool (AnalySED or the Lick Analysis Tool). During these processes two independent probabilities are assigned to each model grid point, resulting in two probability spaces (one for each dataset). These probabilities (i.e., the complete probability spaces) can be multiplied. After renormalization of the resulting probability space, we can determine the best solution again following the same technique as described above.

Following this method, it is possible to analyse "simultaneously" any compilation of independent and possibly complementary datasets. However, it is 
important to realize that the final result of an analysis using two (or more) different datasets is mainly determined by the dataset which gives the bestconstrained solution, i.e. for which the probability space is characterized by a relatively well defined "peak", at least in contrast to what is obtained for the other dataset. In the majority of cases, this "better" dataset is the one with the Lick indices.

Therefore, PRODUCT is most useful in cases where sets of inhomogeneous, incomplete, or poorly observed (i.e., large errorbars) data are available (which in any case must be statistically independent), e.g. two different sets of broadband colours, or Lick indices which were determined from low S/N spectra not originally intended to be be used for Lick index measurements. We will show that, in this cases, using PRODUCT these data can still successfully be used for independent age and metallicity determinations.

\subsection{Examples and tests}

In this section, we demonstrate the performance of our new method by analysing star cluster samples for the LMC and for NGC 5128. These have, to some extent, broad-band photometric, Lick index measurements, and CMD-based independent age and metallicity determinations.

\subsubsection{LMC}

We have analysed a compilation of star clusters in the LMC, of intermediate age and old, for which both UBVJHKs photometry and a set of Lick indices are available. In most cases, CMD-based age determinations as well as reliable metallicity determinations are available in the literature.

Lick indices are taken from Beasley et al. (2002), SEDs from Bica et al. (1996) for UBV and from Pessev et al. (2006) for JHKs. Pessev et al. give observations for a variety of aperture diameters; to get a dataset as consistent as possible, we chose the observations with the same apperture diameters as used by Bica et al. (1996) for each cluster. The SEDs are dereddened following the extinction law given by Cardelli et al. (1989), using a value of $R_{V}=3.41$ appropriate for the LMC, as given by Gordon et al. (2003). We have used extinctions $A_{V}$ for individual clusters as listed in Pessev et al. (2006) which include not only Galactic foreground reddening but also internal reddening in the LMC.

To give an overview over the properties of the cluster system, in Fig. 4.5 we show the age-metallicity relation of our LMC cluster sample by plotting age vs. metallicity for literature based values (black dots; for references cf. Tabs. 4.1 through 4.4) as well as using our results for the combined analysis of UBVJHK photometry and the 5 indices $\mathrm{H} \beta, \mathrm{Mg} b, \mathrm{Mg}_{2}, \mathrm{Fe} 5270$, and Fe5335 using PRODUCT.

Taking into account that our parameter space is restricted to metallicities $[\mathrm{Fe} / \mathrm{H}] \geq-1.7 \mathrm{dex}$, and ages $\leq 16 \mathrm{Gyr}$, the literature based relation is reproduced reasonably well. However, one misdetermination is clearly visible in the plot (marked by a circle in the left panels of this as well as the other plots shown in this section). For this cluster, identified as NGC 1916, we determine an age of $6.36_{-1.37}^{+2.05} \mathrm{Gyr}$ which is much younger than the age given in the literature 

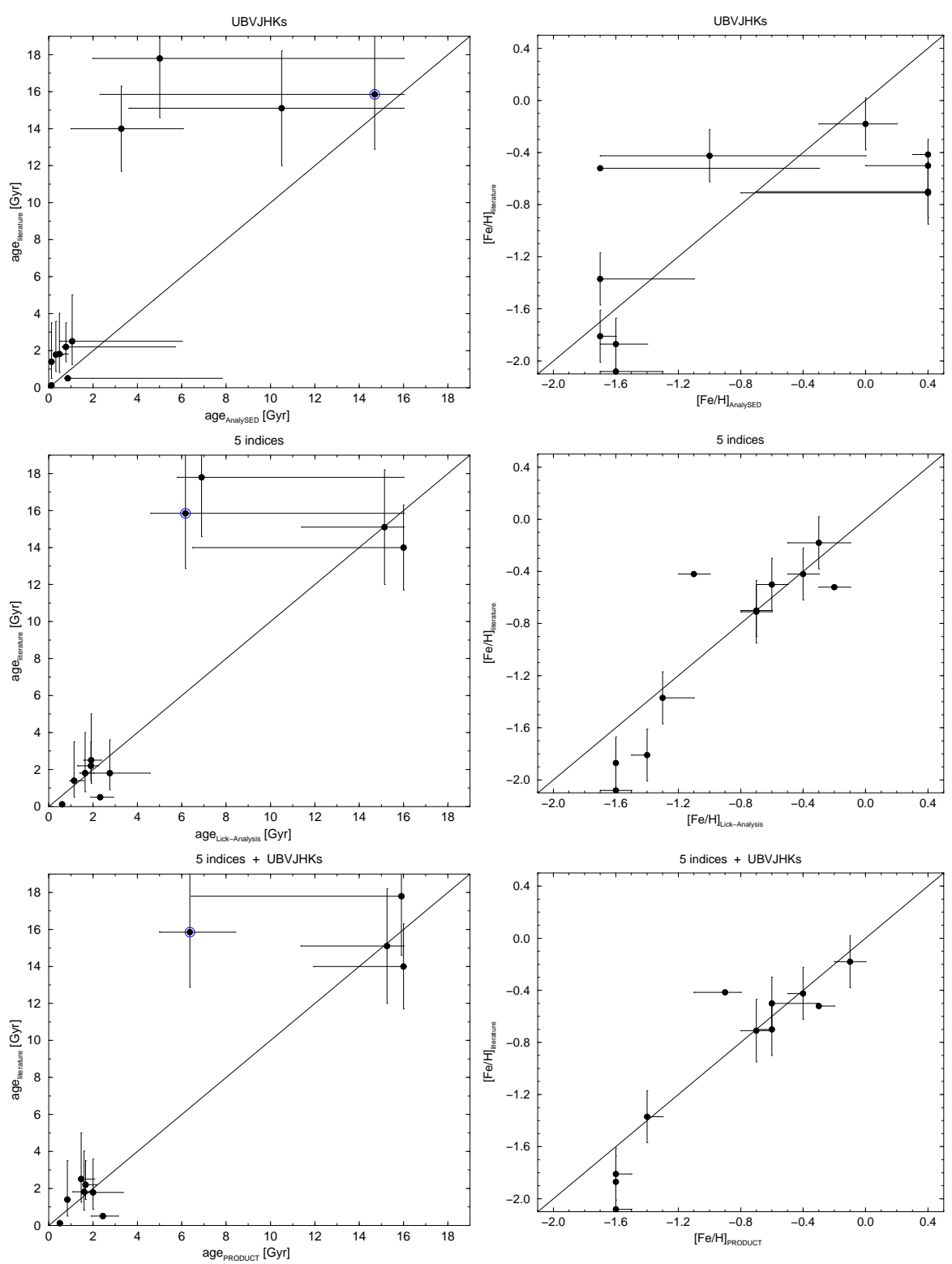

Fig. 4.1: Ages (left) and metallicities (right) for 11 LMC star clusters analysed using broad-band photometry UBVJHKs for AnalySED ( $t o p$ ), the 5 Lick indices $\mathrm{H} \beta, \mathrm{Mg} b, \mathrm{Mg}_{2}, \mathrm{Fe} 5270, \mathrm{Fe} 5335$ for the Lick analysis (middle), and PRODUCT using both sets of data (bottom), plotted against ages and metallicities given in the literature (y-axes). Note that the age given in the literature for cluster NGC 1916 (blue circle) is very unsure (see text). 

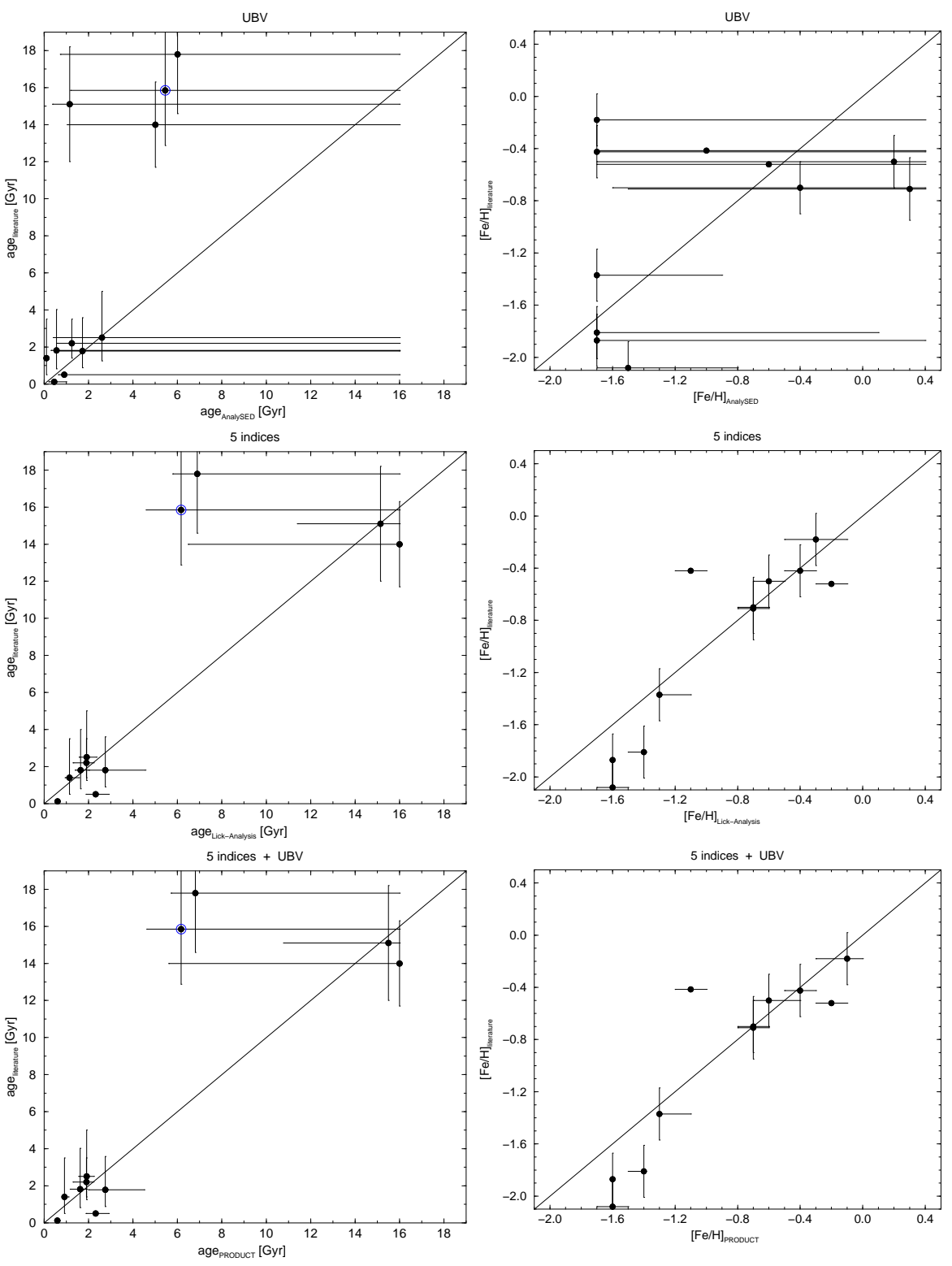

Fig. 4.2: Same as Fig. 4.1, but using UBV only for AnalySED. 

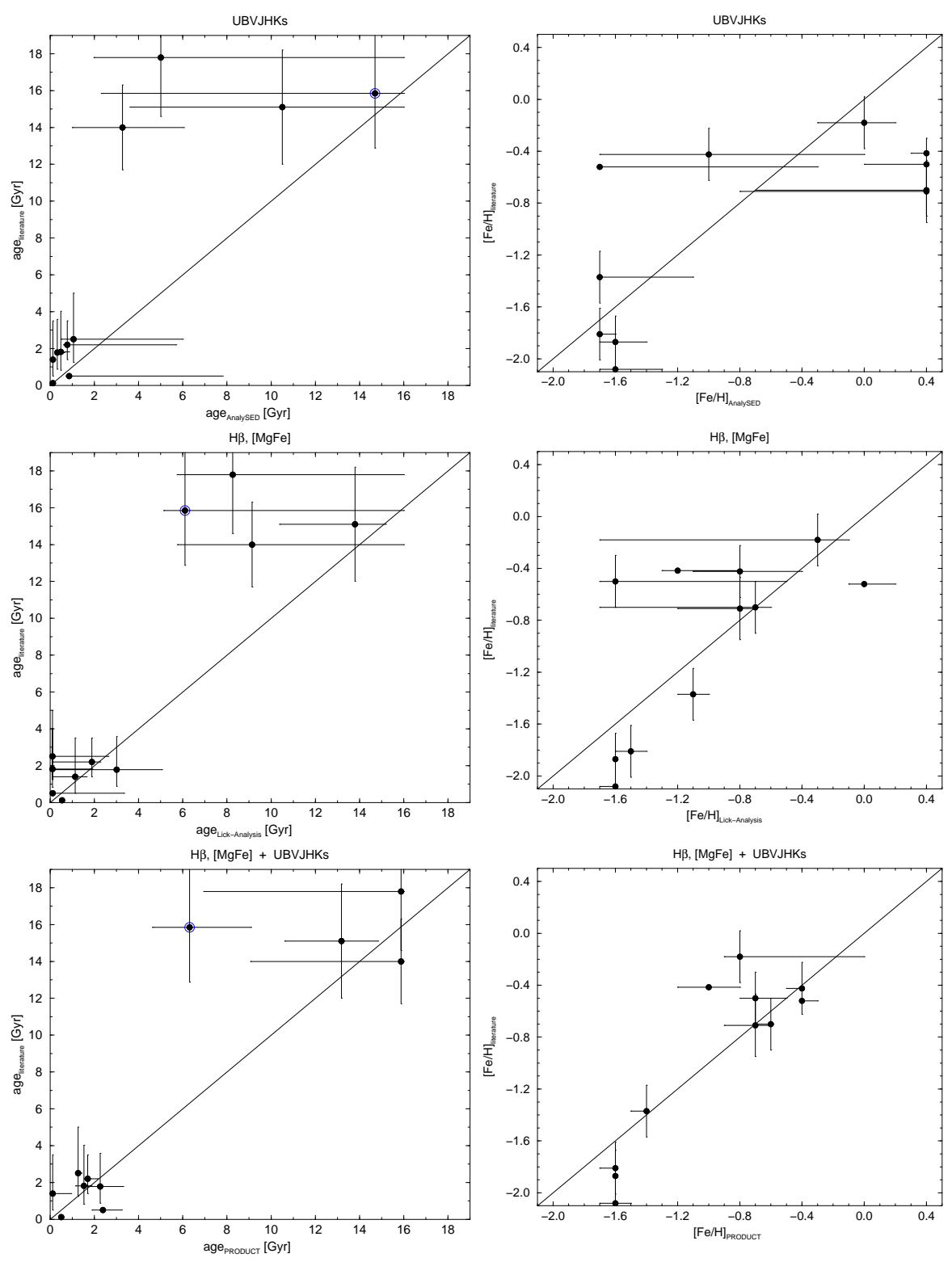

Fig. 4.3: Same as Fig. 4.1, but using Lick indices $\mathrm{H} \beta$ \& $[\mathrm{MgFe}]$ only for the Lick analysis. 

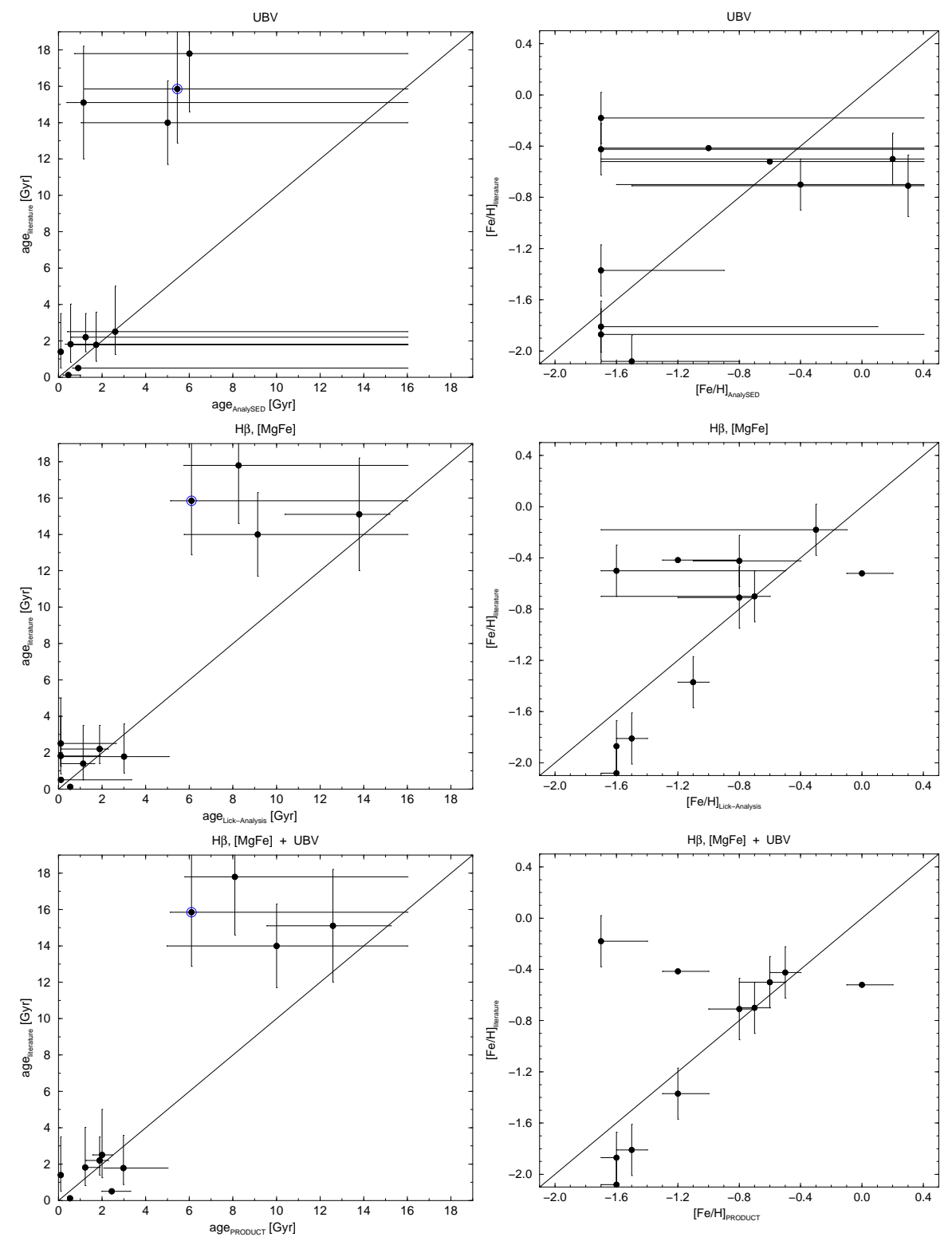

Fig. 4.4: Same as Fig. 4.1, but using UBV only for AnalySED, and $\mathrm{H} \beta$ \& $[\mathrm{MgFe}]$ only for the Lick analysis. 


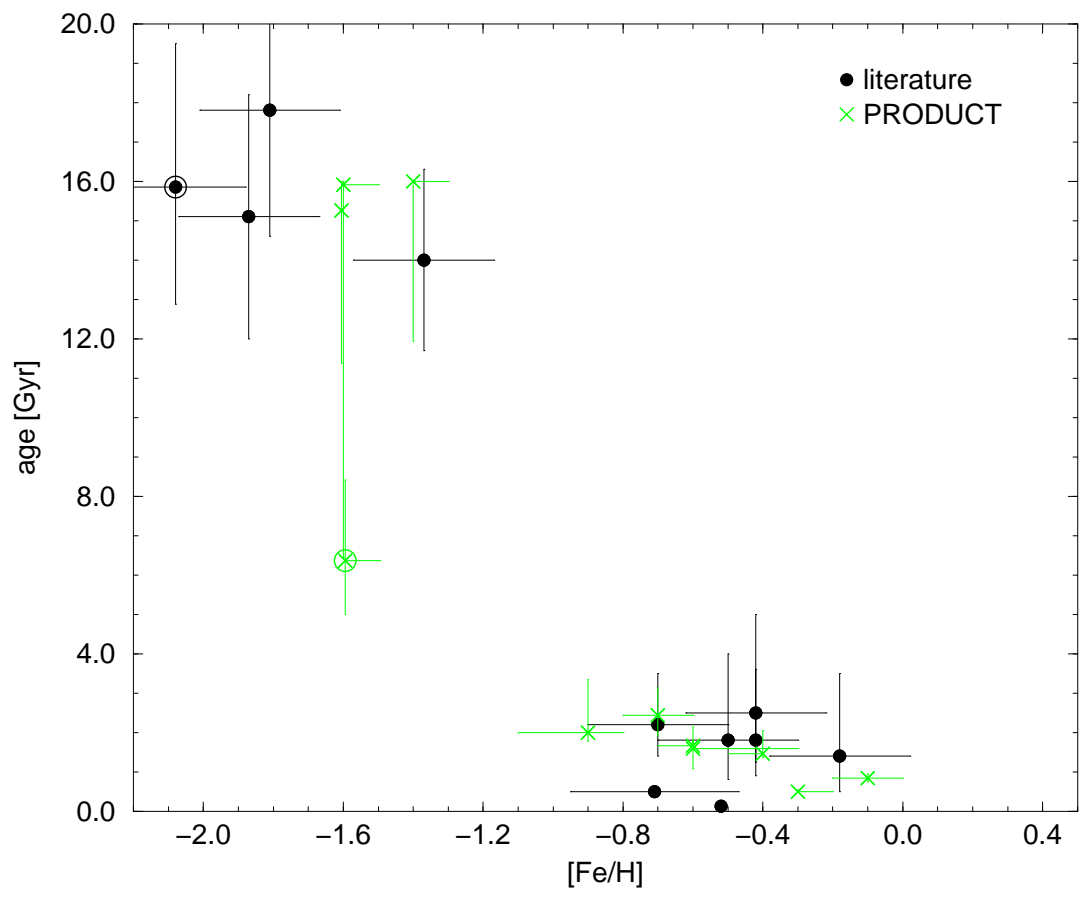

Fig. 4.5: Age-metallicity relation of our LMC cluster sample. Black dots give literature values, green crosses our best results obtained by analysing UBVJHK photometry together with the 5 Lick indices $\mathrm{H} \beta, \mathrm{Mg} b, \mathrm{Mg}_{2}, \mathrm{Fe} 5270$, and Fe5335 using PRODUCT. Cluster NGC 1916 is marked by a circle around the dot and the cross, respectively. 
$\left(15.85_{-2.97}^{+3.65} \mathrm{Gyr}\right)$. However, this age is not based on a reliable CMD analysis but by a visual examination of the CMD of this cluster which suffers from "serious differential reddening" (cf. Mackey \& Gilmore 2003). By deciding that, visually, the CMD does not differ too much from the CMDs of old clusters other than NGC 1916, the authors averaged the ages of these clusters to arrive at the above age estimate of 15.85 Gyr for NGC 1916.

To give the reader a feeling about which kind of observational data is most useful or necessary, respectively, to break the age-metallicity degeneracy inherent in all kind of integrated light data (especially for old star clusters) and to constrain age and metallicity with maximal accuracy but at the same time as efficient as possible (i.e., using not more observations/measurements as necessary), we have analysed not only the 5 indices and the full set of colours UBVJHK but also a variety of combinations of rich (i.e., many colours/indices) and poor (i.e., few colours/indices) datasets:

1. The 5 Lick indices $\mathrm{H} \beta, \mathrm{Mg} b, \mathrm{Mg}_{2}, \mathrm{Fe} 5270$, and Fe5335.

2. $\mathrm{H} \beta$ and $[\mathrm{MgFe}]^{2}$ only.

3. UBVJHKs photometry.

4. UBV photometry only.

All sets and the reasonable combinations (i.e., each rich/poor set of colours with the rich/poor sets of Lick indices) have been analysed using the appropriate analysis tool (AnalySED, Lick Analysis Tool, and, for the combined analysis of SEDs and indices, PRODUCT).

Our results in terms of best ages and metallicities, including their respective $\pm 1 \sigma$ confidence intervals, are plotted in Figs. 4.1 through 4.4 against the respective literature values; the exact numerical values of all our results together with ages and metallicities taken from the literature and the corresponding references are given in Tabs. 4.1 through 4.4 .

Analysing the rich colour set (UBVJHK), the results show a relative high degeneracy, exhibiting large error bars in both age and metallicity for most clusters despite the long baseline from $\mathrm{U}$ to $\mathrm{K}$ (Fig. 4.1, top panels). Interestingly, old clusters exhibit large error bars in ages but relatively small error bars in metallicities; intermediate age clusters behave vice versa (remember that, in the LMC, old clusters are metal-poor, young clusters metal-rich).

Analysing the set of 5 indices $\mathrm{H} \beta, \mathrm{Mg} b, \mathrm{Mg}_{2}, \mathrm{Fe} 5270$, and $\mathrm{Fe} 5335$, ages are relatively well constrained for intermediate age clusters, but old clusters still have high uncertainties in their ages (Fig. 4.1, middle panels). In terms of metallicities, however, the results are very good for the full range in ages and metallicities.

If the analysis of these two datasets is combined using PRODUCT, the age determinations for old clusters which were highly uncertain using photometry and indices alone, respectively, are very much improved (Fig. 4.1, bottom

\footnotetext{
${ }^{2}[\mathrm{MgFe}]$ is a combination of metal-sensitive indices that is known to be widely unaffected by non-solar abundance ratios (see, e.g., Thomas et al. 2003). It is defined as $[\mathrm{MgFe}]:=$ $\sqrt{<\mathrm{Fe}>\times \mathrm{Mg} b}$, with $<\mathrm{Fe}>:=(\mathrm{Fe} 5270+\mathrm{Fe} 5335) / 2$.
} 
panels). In terms of metallicity, however, the results were already very well constrained from the index analysis alone, so that the addition of photometry improves the results only marginally.

In Fig. 4.2, age and metallicity determinations are plotted against values taken from the literature for analyses of the poor SED set UBV (top panels), the rich set of 5 Lick indices as described above (middle panels), and the combined analysis using PRODUCT (bottom panels).

Two points are of particular interest: First, if only UBV are analysed, in terms of age and in terms of metallicity the results are severely uncertain, for old clusters and for clusters of intermediate age. In fact, the age metallicity degeneracy inherent in data which cover a wavelength baseline fromU through $\mathrm{V}$ only is so severe that the confidence intervals of our best solutions cover the full parameter space. Second, as a consequence, the results obtained from Lick analysis remain practically unchanged if both kinds of data are analysed simultaneously (cf. middle and bottom panels).

In Fig. 4.3, we compare our results for the analysis of the rich photometric data set (UBVJHK) and the poor spectral data set (using $\mathrm{H} \beta$ and $[\mathrm{MgFe}]$ only). In this case, the results from the separate analyses of the data sets are both comparable in their degrees of degeneracies, and the analysis of the two indices results in only slightly better constraints in both ages and metallicities than the analysis of the photometric data alone (top and middle panels).

In this case of a combination of a rich data set with a long wavelength baseline for the SEDs with a poor one with only two Lick indices, however, a combined analysis of both kinds of data using PRODUCT is most useful, resulting in age and metallicity determinations which are almost comparable in their precision with those of the combined analysis of the two rich data set presented in Fig. 4.1.

If the poor index data set is combined with the poor photometric one (Fig. 4.4 ), the results from the analysis of the two indices alone can slightly be improved in terms of metallicity determinations if analysed in combination with the rich spectral data set. However, in terms of ages best solutions and confidence intervals cannot be improved this way.

We conclude that the combined analysis of different data sets using PRODUCT is most useful if the constraints given by each single data set alone (Lick indices or colours) are of comparable accuracy. Since the age metallicity degeneracy is much more severe in colours than in indices, this means that age and metallicity determinations obtained by analysing Lick indices can substantially be improved only if a set of colours covering a wavelength basis as long as possible is available. On the other hand, if photometry of sufficient quality is available, the inclusion of only very few Lick indices into the analysis can severely improve the results.

\subsubsection{NGC 5128}

As a further example, we show results from our analysis of the globular cluster system of the nearest large elliptical galaxy, NGC 5128. Since much evidence 


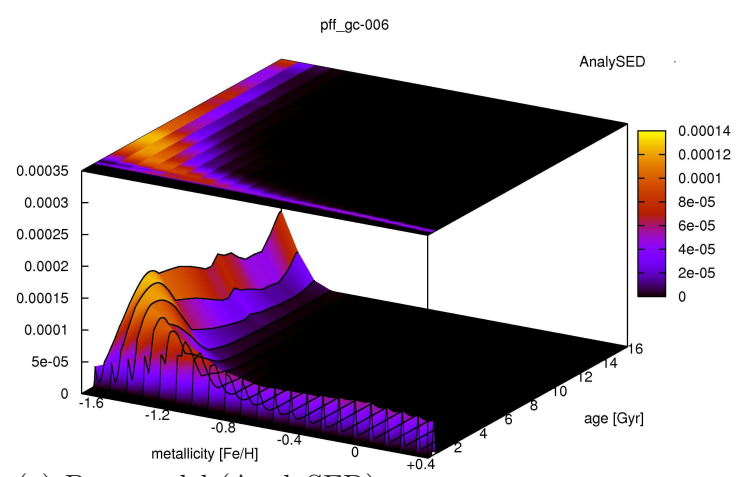

(a) Best model (AnalySED):

age $=5.55_{-4.32}^{+10.45} \mathrm{Gyr},[\mathrm{Fe} / \mathrm{H}]=-1.7_{-0.0}^{+0.7}$

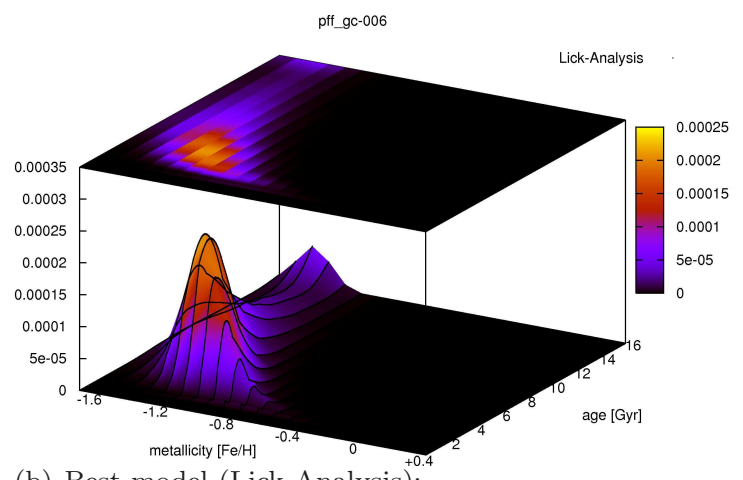

(b) Best model (Lick-Analysis):

age $=3.91_{-1.84}^{+12.09} \mathrm{Gyr},[\mathrm{Fe} / \mathrm{H}]=-1.2_{-0.3}^{+0.3}$

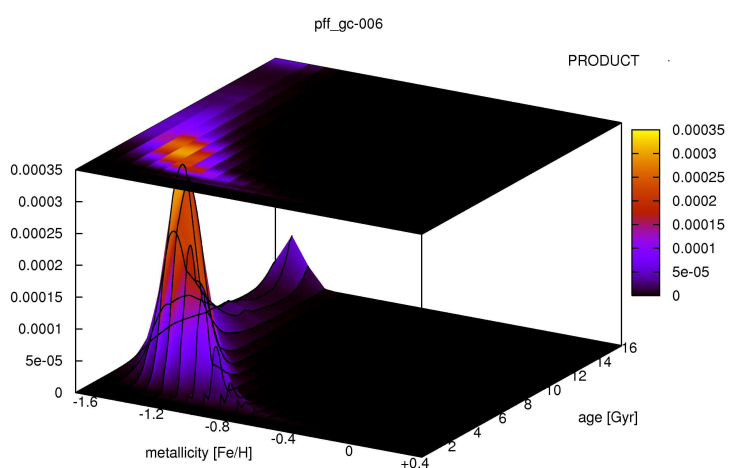

(c) Best model (PRODUCT):

age $=4.69_{-2.75}^{+3.12} \mathrm{Gyr},[\mathrm{Fe} / \mathrm{H}]=-1.4_{-0.2}^{+0.4}$

Fig. 4.6: Best models for NGC 5128 globular cluster pff_gc-006 using three different methods of analysis (data: Peng et al. 2004). The plots show the normalized probability space resulting from an analysis of the broad-band magnitudes UBVRI (a), the Lick indices $\mathrm{H} \beta, \mathrm{Mg} b, \mathrm{Mg}_{2}$, Fe5270, Fe5335 (b), and of a combination of both methods using PRODUCT (c). 

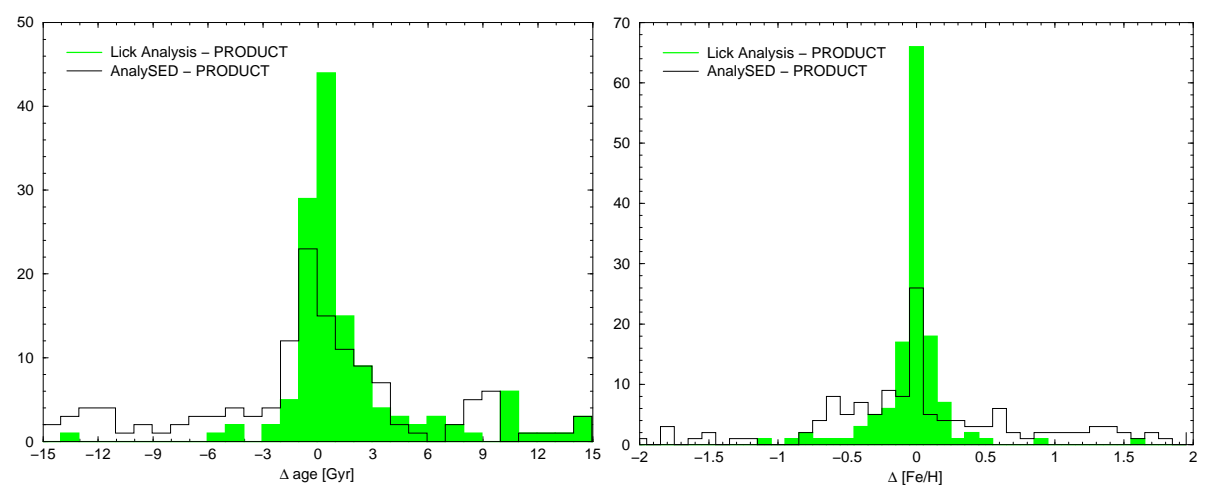

Fig. 4.7: Left: Histogram of differences between age determinations using AnalySED and Lick Analysis, respectively, and using PRODUCT for 135 GCs in NGC 5128. Right: Same as left, but for metallicities. 
exists that NGC 5128 has experienced one or more major merging events relatively recently, this galaxy is a very interesting object and, due to its proximity, suitable for very detailed investigations (for a complete review, see, e.g., Israel 1998).

For our analysis of the globular cluster system (GCS) of this giant elliptical, we took UBVRI photometry from Peng et al. (2004) and Lick indices $\mathrm{H} \beta$, $\mathrm{Mg} b, \mathrm{Mg}_{2}, \mathrm{Fe} 5270$, and Fe5335 from Peng (2005) for a very large sample of 135 clusters. The colours are dereddened following the extinction law given by Cardelli et al. (1989), using reddening values $\mathrm{E}(\mathrm{B}-\mathrm{V})$ for each cluster derived from Schlegel et al.(1998) as listed in Peng et al. (2004).

Since the spectral observations were originally intended to measure radial velocities only, the Lick indices measured on these spectra are of relatively poor quality, with typical errors between 0.5 and $1.0 \AA$. Thus, ages and metallicities determined from these data only are, especially in terms of ages, highly uncertain. On the other hand, due to the lack of NIR observations, results are highly uncertain as well as degenerate in terms of age and metallicity when analysing the UBVRI photometric data alone.

However, since Lick indices, analysed with the Lick Analysis Tool, give very reliable metallicity determinations even in cases where the ages are, in terms of $1 \sigma$ confidence intervals, very poorly constrained (cf. Sect. 4.4.1 and Lilly \& Fritze-v. Alvensleben 2006), a combined analysis of both data sets using PRODUCT can successfully break the age-metallicity degeneracy.

As an example, in Fig. 4.6 we show our results for cluster pff_gc-006 in terms of the resulting probability spaces for analyses of optical SEDs only (a), the 5 Lick indices only (b), and a combined analysis of both sets (c).

Both AnalySED and Lick analysis give comparable best ages between 4 and 6 Gyr, but the confidence intervals are extremely large, reaching more than 10 Gyr in both cases. As can be seen in this Fig., in case of SEDs this behaviour is due to a general degeneracy inherent in these optical colours which gives a very broad distribution of relatively high probabilities from young to old. In case of indices the very large confidence interval is due to a small but sufficiently well pronounced "second solution island" at high age.

However, if the photometric and spectral data are analysed simultaneously using PRODUCT he "intermediate age solution island" is much stronger, though the "high age solution" is still visible. Hence, the analysis with PRODUCT selects an intermediate age solution.

We will report in detail on our results on the GCS of NGC 5128 and their implications for the evolutionary history of this interesting galaxy a forthcoming paper. Within the scope of the paper, we here restrict ourselves on the description of the effect of PRODUCT on the results from the individual analyses of the photometric and spectral data sets, respectively, as shown above on the example of a single GC.

For this purpose, in Fig. 4.7 we show the differences between age (left panel) and metallicity (right panel) determinations, respectively, using AnalySED and PRODUCT (black histograms), and using Lick Analysis and PRODUCT (green shaded histograms) for the complete sample of 138 clusters: 


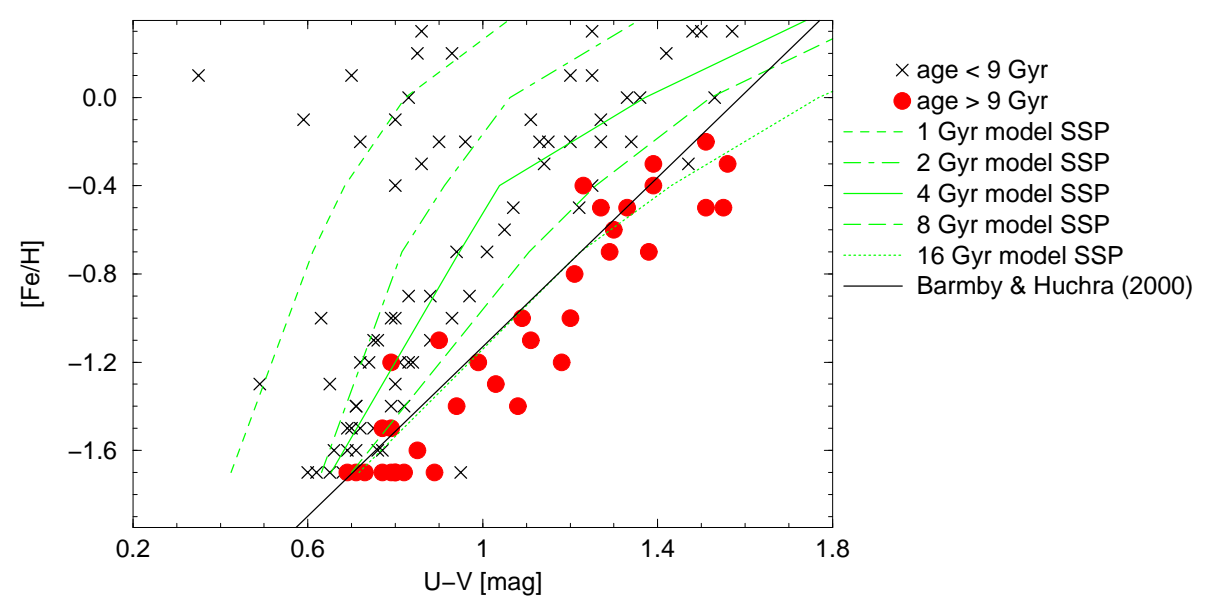

Fig. 4.8: Metallicities of star clusters in NGC 5128, determined by PRODUCT, vs U-V. Different symbols are used for two age bins. Also shown are GALEV model SSPs for 5 different ages and a colour metallicity relation taken from Barmby et al. (2000).

For example, $\Delta$ age $>0$ in the left panel of Fig. 4.7 means that, in case of the green shaded histogram, ages determined by PRODUCT are smaller than ages determined by Lick analysis alone. In this case, ages determined by Lick analysis are "corrected" downwards by the inclusion of the photometric data in the analysis process. Negative values of $\Delta$ age mean that ages from Lick index analysis alone are corrected upwards by PRODUCT with inclusion of broad band SEDs. Comparison of the open and shaded histograms shows that GC ages tend to become lower when all information is included than Lick-only determined ages and higher than SED-determined ages.

As we already showed in Lilly \& Fritze-v. Alvensleben (2006) in our comparison of Lick-based metallicities with independent CMD metallicities of Milky Way GCS, results from Lick index analysis are very accurate in terms of metallicity. The right panel of Fig. 4.7 shows that the inclusion of broad-band SEDs into the analysis process further improves these results in some cases.

\subsection{Intermediate-age GC populations and colour- metallicity-relations}

Quite common in the literature is the use of empirical color-metallicity-relations (CMRs) as given e.g. by Barmby et al. (2000) for the determination of GC metallicities from observed colors. All these relations available in the literature have been obtained for Galactic or M31 GCs, both of which are inherently old GCs.

Schulz et al. (2002) already showed, using GALEV evolutionary synthesis models, that there is good agreement between model and empirical colormetallicity relations for old ages and the metallicity range covered by Milky Way and M31 GCs. They also pointed out that for metallicities above those of 
Milky Way and M31 GCs, i.e. at $[\mathrm{Fe} / \mathrm{H}]$ i -0.5 , the relation steepens considerably so that metallicities derived from colors by extrapolation of the empirical relations to redder colors get severely overestimated. They also showed that the color-metallicity relation is significantly age dependent (see also Kurth et al. 1999), with appreciable deviations already visible between ages of $\gtrsim 12 \mathrm{Gyr}$ and $\sim 9$ Gyr.

Despite of this fact, CMRs are very often applied to the analysis of GCSs for which it is not a priori clear that all clusters are indeed homogeneously old. Part of the problem is, of course, that very often data are not available which would give hints to younger ages of some of the clusters; as we showed in this paper, the age metallicity degeneracy is very hard to break if only broad-band colours are available, especially if the wavelength basis does not extend from $\mathrm{U}$ through $\mathrm{K}$. However, even in cases where age differences are known, or expected, empirical CMRs are used. In case of the merger remnant NGC 5128, for example, CMRs were used e.q. by Rejkuba (2001) and Harris et al. (2004) to derive GC metallicities.

In Fig. 4.8, we plot our metallicity determinations vs. U-V for our sample of GC in NGC 5128 obtained by a combined analysis of our spectral and photometric data sets as presented in Sect. 4.4.2. Using our age determinations, we have coded our data points for two different age bins, clusters older than 9 Gyr (red circles), and clusters younger than 9 Gyr (mostly of intermediate age; black crosses). In the same plot, we added GALEV models for different ages as well as the CMR given by Barmby et al. (2000).

The plot shows a very good agreement between our 16 Gyr model, Barmby et al.'s CMR, and the metallicities determined for the set of old clusters. The clusters found to be of intermediate age by our analysis lie very well within the range of our models for theses ages, confirming the consistency of our age determinations. However, as can easily be read off the plot, metallicity determinations using a CMR calibrated on old clusters lead to seriously wrong results. For all GCs younger than 9 Gyr metallicities obtained via the empirical colormetallicity relation could be significantly underestimated - the more the younger the GC is.

We conclude that, if there is any doubt whether a given GCS consists of two or more subpopulations that might differ in age, CMRs can lead to serious metallicity misdeterminations and should not be used. A full SED or Lick or combined analysis is required in this cases to disentangle ages and metallicities.

\subsection{Summary/Conclusions}

I have developed a new method for the determination of ages and metallicities of individual star clusters by combining the information inherent in broad-band colour and Lick index measurements (PRODUCT). Basis

I have tested my new method using photometry and Lick indices for star clusters in the LMC, and in the elliptical galaxy NGC 5128, and find that age and metallicity determinations obtained by analysing Lick indices can substantially be improved if a set of colours covering a wavelength basis as long as possible, at best from $\mathrm{U}$ through $\mathrm{K}$, is available, even if only very few spectral 
indices are available.

I further point at the important fact that colour-metallicity relations widely used in the literature for determining star clusters metallicities are valid only for old GCs, and can by no means be applied to GCSs which contain subpopulations of intermediate age clusters.

\subsection{References}

Anders P. and Fritze-v. Alvensleben U., 2003, A\&A 401, 1063

Anders P., Bissantz N., Fritze-v. Alvensleben U., and de Grijs R., 2004, MNRAS 347,196

Barmby P., Huchra J.P., Brodie J.P., Forbes D.A., Schroder L.L., and Grillmair C.J., 2000, AJ 119, 727

Beasley M.A., Hoyle F., and Sharples R.M., 2002, MNRAS 336, 168

Bertelli G., Bressan A., Chiosi C., Fagotto F., and Nasi E., 1994, A\&AS 106, 275

Bica E., Claria J.J., Dottori H., Santos J.F.C. Jr., and Piatti A.E., 1996, ApJS 102,57

Bicker J., Fritze-v. Alvensleben U., Möller C.S., and Fricke K.J., 2004, A\&A 413,37

Cardelli J.A., Clayton G.C., and Mathis J.S., 1989, ApJ 345, 245

Chabrier G. and Baraffe I., 1997, A\&A 327, 1039

Dirsch B., Richtler T., Gieren W.P., and Hilker M., 2000, A\&A 360, 133

Dutra C.M., Bica E., Claria J.J., and Piatti A.E., 1999, MNRAS 305, 373

Elson R.A. and Fall S.M., 1988, AJ 96, 1383

Geisler D., Bica E., Dottori H., Claria J.J., Piatti A.E., and Santos J.F.C. Jr., 1997, AJ 114, 1920

Girardi L., Bressan A., Chiosi C., Bertelli G., and Nasi E., 1996, A\&AS 117, 113

Gordon K.D., Clayton G.C., Misselt K.A., Landolt A.U., and Wolff M.J., 2003, ApJ 594, 279

Harris G.L.H., Harris W.E., Geisler D., 2004, AJ 128, 723

Israel F.P., 1998, A\&A Rev. 8, 237

Lejeune T., Cuisinier F., and Buser R., 1997, A\&AS 125, 229

Lejeune T., Cuisinier F., and Buser R., 1998, A\&AS 130, 65

Lilly T. and Fritze-v. Alvensleben U., 2006, A\&A 457, 467

Mackey A.D. and Gilmore G.F., 2003, MNRAS 338, 85

Mould J. and Aaronson M., 1982, ApJ 263, 629

Olsen K.A.G., Hodge P.W., Mateo M., Olszewski E.W., Schommer R.A., Suntzeff N.B., and Walker A.R., 1998, MNRAS 300, 665

Olszewski E.W., Schommer R.A., Suntzeff N.B., and Harris H.C., 1991, AJ 101, 515 
Peng E.W., Ford H.C., and Freeman K.C., 2004, ApJS 150, 367

Peng E.W., 2005, private comunication

Pessev P.M., Goudfrooij P., Puzia T.H., and Chandar R., 2006, AJ 132, 781

Rabin D., 1982, ApJ 261, 85

Rejkuba M., 2001, A\&A 369, 812

Salpeter E.E., 1955, ApJ 121, 161

Schlegel D.J., Finkbeiner D.P., and Davis M., 1998, ApJ 500, 525

Schulz J., Fritze-v. Alvensleben U., Möller C.S., and Fricke K.J., 2002, A\&A 392,1

Thomas D., Maraston C. and Bender R., 2003, MNRAS 339, 897

Worthey G., Faber S.M., González J.J., and Burstein D., 1994, ApJS 94, 687

Worthey G. and Ottaviani D.L., 1997, ApJS 111, 377

\subsection{Appendix: Results for ages and metallicities of LMC star clusters}

In this appendix, we give all results from our analysis of different sets of SEDs and indices for $11 \mathrm{LMC}$ star clusters, together with ages and metallicities to compare with from the literature, as described in Sect. 4.4.1. 
Table 4.1: Ages and metallicities for 11 LMC star clusters analysed using broad-band photometry UBVJHKs for AnalySED, the 5 Lick indices $\mathrm{H} \beta, \mathrm{Mg} b, \mathrm{Mg}_{2}$, Fe5270, Fe5335 for the Lick analysis, and PRODUCT using both sets of data.

\begin{tabular}{lccccccccc}
\hline \hline & \multicolumn{2}{c}{ AnalySED (UBVJHKs) } & \multicolumn{2}{c}{ Lick-Analysis $(5$ indices) } & \multicolumn{2}{c}{ PRODUCT } & \multicolumn{3}{c}{ Literature } \\
& Age $(\mathrm{Gyr})$ & {$[\mathrm{Fe} / \mathrm{H}]$} & Age $(\mathrm{Gyr})$ & {$[\mathrm{Fe} / \mathrm{H}]$} & Age $(\mathrm{Gyr})$ & {$[\mathrm{Fe} / \mathrm{H}]$} & Age $(\mathrm{Gyr})$ & {$[\mathrm{Fe} / \mathrm{H}]$} & Sources \\
\hline NGC1718 & $0.32_{-0.06}^{+0.31}$ & $0.4_{-0.1}^{+0.0}$ & $2.75_{-0.73}^{+1.80}$ & $-1.1_{-0.1}^{+0.1}$ & $2.00_{-0.22}^{+1.34}$ & $-0.9_{-0.2}^{+0.1}$ & $1.78_{-0.90}^{+1.80}$ & -0.42 \\
NGC1751 & $0.12_{-0.02}^{+0.03}$ & $0.0_{-0.3}^{+0.2}$ & $1.14_{-0.20}^{+0.42}$ & $-0.3_{-0.2}^{+0.2}$ & $0.84_{-0.10}^{+0.11}$ & $-0.1_{-0.1}^{+0.1}$ & $1.40_{-0.90}^{+2.10}$ & $-0.18 \pm 0.20$ & $2 / 9$ \\
NGC1786 & $10.50_{-6.89}^{+5.50}$ & $-1.6_{-0.1}^{+0.2}$ & $15.14_{-3.74}^{+0.86}$ & $-1.6_{-0.0}^{+0.0}$ & $15.26_{-3.89}^{+0.74}$ & $-1.6_{-0.0}^{+0.0}$ & $15.10_{-3.10}^{+3.10}$ & $-1.87 \pm 0.20$ & $2 / 7$ \\
NGC1806 & $0.86_{-0.15}^{+6.94}$ & $0.4_{-1.2}^{+0.0}$ & $2.32_{-0.44}^{+0.58}$ & $-0.7_{-0.1}^{+0.1}$ & $2.44_{-0.53}^{+0.68}$ & $-0.7_{-0.1}^{+0.1}$ & $0.50_{-0.10}^{+0.13}$ & $-0.71 \pm 0.24$ & $3 / 3$ \\
NGC1846 & $0.77_{-0.17}^{+4.92}$ & $0.4_{-1.1}^{+0.0}$ & $1.90_{-0.60}^{+0.32}$ & $-0.7_{-0.1}^{+0.1}$ & $1.66_{-0.11}^{+0.48}$ & $-0.6_{-0.1}^{+0.0}$ & $2.20_{-0.80}^{+1.30}$ & $-0.70 \pm 0.20$ & $2 / 9$ \\
NGC1856 & $0.12_{-0.02}^{+0.00}$ & $-1.7_{-0.0}^{+1.4}$ & $0.60_{-0.07}^{+0.08}$ & $-0.2_{-0.1}^{+0.1}$ & $0.50_{-0.00}^{+0.04}$ & $-0.3_{-0.0}^{+0.1}$ & $0.12_{-0.06}^{+0.12}$ & -0.52 & $1 / 8$ \\
NGC1898 & $3.27_{-2.27}^{+2.77}$ & $-1.7_{-0.0}^{+0.6}$ & $16.00_{-9.51}^{+0.00}$ & $-1.3_{-0.0}^{+0.2}$ & $16.00_{-4.06}^{+0.00}$ & $-1.4_{-0.0}^{+0.1}$ & $14.00_{-2.30}^{+2.30}$ & $-1.37 \pm 0.20$ & $2 / 6$ \\
NGC1916 & $14.70_{-12.39}^{+1.30}$ & $-1.6_{-0.1}^{+0.3}$ & $6.17_{-1.58}^{+9.83}$ & $-1.6_{-0.1}^{+0.1}$ & $6.36_{-1.37}^{+2.05}$ & $-1.6_{-0.0}^{+0.1}$ & $15.85_{--2.97}^{+3.65}$ & $-2.08 \pm 0.20$ & $2 / 1$ \\
NGC1978 & $1.05_{-0.55}^{+4.95}$ & $-1.0_{-0.7}^{+1.0}$ & $1.91_{-0.34}^{+0.45}$ & $-0.4_{-0.1}^{+0.1}$ & $1.46_{-0.10}^{+0.58}$ & $-0.4_{-0.1}^{+0.0}$ & $2.50_{-1.25}^{+2.50}$ & $-0.424 \pm 0.20$ & $2 / 8$ \\
NGC1987 & $0.48_{-0.19}^{+0.37}$ & $0.4_{-0.4}^{+0.0}$ & $1.64_{-0.25}^{+0.33}$ & $-0.6_{-0.1}^{+0.1}$ & $1.59_{-0.51}^{+0.20}$ & $-0.6_{-0.0}^{+0.3}$ & $1.82_{-1.00}^{+2.20}$ & $-0.50 \pm 0.20$ & $5 / 9$ \\
NGC2019 & $5.01_{-3.03}^{+10.99}$ & $-1.7_{-0.0}^{+0.1}$ & $6.89_{-1.10}^{+9.11}$ & $-1.4_{-0.1}^{+0.0}$ & $15.91_{-9.48}^{+0.09}$ & $-1.6_{-0.0}^{+0.1}$ & $17.80_{-3.20}^{+3.20}$ & $-1.81 \pm 0.20$ & $2 / 6$ \\
\hline
\end{tabular}

a Sources for literature values (metallicity/age): 1 Mackey \& Gilmore (2003); 2 Olszewski et al. (1991); 3 Dirsch et al. (2000); 4 Rabin (1982); 5 Elson \& Fall (1988); 6 Mould \& Aaronson (1982). 7 Geisler et al. (1997); 8 Olsen et al. (1998). 
Table 4.2: Same as Fig. 4.1, but using UBV only for AnalySED.

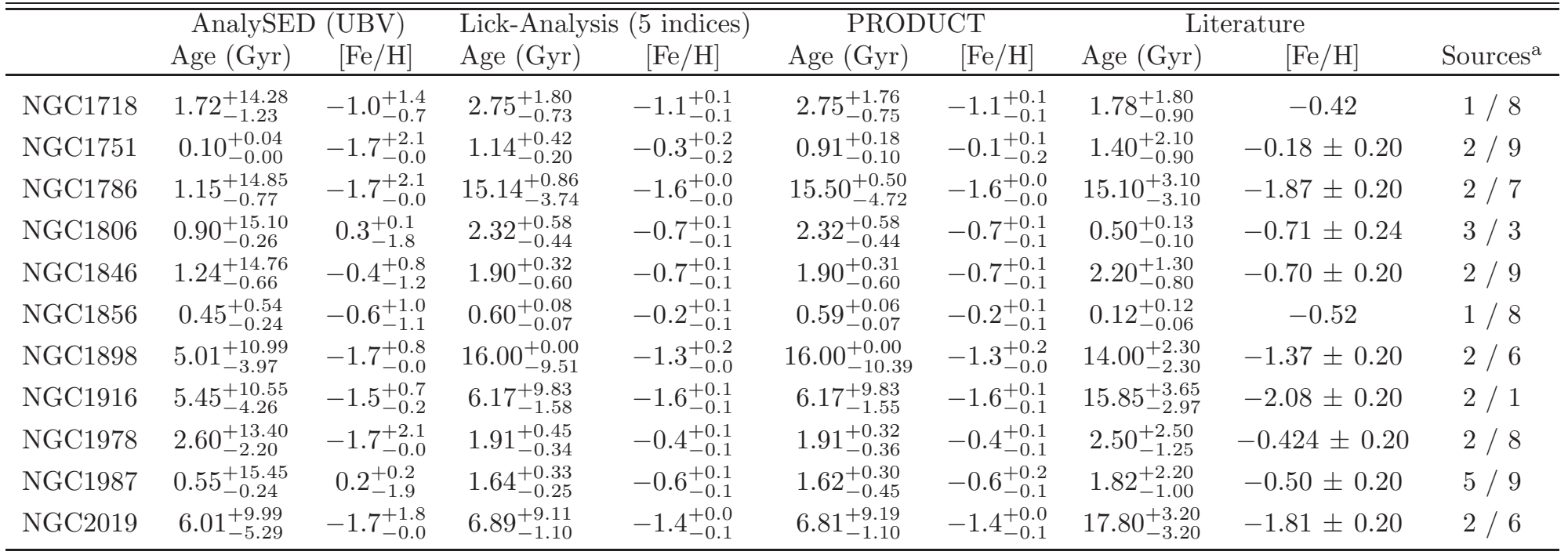

a Sources for literature values (metallicity/age): 1 Mackey \& Gilmore (2003); 2 Olszewski et al. (1991); 3 Dirsch et al. (2000); 4 Rabin (1982); 5 Elson \& Fall (1988); 6 Mould \& Aaronson (1982). 7 Geisler et al. (1997); 8 Olsen et al. (1998). 
Table 4.3: Same as Fig. 4.1, but using Lick indices $\mathrm{H} \beta$ \& $[\mathrm{MgFe}]$ only for the Lick analysis.

\begin{tabular}{cccccccccc}
\hline \hline & \multicolumn{2}{c}{ AnalySED (UBVJHKs) } & \multicolumn{2}{c}{ Lick-Analysis $(\mathrm{H} \beta,[\mathrm{MgF}])$} & \multicolumn{2}{c}{ PRODUCT } & \multicolumn{3}{c}{ Literature } \\
& Age $(\mathrm{Gyr})$ & {$[\mathrm{Fe} / \mathrm{H}]$} & Age $(\mathrm{Gyr})$ & {$[\mathrm{Fe} / \mathrm{H}]$} & Age $(\mathrm{Gyr})$ & {$[\mathrm{Fe} / \mathrm{H}]$} & Age $(\mathrm{Gyr})$ & {$[\mathrm{Fe} / \mathrm{H}]$} \\
\hline NGC1718 & $0.32_{-0.06}^{+0.31}$ & $0.4_{-0.1}^{+0.0}$ & $3.01_{-2.91}^{+2.04}$ & $-1.2_{-0.1}^{+0.4}$ & $2.26_{-0.52}^{+1.04}$ & $-1.0_{-0.2}^{+0.2}$ & $1.78_{-0.90}^{+1.80}$ & -0.42 \\
NGC1751 & $0.12_{-0.02}^{+0.03}$ & $0.0_{-0.3}^{+0.2}$ & $1.13_{-1.03}^{+0.51}$ & $-0.3_{-1.4}^{+0.2}$ & $0.11_{-0.00}^{+0.83}$ & $-0.8_{-0.1}^{+0.8}$ & $1.40_{-0.90}^{+2.10}$ & $-0.18 \pm 0.20$ & $2 / 9$ \\
NGC1786 & $10.50_{-6.89}^{+5.50}$ & $-1.6_{-0.1}^{+0.2}$ & $13.80_{-3.42}^{+1.38}$ & $-1.6_{-0.0}^{+0.0}$ & $13.18_{-2.56}^{+1.65}$ & $-1.6_{-0.0}^{+0.0}$ & $15.10_{-3.10}^{+3.10}$ & $-1.87 \pm 0.20$ & $2 / 7$ \\
NGC1806 & $0.86_{-0.15}^{+6.94}$ & $0.4_{-1.2}^{+0.0}$ & $0.11_{-0.01}^{+3.23}$ & $-0.8_{-0.4}^{+0.1}$ & $2.38_{-0.49}^{+0.85}$ & $-0.7_{-0.2}^{+0.1}$ & $0.50_{-0.10}^{+0.13}$ & $-0.71 \pm 0.24$ & $3 / 3$ \\
NGC1846 & $0.77_{-0.17}^{+4.92}$ & $0.4_{-1.1}^{+0.0}$ & $1.88_{-1.78}^{+0.38}$ & $-0.7_{-1.0}^{+0.1}$ & $1.70_{-0.16}^{+0.45}$ & $-0.6_{-0.1}^{+0.0}$ & $2.20_{-0.80}^{+1.30}$ & $-0.70 \pm 0.20$ & $2 / 9$ \\
NGC1856 & $0.12_{-0.02}^{+0.00}$ & $-1.7_{-0.0}^{+1.4}$ & $0.54_{-0.12}^{+0.05}$ & $0.0_{-0.1}^{+0.2}$ & $0.50_{-0.00}^{+0.02}$ & $-0.4_{-0.0}^{+0.1}$ & $0.12_{-0.06}^{+0.12}$ & -0.52 & $1 / 8$ \\
NGC1898 & $3.27_{-2.27}^{+2.77}$ & $-1.7_{-0.0}^{+0.6}$ & $9.14_{-3.38}^{+6.86}$ & $-1.1_{-0.1}^{+0.1}$ & $15.88_{-6.81}^{+0.12}$ & $-1.4_{-0.1}^{+0.0}$ & $14.00_{-2.30}^{+2.30}$ & $-1.37 \pm 0.20$ & $2 / 6$ \\
NGC1916 & $14.70_{-12.39}^{+1.30}$ & $-1.6_{-0.1}^{+0.3}$ & $6.10_{-0.96}^{+9.90}$ & $-1.6_{-0.1}^{+0.0}$ & $6.31_{-1.68}^{+2.78}$ & $-1.6_{-0.1}^{+0.1}$ & $15.85_{-2.97}^{+3.65}$ & $-2.08 \pm 0.20$ & $2 / 1$ \\
NGC1978 & $1.05_{-0.55}^{+4.95}$ & $-1.0_{-0.7}^{+1.0}$ & $0.10_{-0.00}^{+2.53}$ & $-0.8_{-0.3}^{+0.4}$ & $1.26_{-0.03}^{+0.15}$ & $-0.4_{-0.1}^{+0.0}$ & $2.50_{-1.25}^{+2.50}$ & $-0.424 \pm 0.20$ & $2 / 8$ \\
NGC1987 & $0.48_{-0.19}^{+0.37}$ & $0.4_{-0.4}^{+0.0}$ & $0.10_{-0.00}^{+1.80}$ & $-1.6_{-0.1}^{+1.1}$ & $1.53_{-0.38}^{+0.33}$ & $-0.7_{-0.1}^{+0.2}$ & $1.82_{-1.00}^{+2.20}$ & $-0.50 \pm 0.20$ & $5 / 9$ \\
NGC2019 & $5.01_{-3.03}^{+10.99}$ & $-1.7_{-0.0}^{+0.1}$ & $8.26_{-2.52}^{+7.74}$ & $-1.5_{-0.1}^{+0.1}$ & $15.88_{-8.93}^{+0.12}$ & $-1.6_{-0.1}^{+0.0}$ & $17.80_{-3.20}^{+3.20}$ & $-1.81 \pm 0.20$ & $2 / 6$ \\
\hline
\end{tabular}

a Sources for literature values (metallicity/age): 1 Mackey \& Gilmore (2003); 2 Olszewski et al. (1991); 3 Dirsch et al. (2000); 4 Rabin (1982); 5 Elson \& Fall (1988); 6 Mould \& Aaronson (1982). 7 Geisler et al. (1997); 8 Olsen et al. (1998). 
Table 4.4: Same as Fig. 4.1, but using UBV only for AnalySED, and H $\beta$ \& [MgFe] only for the Lick analysis.

\begin{tabular}{cccccccccc}
\hline \hline & \multicolumn{2}{c}{ AnalySED (UBV) } & \multicolumn{2}{c}{ Lick-Analysis $(\mathrm{H} \beta,[\mathrm{MgF}])$} & \multicolumn{2}{c}{ PRODUCT } & \multicolumn{3}{c}{ Literature } \\
& Age $(\mathrm{Gyr})$ & {$[\mathrm{Fe} / \mathrm{H}]$} & Age $(\mathrm{Gyr})$ & {$[\mathrm{Fe} / \mathrm{H}]$} & Age $(\mathrm{Gyr})$ & {$[\mathrm{Fe} / \mathrm{H}]$} & Age $(\mathrm{Gyr})$ & {$[\mathrm{Fe} / \mathrm{H}]$} \\
\hline NGC1718 & $1.72_{-1.23}^{+14.28}$ & $-1.0_{-0.7}^{+1.4}$ & $3.01_{-2.91}^{+2.04}$ & $-1.2_{-0.1}^{+0.4}$ & $2.98_{-0.91}^{+2.02}$ & $-1.2_{-0.1}^{+0.2}$ & $1.78_{-0.90}^{+1.80}$ & -0.42 \\
NGC1751 & $0.10_{-0.00}^{+0.04}$ & $-1.7_{-0.0}^{+2.1}$ & $1.13_{-1.03}^{+0.51}$ & $-0.3_{-1.4}^{+0.2}$ & $0.10_{-0.00}^{+0.01}$ & $-1.7_{-0.0}^{+0.3}$ & $1.40_{-0.90}^{+2.10}$ & $-0.18 \pm 0.20$ & $2 / 8$ \\
NGC1786 & $1.15_{-0.77}^{+14.85}$ & $-1.7_{-0.0}^{+2.1}$ & $13.80_{-3.42}^{+1.38}$ & $-1.6_{-0.0}^{+0.0}$ & $12.59_{-3.04}^{+2.65}$ & $-1.6_{-0.1}^{+0.0}$ & $15.10_{-3.10}^{+3.10}$ & $-1.87 \pm 0.20$ & $2 / 7$ \\
NGC1806 & $0.90_{-0.26}^{+15.10}$ & $0.3_{-1.8}^{+0.1}$ & $0.11_{-0.01}^{+3.23}$ & $-0.8_{-0.4}^{+0.1}$ & $2.44_{-0.46}^{+0.85}$ & $-0.8_{-0.2}^{+0.1}$ & $0.50_{-0.10}^{+0.13}$ & $-0.71 \pm 0.24$ & $3 / 3$ \\
NGC1846 & $1.24_{-0.66}^{+14.76}$ & $-0.4_{-1.2}^{+0.8}$ & $1.88_{-1.78}^{+0.38}$ & $-0.7_{-1.0}^{+0.1}$ & $1.88_{-0.64}^{+0.39}$ & $-0.7_{-0.1}^{+0.1}$ & $2.20_{-0.80}^{+1.30}$ & $-0.70 \pm 0.20$ & $2 / 9$ \\
NGC1856 & $0.45_{-0.24}^{+0.54}$ & $-0.6_{-1.1}^{+1.0}$ & $0.54_{-0.12}^{+0.05}$ & $0.0_{-0.1}^{+0.2}$ & $0.53_{-0.12}^{+0.06}$ & $0.0_{-0.1}^{+0.2}$ & $0.12_{-0.06}^{+0.12}$ & -0.52 & $1 / 8$ \\
NGC1898 & $5.01_{-3.97}^{+10.99}$ & $-1.7_{-0.0}^{+0.8}$ & $9.14_{-3.38}^{+6.86}$ & $-1.1_{-0.1}^{+0.1}$ & $10.00_{-5.03}^{+6.00}$ & $-1.2_{-0.1}^{+0.2}$ & $14.00_{-2.30}^{+2.30}$ & $-1.37 \pm 0.20$ & $2 / 6$ \\
NGC1916 & $5.45_{-4.26}^{+10.55}$ & $-1.5_{-0.2}^{+0.7}$ & $6.10_{-0.96}^{+9.90}$ & $-1.6_{-0.1}^{+0.0}$ & $6.10_{-0.98}^{+9.90}$ & $-1.6_{-0.1}^{+0.0}$ & $15.85_{-2.97}^{+3.65}$ & $-2.08 \pm 0.20$ & $2 / 1$ \\
NGC1978 & $2.60_{-2.20}^{+13.40}$ & $-1.7_{-0.0}^{+2.1}$ & $0.10_{-0.00}^{+2.53}$ & $-0.8_{-0.3}^{+0.4}$ & $2.00_{-0.44}^{+0.46}$ & $-0.5_{-0.1}^{+0.1}$ & $2.50_{-1.25}^{+2.50}$ & $-0.424 \pm 0.20$ & $2 / 8$ \\
NGC1987 & $0.55_{-0.24}^{+15.45}$ & $0.2_{-1.9}^{+0.2}$ & $0.10_{-0.00}^{+1.80}$ & $-1.66_{-0.1}^{+1.1}$ & $1.22_{-0.08}^{+0.66}$ & $-0.6_{-0.2}^{+0.1}$ & $1.82_{-1.00}^{+2.20}$ & $-0.50 \pm 0.20$ & $5 / 9$ \\
NGC2019 & $6.01_{-5.29}^{+9.99}$ & $-1.7_{-0.0}^{+1.8}$ & $8.26_{-2.52}^{+7.74}$ & $-1.5_{-0.1}^{+0.1}$ & $8.09_{-2.31}^{+7.91}$ & $-1.5_{-0.1}^{+0.1}$ & $17.80_{-3.20}^{+3.20}$ & $-1.81 \pm 0.20$ & $2 / 6$ \\
\hline
\end{tabular}

a Sources for literature values (metallicity/age): 1 Mackey \& Gilmore (2003); 2 Olszewski et al. (1991); 3 Dirsch et al. (2000); 4 Rabin (1982); 5 Elson \& Fall (1988); 6 Mould \& Aaronson (1982). 7 Geisler et al. (1997); 8 Olsen et al. (1998). 


\section{Chapter 5}

\section{Applications to the globular cluster system of NGC $5128^{1}$}

\subsection{Introduction}

The nearest large elliptical galaxy NGC 5128 is the only elliptical in a relatively small group of galaxies, M83. For this exceptionally interesting object, which also hosts a strong radio source ("Centaurus A"), much evidence exists that the galaxy has experienced one or more major merging events relatively recently (i.e., within the last few Gyrs), reflected, e.g., in a lot of fine structure like loops, shells, ripples and tails. Along with the radio source, a prominent disklike dust lane, seen on optical images around the inner part of the galaxy, is much discussed in the literature. Due to its proximity of only about $3.5 \mathrm{Mpc}$, reflected in a distance modulus (m-M) of only about $28 \mathrm{mag}$, NGC 5128 is an object suitable for very detailed investigations of nearly all aspects accessible through observations (for a complete review, see, e.g., Israel 1998).

The violent evolutionary history of NGC 5128 is expected to have left traces in the properties of its globular cluster system (GCS), which presumably consists of at least two or maybe more cluster subpopulations characterized by different ages and metallicities, originating partly from newly formed and/or captured clusters during recent interactions. Previous investigations of the GCS of NGC 5128, e.g. by Peng et al. (2004b), Harris et al. (2004), Rejkuba (2001) have found a bimodal optical colour distribution for the GCS of NGC 5128.

For NGC 5128 as well as for essentially all the more than 100 bimodal GCSs found so far in elliptical and S0 galaxies (e.g., see Gebhard \& Kissler-Patig 1999, Kundu \& Whitmore 2001a, b) the colour of the blue peak seems to be fairly universal and blue peak GCs are believed to be old and metal-poor. The colour of the red peak varies from galaxy to galaxy as does its height relative to that of the blue one. Ages and metallicities of the red peak GCs in E/S0s are still under debate.

\footnotetext{
${ }^{1}$ An extended version of this chapter is in preparation for submission to be published in A\&A.
} 

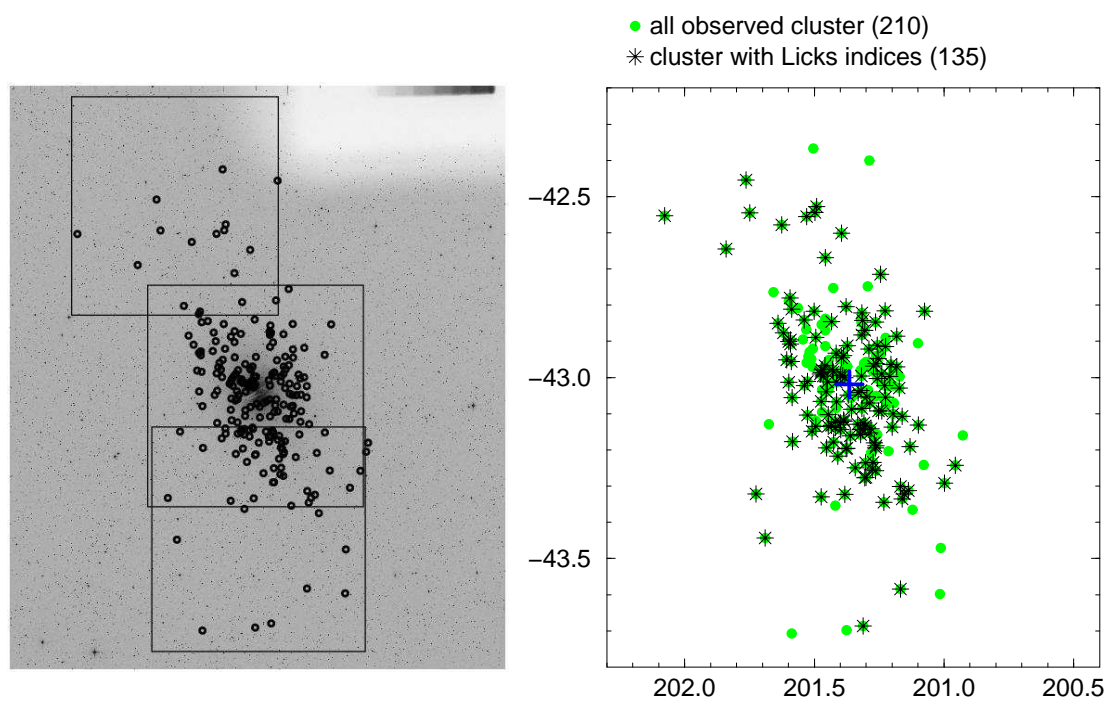

Fig. 5.1: Positions of all clusters for which UBVRI photometry is available, overplotted on a DSS image of NGC 5128 (left panel; taken from Peng et al. 2004). The right panel reproduces this figure, but also show the position of the subsample of clusters for which spectral indides are available as well.

In this chapter, I apply the analysis tools that I have developed in the framework of this thesis to the GCS of this object.

\subsection{Cluster sample and data analysis}

For my analysis of the cluster system of this object, I used UBVRI photometry obtained with the CTIO Mosaic camera at the Blanco $4 \mathrm{~m}$ telescope from Peng et al. (2004a), available for a large sample of 215 clusters, and Lick indices $\mathrm{H} \beta, \mathrm{Mg} b, \mathrm{Mg}_{2}, \mathrm{Fe} 5270$, and Fe5335 from Peng (2005) for a subsample of 135 clusters, measured on spectra obtained with AAO 2dF and CTIO Hydra.

The spectral observations were originally intended to measure radial velocities only (cf. Peng et al. 2004a). Therefore, the Lick indices measured on these spectra are of relatively poor quality, with typical errors between 0.5 and $1.0 \AA$ for all obtained indices.

In Fig. 5.1 I show the positions of all clusters for which UBVRI photometry is available, overplotted on a Digital Sky Survey image of NGC 5128 taken from Peng et al. (2004a) (left panel). The right panel reproduces this figure, but also shows the positions of the subsample of clusters for which spectral indices are available as well (in J2000; x-axis indicates R.A., y-axis Dec.). The center of the galaxy is marked by a blue plus-sign.

As can be seen in the left panel, no cluster have been observed within the dust belt near the center of NGC 5128; therefore, it is not expected that internal reddening is a very serious problem for the observed clusters. 

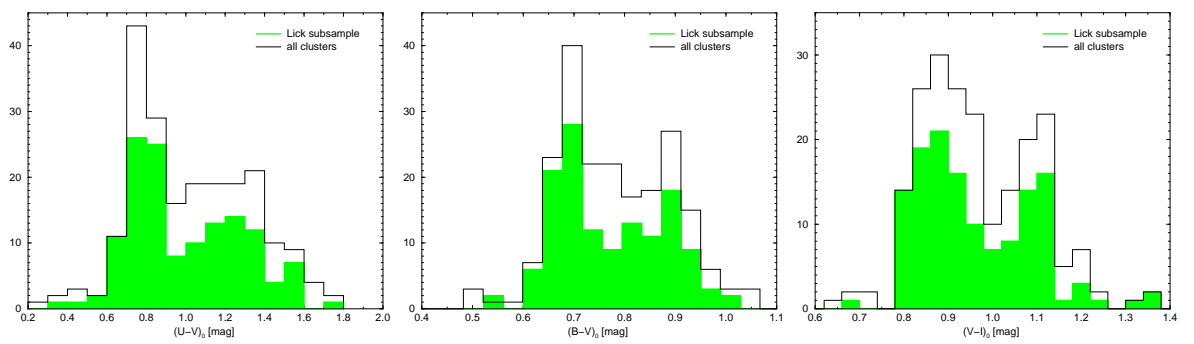

Fig. 5.2: Histograms of dereddened colours $\mathrm{U}-\mathrm{V}$ (left panel), B-V (middle panel), and V-I (right panel) for the complete sample of clusters (black histogram), and for the subsample of clusters with spectral observations (green shaded area).

The colours are dereddened following the extinction law given by Cardelli et al. (1989), using reddening values $\mathrm{E}(\mathrm{B}-\mathrm{V})$ for each cluster derived from Schlegel et al.(1998) as listed in Peng et al. (2004a).

In Fig. 5.2, I show the colour distribution of the dereddened clusters in $\mathrm{U}-$ $\mathrm{V}, \mathrm{B}-\mathrm{V}$, and $\mathrm{V}-\mathrm{I}$ for the complete sample of clusters with UBVRI photometry (black histogram) along with the subsample of clusters for which both photometry and spectral information in terms of Lick indices are available.

All colours show a very well constrained bimodality in their distributions. However, this does not necessarily mean that the GCS consists of only two well distinguished cluster subpopulations, e.g. an old metal-poor plus a younger metal-rich subpopulation, as it is often assumed for early types galaxies throughout the literature. Instead, as shown by Fritze-v. Alvensleben (2004), it is very well possible that several subpopulations "hide" in one colour peak. Taking Lick indices into consideration in addition to broad-band optical colours should allow to test for this.

Due to the quality of the Lick index measurements, ages and metallicities obtained from these data are fairly uncertain, especially in terms of age. Due to the lack of NIR observations, results are fairly uncertain as well both in terms of ages and metallicities when analysing the UBVRI photometric data. However, as has been shown in chapter 4 of this thesis, a combined analysis of both datasets can significantly improve the results.

Following the method introduced there, I have simultaneously analysed the whole dataset of both photometric data and spectral indices with the analysis tool PRODUCT developed in chapter 4. The results I get from this combined analysis are of acceptable quality, as shown by their $1 \sigma$ confidence levels, for the majority of GCs. For non-negligible subset of clusters, however, age determinations still have confidence intervals of up to $10 \mathrm{Gyr}$ or even more, unacceptable for interpretation.

For the purpose of this chapter, I have therefore restricted my interpretation to clusters for which the PRODUCT analysis gives $\pm 1 \sigma$ confidence intervals of less than $6 \mathrm{Gyr}$ in age, and less than $0.7 \mathrm{dex}$ in $[\mathrm{Fe} / \mathrm{H}]$. This reduces the sample to 80 clusters for which independently determined ages and metallicities are reliable. 


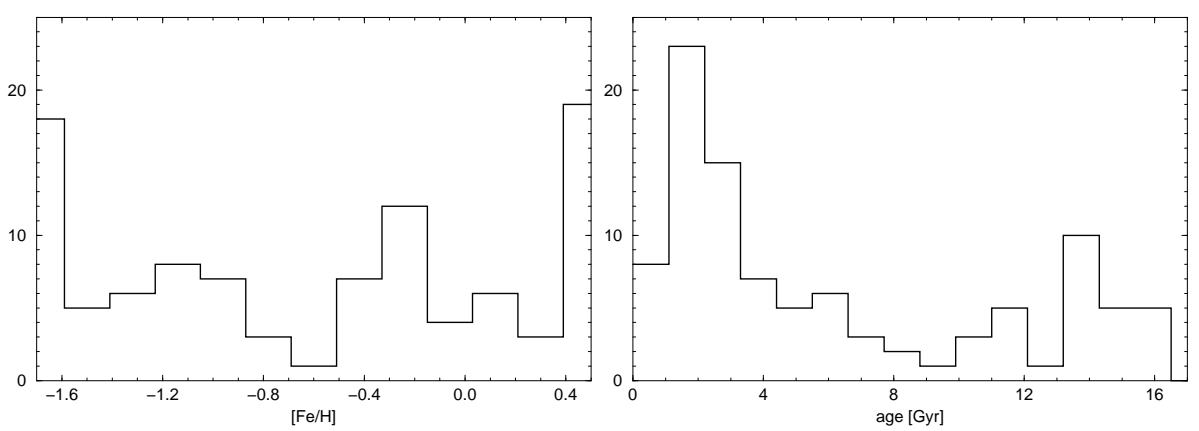

Fig. 5.3: Metallicity (left) and age (right) distribution of the NGC 5128 cluster sample.

Because the results are not as precise as one might has hoped, even for this reduced sample one should be cautious about results for individual clusters. The size of this sample with 80 clusters, however, is sufficiant to reliably discuss the properties of the GCS as a whole, in particular with respect to possible subsamples within the red or blue colour peaks.

\subsection{Results and discussion}

In this section, I present and discuss my results, and give some remarks about possible implications for the evolutionary history of NGC 5128.

\subsubsection{Age and metallicity distributions}

Fig. 5.3 show histograms of my results in term of metallicities (left panel) and ages (right panel). Bimodality in the distribution of both ages and metallicities is clearly seen, the metallicities being consistent with spectroscopy-based metallicity determinations by Held et al. (2002), which found a bimodal distribution with mean metallicities $[\mathrm{Fe} / \mathrm{H}]=-1.2$ and $[\mathrm{Fe} / \mathrm{H}]=-0.3$, respectively. The age distribution, on the other hand, is characterized by an unexpected high number of intermediate age clusters with ages between 1 and 3 Gyr.

However, some remarks about the metallicity distribution have to be made, concerning the peaks in both the lowest and the highest metallicity bin.

For the lowest bin, this is presumably an artefact due to the restricted parameter space of our analysis: The lowest metallicity included in the model grid is $[\mathrm{Fe} / \mathrm{H}]=-1.7$; therefore, clusters with lower metallicities are expected to accumulate at the "low-metallicity edge". The high-metallicity peak is more difficult to explain. Since significant numbers of clusters with metallicities higher than $[\mathrm{Fe} / \mathrm{H}]=0.4$ are not expected, this result presumably still suffers from some age-metallicity degeneracy. Since for all these clusters in the highest metallicity bin my analysis gives intermediate ages, it cannot be ruled out that a fraction of these clusters are, instead of being intermediate age and metal-rich, in fact older and less metal rich. 


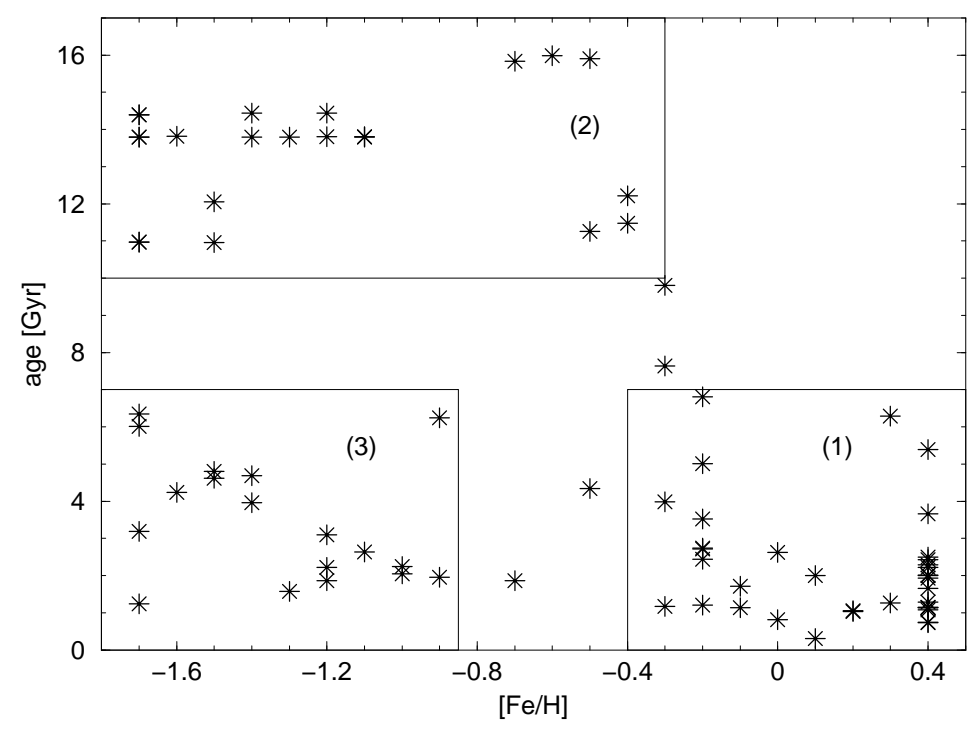

Fig. 5.4: Age vs metallicity for the NGC 5128 cluster sample. Three different subpopulations are emphasized by boxes.

In Fig. 5.4, age vs metallicity is plotted for all clusters. In this figure, I will distinguish between three populations:

1. A population of metal-rich clusters with young to intermediate ages (metallicities $-0.4 \leq[\mathrm{Fe} / \mathrm{H}] \leq+0.4$ and ages $<7$ Gyr)

2. A population of old clusters with a broad distribution of metallicities (metallicities $[\mathrm{Fe} / \mathrm{H}] \leq-0.3$ and ages $\geq 10 \mathrm{Gyr}$ )

3. A population of metal poor, intermediate age clusters (metallicities $[\mathrm{Fe} / \mathrm{H}] \leq-0.9$ and ages $<7$ Gyr)

While populations (1) and (2) are well known in the literature (e.g., Peng et al. 2004b), the third population of metal poor and intermediate age clusters has never been described in the literature.

If this result can be reproduced by independent analyses of datasets more rich or of higher quality, respectively, e.g. by NIR and/or high quality spectral observations for a cluster sample as large as the sample analysed here, hopefully being available in the future, numerous implications for the merger history of NGC 5128 are to be expected.

E.g. could this metal-poor, intermediate age population of GCs have formed in a starburst during the accretion of a gas-rich low luminosity, and hence low metallicity companion at some time $<7$ Gyr ago. A correspondingly younger counterpart to the Magellanic Clouds could have been a suitable candidate. 

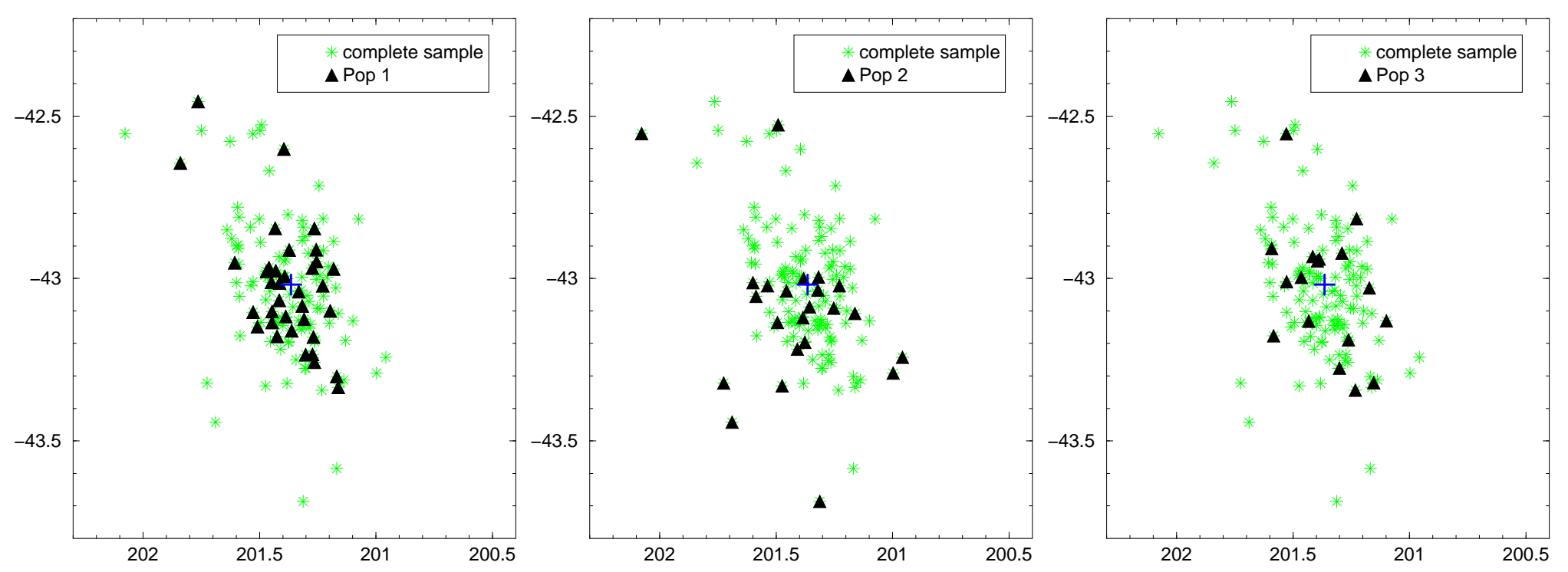

Fig. 5.5: Positions of all cluster for the three populations defined in the text (black triangles): Population 1 (left panel, population 2 (middle panel), and population 3 (right panel). To be compared with Fig. 5.1, the complete sample of clusters analysed is plotted as well (green stars). 


\subsubsection{Correlations with galactic position}

To check if the subpopulations we distinguish correlate with peculiar spacial distributions, we show in Fig. 5.5 the positions of all three populations.

The sample of metal-rich clusters with young to intermediate ages (population 1) are centrally concentrated with only three outlyers in the upper part. Compared with that, the sample of old clusters exhibiting a broad distribution of metallicities (population 2) are spread over the whole galaxy, less concentrated towards the galactic center, in agreement with results obtained by Harris et al. (2004) and in analogy to what is found in general for the blue and red GC populations in elliptical and S0 galaxies.

Surprisingly, the peculiar population 3, consisting of metal poor, intermediate age clusters, have only very few clusters close to the center. However, its distribution seems to be very much restricted to the central part of NGC 5128, similar to population 1 . No clusters from this population 3 are found in outer parts of the galaxy.

\subsection{Summary}

I have analysed an unprecedented large sample of both UBVRI broad-band SEDs and spectral indices for the GCS of the large elliptical galaxy NGC 5128.

Despite the relatively poor quality of the data (wavelength basis too small for independent constrains of ages and, hence, metallicities using the photometric dataset; Lick indices determined on low $\mathrm{S} / \mathrm{N}$ spectra) which do not allow to constrain ages and metallicities with reasonable precision by analysing each dataset alone, a combined analysis using the analysis tool PRODUCT developed within the framework of this thesis allows independent determinations of ages and metallicities for a large fraction of the analysed sample with unprecedented precision.

I found bimodal metallicity and age distributions, with lowest/highest metallicity bins, however, probably being artefacts.

In addition to the cluster populations well known in the literature (old GCs from low to high metallicities, and young metal-rich GCs), I found a population of intermediate age, metal-poor GCs in NGC 5128 which has not been described in the literature before.

The origin of this population, which seems to be correlated with the galactic halo, could be a starburst during the accretion of a low metallicity companion at some time $<7$ Gyr ago.

\subsection{References}

Cardelli J.A., Clayton G.C., and Mathis J.S., 1989, ApJ 345, 245

Fritze-v. Alvensleben U., 2004, A\&A 414, 515

Gebhardt K., Kissler-Patig M., 1999, AJ 118, 1526

Harris G.L.H., Harris W.E., and Geisler D., 2004, AJ 128, 723

Held E.V., Federici L., Cacciari C., and Testa V., 2002, IAUS 207, 269 
Israel F.P., 1998, A\&A Rev. 8, 237

Kundu A., Whitmore B.C., 2001a, AJ 121, 2950

Kundu A., Whitmore B.C., 2001b, AJ 122, 1251

Peng E.W., Ford H.C., and Freeman K.C., 2004a, ApJS 150, 367

Peng E.W., Ford H.C., and Freeman K.C., 2004b, ApJ 602, 705

Peng E.W., 2005, private comunication

Rejkuba M., 2001, A\&A 369, 812

Schlegel D.J., Finkbeiner D.P., and Davis M., 1998, ApJ 500, 525 


\section{Chapter 6}

\section{Summary}

This thesis deals with a question fundamental for the exploration of galaxies with the question about the evolutionary histories of galaxies and how they can best be recovered.

In the first chapter, results are presented from a study focusing on the question to what precision and how far back in time star formation histories can be determined using integrated colours, Lick indices, or spectra, compared to CMDs. In this methodological study I compared different kinds of simplified SFHs, i.e. epochs of constant low and high SFRs and investigated how much can be revealed by integrated light data about the SFH of a galaxy.

As a main result, I conclude that SFH details can be recovered with similar accuracy from broad-band colours or SEDs, from Lick indices, and from low/intermediate resolution spectroscopy, with lookback times to distinguish between different scenarious of SF not longer than 1 to 4 Gyr.

In the same chapter, I presented a methodological application to a star field in the bar of the LMC for which both an integrated-light spectrum and a CMD is available. Agreement with the observed spectrum could be reached with a very simple three phase toy model. The agreement is comparable to the agreement reached with a much more complicated $\mathrm{SFH}$ derived from the CMD.

From these studies, I conclude that both from CMDs and integrated light (multi-band photometry as well as spectroscopy), SFRs during the last Gyr are very precisely recovered, SFRs between 1 and 3 Gyr ago are roughly recovered, and SFRs longer than 3 to 5 Gyr ago are only vaguely recovered. However, integrated light is more sensitive to the latest 1 Gyr of SF, CMDs are more sensitive to intermediate ages.

In the following two chapters I presented models incorporated into the evolutionary synthesis code GALEV and a set of new tools for the analysis of globular cluster systems in galaxies:

To cope with the observational progress that makes star cluster and globular cluster spectra accessible in a wide variety of external galaxies, I have computed a large grid of evolutionary synthesis models for simple stellar populations, including $25 \mathrm{Lick} / \mathrm{IDS}$ indices using the empirical calibrations of Worthey et 
al. (1994) and Worthey \& Ottaviani (1997). Comparison of the models with Galactic GC observations shows good agreement between models and data.

I find that the well-known and widely used age-sensitive indices $\mathrm{H} \delta_{A}$ and $\mathrm{H} \gamma_{A}$ also show a strong metallicity dependence.

I present a new advanced tool for interpreting absorption-line indices, the Lick index analysis tool. Following an $\chi^{2}$-approach, this tool determines age and metallicity, including their respective $\pm 1 \sigma$ uncertainties, using all, or any subset of, measured indices. Testing the tool against index measurements from various authors for Galactic GCs, which have reliable age and metallicity determinations from CMD analyses in the literature, yields very good agreement.

Index measurements for M31 clusters are analysed and compared to results from the literature, and a good agreement between my results and age and metallicity determinations from the literature is found. I show that the drawback of not having non-solar abundance ratio models does not seriously affect the results.

I have developed a new method for the combined analysis of broad-band SEDs and spectral indices, PRODUCT, which successfully allow to constrain ages and metallicities of individual GCs even in cases when poor datasets are available only.

I have tested my new method using photometry and Lick indices for star clusters in the LMC, and in the elliptical galaxy NGC 5128, and find that age and metallicity determinations obtained by analysing Lick indices can substantially be improved if a set of colours covering a wavelength basis as long as possible, at best from $\mathrm{U}$ through $\mathrm{K}$, is available, even if only very few spectral indices are available.

I further point at the important fact that colour-metallicity relations widely used in the literature for determining star clusters metallicities are valid only for old GCs, and can by no means be applied to GCSs which contain subpopulations of intermediate age clusters.

In the last chapter, I have presented applications of the models and analysis tools developed within the framework of this thesis to the GCS of the large elliptical galaxy NGC 5128, using a dataset consisting of both broad-band photometry and Lick indices for an unprecedentedly large sample of GCs.

Despite the relatively poor quality of these data (wavelength basis of the photometric dataset too small for independent constraints on ages and, hence, metallicities; Lick indices determined on low $\mathrm{S} / \mathrm{N}$ spectra), which do not allow to constrain ages and metallicities with reasonable precision by analysing each dataset alone, a combined analysis using the analysis tool PRODUCT allows independent determinations of ages and metallicities for a large fraction of the analysed sample with unprecedented precision.

In addition to the cluster populations well known in the literature (old GCs from low to high metallicities, and young metal-rich GCs), I found a population of intermediate age, metal-poor GCs in NGC 5128 which has not been described in the literature before. 
The origin of this population, which seems to be correlated with the galactic halo, could be a starburst during the accretion of a low-metallicity companion at some time $<7$ Gyr ago. 



\section{Chapter 7}

\section{Acknowledgements}

First of all, I would like to thank my thesis advisors Prof. Dr. Uta Fritze - v. Alvensleben and Dr. Richard de Grijs. Without their constant support, teaching and discussions this thesis would not have been possible. I am especially grateful to Uta for her supervision and the exceptionally good atmosphere within the Galaxy Evolution Group here at the University of Göttingen (not to forget her famous "Schokobrötchenseminar"), and to Richard for his great hospitality and many fruitful working hours during my stayings in Cambridge and Sheffield.

In addition, I would like to thank a number of persons for many fruitful exchanges of ideas, their support, and a good time. While the total list would be too long, I would like to single out some special people.

In Göttingen, this includes the present and former members of the Galaxy Evolution Group, namely Thorsten Tepper García, Peter Anders, Markus Hartmann, Peter Weilbacher, Marie Aylin Tyra, Harald Braun, and Jens Bicker, among others. Many special thanks to Thorsten and Ralf for their invaluable support during the last days! A special "thank you" as well to Matthias Zetzl for his constant support with all software related questions.

Many thanks to Dr. Eric Peng for kindly providing me with his unpublished Lick index measurements for the GCS of NGC 5128, and to Prof. Dr. Guy Worthey for his kind advice during the implementation of his Lick index calibrations into the GALEV evolutionary synthesis code.

Last but not least, I am very grateful to Prof. Dr. Doug Geisler and Prof. Dr. Tom Richtler at the Universidad de Concepcion, Chile, for their hospitality and support during six great weeks in Chile.

Many thanks as well to uncountable astronomers all around the globe for making "scientific community" more than a myth but a reality I had the opportunity to experience on many international conferences and meetings. Thank you all for countless discussions and good times!

This work is partially supported by DFG grant Fr 916/11-1, Fr 916/11-2, and Fr 916/11-3. 



\section{Lebenslauf}

Name:

Geburtstag:

Geburtsort:

Staatsangehörigkeit:

Promotion:

Diplomarbeit:

Diplomstudium: (Physik)

Masterstudium: (Philosophie)

Schulbildung:
Thomas Lilly

21.08.1974

Bonn

Deutsch

seit $02 / 2003$

an der Georg-August-Universität Göttingen.

Betreuer: Prof. Dr. U. Fritze - v. Alvensleben (Göttingen, D; Hatfield, UK) und Dr. R. de Grijs (Sheffield, $\mathrm{UK})$.

Thema: Exploring the formation histories of galaxies globular clusters and beyond

angestrebtes Datum der Verteidigung: 12.07.2007

$02 / 2002$ bis $02 / 2003$

an der Georg-August-Universität Göttingen.

Betreuerin: Prof. Dr. Uta Fritze - v. Alvensleben (Göttingen, D; Hatfield, UK)

Thema: Analyse von Sternentstehungvergangenheiten in Galaxien - Ein methodischer Vergleich am Beispiel der $L M C$

Diplomprüfung: 7. Februar 2003

Notendurchschnitt $=1.25$

04/1999 - 01/2003

an der Georg-August-Universität G" ottingen.

10/1996 - 03/1999

an der Friedrich-Wilhelms-Universität Bonn.

Vordiplom 25. März 1999

Notendurchschnitt $=2.0$

04/1999 - 07/2003

an der Georg-August-Universität Göttingen.

(ohne Abschluss)

$1985-1994$

am Collegium Josephinum Bonn (Gymnasium)

Abitur im Juni 1994

Notendurchschnitt $=2.3$ 
